SUPPORTING INFROMATION

\title{
Intramolecular Imidoylative Heck Reaction: Synthesis of Cyclic Ketoimines from Functionalized Isocyanide
}

Jian Wang, Shi Tang and Qiang Zhu*

Contents




\section{General Information}

${ }^{1} \mathrm{H}$ NMR (400 MHz) and ${ }^{13} \mathrm{C}$ NMR $(125 \mathrm{MHz})$ were registered on $400 \mathrm{M}$ and $500 \mathrm{M}$ spectrometers, respectively. Chemical shifts were reported in units (ppm) by assigning TMS resonance in the ${ }^{1} \mathrm{H}$ spectrum as $0.00 \mathrm{ppm}, \mathrm{CDCl}_{3}$ resonance in the ${ }^{13} \mathrm{C}$ spectrum as $77.0 \mathrm{ppm}$. All coupling constants ( $J$ values) were reported in Hertz (Hz). NMR analysis was carried out at $298 \mathrm{~K}$ unless noted otherwise. HRMS was obtained on an ESI-LC-MS/MS spectrometer.

\section{Preparation of Starting Materials}

ethyl 2-isobutyl-2-isocyanohept-6-enoate (1a): ${ }^{1,2}$
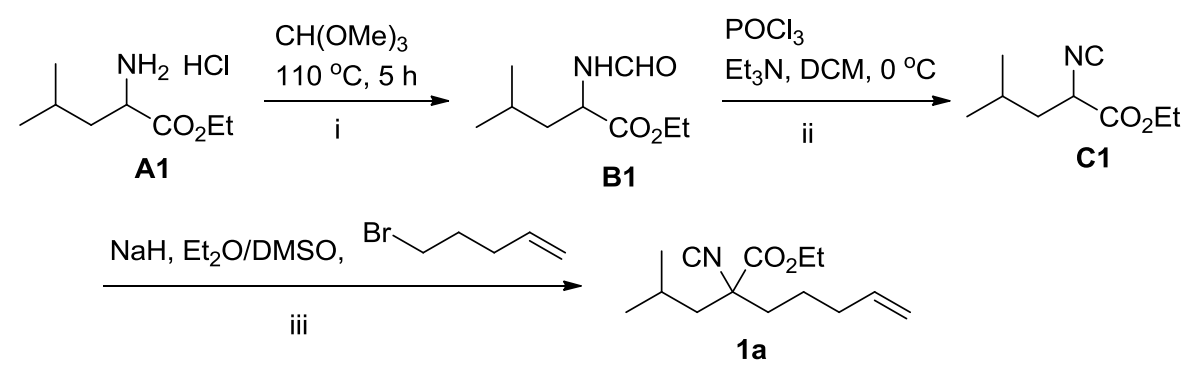

(i) A $250 \mathrm{~mL}$ round bottom flask charged with $100 \mathrm{~mL}$ of $\mathrm{CH}(\mathrm{OMe})_{3}$ (solvent) and A1 (10 mmol, $1.95 \mathrm{~g})$ was heated at $110{ }^{\circ} \mathrm{C}$ for $5 \mathrm{~h}$. The solvent was removed under reduced pressure to afford the crude product B1 for the next step without further purification.

(ii) To a solution of crude B1 (10 mmol, 1.0 equiv) and $\mathrm{Et}_{3} \mathrm{~N}$ (4 equiv) in $100 \mathrm{~mL}$ of dry $\mathrm{DCM}$ was added $\mathrm{POCl}_{3}\left(1.2\right.$ equiv) dropwise in $30 \mathrm{~min}$ at $-20{ }^{\circ} \mathrm{C}$. The reaction mixture was stirred for $4 \mathrm{~h}$. Then $10 \mathrm{~mL}$ of $\mathrm{H}_{2} \mathrm{O}$ was added dropwise to the reaction mixture carefully at $-20{ }^{\circ} \mathrm{C}$. The crude reaction mixture was extracted with DCM (100 $\mathrm{mL} \times 3)$ and washed with brine $(100 \mathrm{~mL})$. The organic phase was concentrated in vасио and the residue was purified by silica gel flash column chromatography (petroleum ether : EtOAc $=8: 1$ ) to afford the product $\mathbf{C 1}$ as colorless liquid in $85 \%$ yield.

(iii) An oven-dried $100 \mathrm{~mL}$ round bottom flask charged with a stir-bar and $\mathrm{NaH}$ (60\%) (10 mmol, 2 equiv) was vacuumed and refilled with Ar for 3 times. A mixture solution of DMSO $(2 \mathrm{~mL})$ and $\mathrm{Et}_{2} \mathrm{O}(50 \mathrm{~mL})$ was added to the flask using a syringe. Then $5 \mathrm{mmol}$ of $\mathbf{C 1}$ (1.0 equiv) was added to the mixture dropwise in $10 \mathrm{~min}$ at room temperature and the reaction mixture was stirred for $30 \mathrm{~min}$. Two equivalents of 
5-bromopent-1-ene was added with a syringe and the reaction mixture was stirred for $30 \mathrm{~min}$. Then $2 \mathrm{~mL}$ of $\mathrm{H}_{2} \mathrm{O}$ was added dropwise to the reaction mixture carefully at room temperature. The crude reaction mixture was extracted with DCM $(20 \mathrm{~mL} \times 3)$ and washed with brine $(20 \mathrm{~mL})$. The organic phase was concentrated in vacuo and the residue was purified by silica gel flash column chromatography (petroleum ether : EtOAc $=16: 1$ ) to afford the product $1 \mathbf{a}$ as colorless liquid in $91 \%$ yield. (new compound)

${ }^{1} \mathrm{H}$ NMR $\left(400 \mathrm{MHz}, \mathrm{CDCl}_{3}\right): \delta$ 5.78-5.68 (m, 1H), 5.02-4.95 (m, 2H), 4.25-4.20 (m, 2H), 2.07-2.01 (m, 2H), 1.92-1.81 (m, 3H), 1.75-1.65 (m, 3H), $1.30(\mathrm{t}, J=7.2 \mathrm{~Hz}$, $3 \mathrm{H}), 0.98(\mathrm{~d}, J=6.8 \mathrm{~Hz}, 3 \mathrm{H}), 0.84(\mathrm{~d}, J=6.8 \mathrm{~Hz}, 3 \mathrm{H}) ;{ }^{13} \mathrm{C} \mathrm{NMR}\left(125 \mathrm{MHz}, \mathrm{CDCl}_{3}\right)$ : $\delta 169.3,159.6,137.4,115.4,67.6,62.4,47.1,40.1,32.9,25.0,23.7,23.1,22.3,14.0$; HRMS: calcd for $\mathrm{C}_{14} \mathrm{H}_{24} \mathrm{NO}_{2}\left(\mathrm{M}^{+}+\mathrm{H}\right) 238.1802$; found 238.1804 .

methyl 2-isocyano-2-isopropylhept-6-enoate (1b):

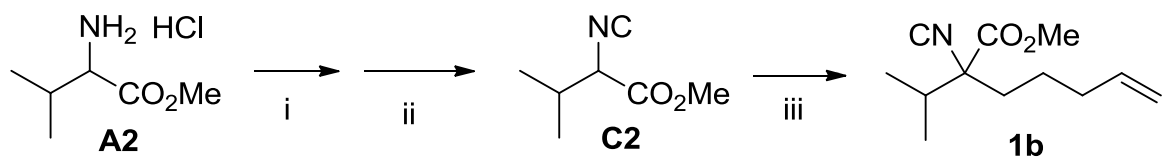

(i) A $250 \mathrm{~mL}$ round bottom flask charged with $50 \mathrm{~mL}$ of $\mathrm{CH}(\mathrm{OMe})_{3}$ (solvent) and A2 $(5 \mathrm{mmol}, 835 \mathrm{mg})$ was heated at $110{ }^{\circ} \mathrm{C}$ for $5 \mathrm{~h}$. The solvent was removed under reduced pressure to afford the crude product $\mathbf{B 2}$ for the next step without further purification.

(ii) To a solution of crude $\mathbf{B 2}$ ( $5 \mathrm{mmol}, 1.0$ equiv) and $\mathrm{Et}_{3} \mathrm{~N}$ (4 equiv) in $50 \mathrm{~mL}$ of dry DCM was added $\mathrm{POCl}_{3}\left(1.2\right.$ equiv) dropwise in $30 \mathrm{~min}$ at $-20{ }^{\circ} \mathrm{C}$. The reaction mixture was stirred for $4 \mathrm{~h}$. Then $10 \mathrm{~mL}$ of $\mathrm{H}_{2} \mathrm{O}$ was added dropwise to the reaction mixture carefully at $-20{ }^{\circ} \mathrm{C}$. The crude reaction mixture was extracted with DCM (100 $\mathrm{mL} \times 3)$ and washed with brine $(100 \mathrm{~mL})$. The organic phase was concentrated in vacuo and the residue was purified by silica gel flash column chromatography (petroleum ether : $\mathrm{EtOAc}=8: 1$ ) to afford the product $\mathbf{C 2}$ as colorless liquid in $78 \%$ yield.

(iii) An oven-dried $100 \mathrm{~mL}$ round bottom flask charged with a stir-bar and $\mathrm{NaH}(60 \%)$ (4 mmol, 2 equiv) was vacuumed and refilled with Ar for 3 times. A mixture solution of DMSO $(1 \mathrm{~mL})$ and $\mathrm{Et}_{2} \mathrm{O}(20 \mathrm{~mL})$ was added to the flask using a syringe. Then 2 mmol of $\mathbf{C 2}$ (1.0 equiv) was added to the mixture dropwise in $10 \mathrm{~min}$ at room temperature and the reaction mixture was stirred for $30 \mathrm{~min}$. Two equivalents of 
5-bromopent-1-ene was added with a syringe and the reaction mixture was stirred for $30 \mathrm{~min}$. Then $2 \mathrm{~mL}$ of $\mathrm{H}_{2} \mathrm{O}$ was added dropwise to the reaction mixture carefully at room temperature. The crude reaction mixture was extracted with DCM $(20 \mathrm{~mL} \times 3)$ and washed with brine $(20 \mathrm{~mL})$. The organic phase was concentrated in vacuo and the residue was purified by silica gel flash column chromatography (petroleum ether : EtOAc $=16: 1$ ) to afford the product $\mathbf{1 b}$ as colorless liquid in $87 \%$ yield. (new compound)

${ }^{1} \mathrm{H}$ NMR $\left(400 \mathrm{MHz}, \mathrm{CDCl}_{3}\right): \delta$ 5.74-5.66 (m, 1H), 5.00-4.92 (m, 2H), $3.76(\mathrm{~s}, 3 \mathrm{H})$, 2.13-2.01 (m, 3H), 1.81-1.77 (m, 2H), 1.66-1.59 (m, 1H), 1.23-1.16 (m, 1H), 1.01 (d, $J=6.8 \mathrm{~Hz}, 3 \mathrm{H}), 0.90(\mathrm{~d}, J=6.8 \mathrm{~Hz}, 3 \mathrm{H}) ;{ }^{13} \mathrm{C} \mathrm{NMR}\left(125 \mathrm{MHz}, \mathrm{CDCl}_{3}\right): \delta 169.5$, 159.5, 137.4, 115.4, 53.0, 36.6, 35.6, 33.0, 23.6, 17.8, 16.3; HRMS: calcd for $\mathrm{C}_{12} \mathrm{H}_{20} \mathrm{NO}_{2}\left(\mathrm{M}^{+}+\mathrm{H}\right)$ 210.1489; found 210.1490.

methyl 2-(sec-butyl)-2-isocyanohept-6-enoate (1c):

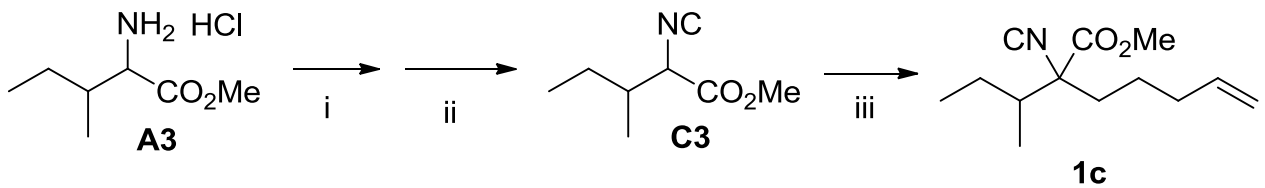

(i) A $250 \mathrm{~mL}$ round bottom flask charged with $50 \mathrm{~mL}$ of $\mathrm{CH}(\mathrm{OMe})_{3}$ (solvent) and A3 (5 mmol, $905 \mathrm{mg}$ ) was heated at $110{ }^{\circ} \mathrm{C}$ for $5 \mathrm{~h}$. The solvent was removed under reduced pressure to afford the crude product $\mathbf{B 3}$ for the next step without further purification.

(ii) To a solution of crude $\mathbf{B 3}$ ( $5 \mathrm{mmol}, 1.0$ equiv) and $\mathrm{Et}_{3} \mathrm{~N}$ (4 equiv) in $50 \mathrm{~mL}$ of dry DCM was added $\mathrm{POCl}_{3}\left(1.2\right.$ equiv) dropwise in $30 \mathrm{~min}$ at $-20{ }^{\circ} \mathrm{C}$. The reaction mixture was stirred for $4 \mathrm{~h}$. Then $10 \mathrm{~mL}$ of $\mathrm{H}_{2} \mathrm{O}$ was added dropwise to the reaction mixture carefully at $-20{ }^{\circ} \mathrm{C}$. The crude reaction mixture was extracted with DCM (100 $\mathrm{mL} \times 3)$ and washed with brine $(100 \mathrm{~mL})$. The organic phase was concentrated in vacuo and the residue was purified by silica gel flash column chromatography (petroleum ether : EtOAc $=8: 1$ ) to afford the product $\mathbf{C 3}$ as colorless liquid in $89 \%$ yield.

(iii) An oven-dried $100 \mathrm{~mL}$ round bottom flask charged with a stir-bar and $\mathrm{NaH}(60 \%)$ ( 4 mmol, 2 equiv) was vacuumed and refilled with Ar for 3 times. A mixture solution of DMSO $(1 \mathrm{~mL})$ and $\mathrm{Et}_{2} \mathrm{O}(20 \mathrm{~mL})$ was added to the flask using a syringe. Then 2 mmol of $\mathbf{C 3}$ (1.0 equiv) was added to the mixture dropwise in $10 \mathrm{~min}$ at room temperature and the reaction mixture was stirred for $30 \mathrm{~min}$. Two equivalents of 
5-bromopent-1-ene was added with a syringe and the reaction mixture was stirred for $30 \mathrm{~min}$. Then $2 \mathrm{~mL}$ of $\mathrm{H}_{2} \mathrm{O}$ was added dropwise to the reaction mixture carefully at room temperature. The crude reaction mixture was extracted with DCM $(20 \mathrm{~mL} \times 3)$ and washed with brine $(20 \mathrm{~mL})$. The organic phase was concentrated in vacuo and the residue was purified by silica gel flash column chromatography (petroleum ether : EtOAc $=16: 1$ ) to afford the product 1c as colorless liquid in $76 \%$ yield. (new compound)

${ }^{1} \mathrm{H}$ NMR $\left(400 \mathrm{MHz}, \mathrm{CDCl}_{3}\right): \delta$ 5.77-5.70 (m, 1H), 5.03-4.95 (m, 2H), $3.78(\mathrm{~s}, 3 \mathrm{H})$, 2.08-2.03 (m, 2H), 1.89-1.70 (m, 3H), 1.69-1.62 (m, 1H), 1.32-1.17 (m, 1H), 1.01 (d, $J=6.8 \mathrm{~Hz}, 2 \mathrm{H}), 0.96-0.88(\mathrm{~m}, 4 \mathrm{H}) ;{ }^{13} \mathrm{C} \mathrm{NMR}\left(125 \mathrm{MHz}, \mathrm{CDCl}_{3}\right): \delta 169.8,159.4$, 137.5, 115.4, 73.3, 53.0, 42.1, 36.8, 33.1, 25.0, 23.5, 12.5, 11.8; HRMS: calcd for $\mathrm{C}_{13} \mathrm{H}_{22} \mathrm{NO}_{2}\left(\mathrm{M}^{+}+\mathrm{H}\right)$ 224.1645; found 224.1649.

methyl 2-(tert-butyl)-2-isocyanohept-6-enoate (1d):
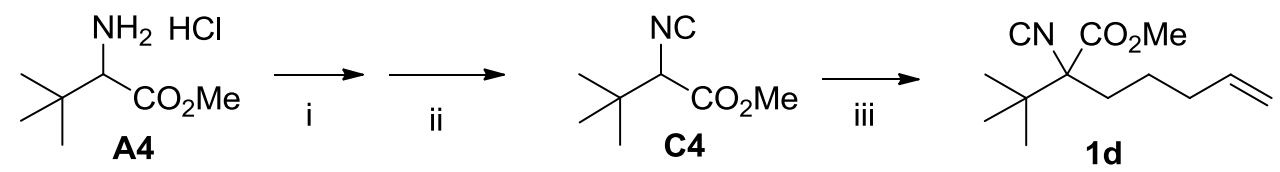

(i) A $250 \mathrm{~mL}$ round bottom flask charged with $50 \mathrm{~mL}$ of $\mathrm{CH}(\mathrm{OMe})_{3}$ (solvent) and A4 (5 mmol, $905 \mathrm{mg})$ was heated at $110{ }^{\circ} \mathrm{C}$ for $5 \mathrm{~h}$. The solvent was removed under reduced pressure to afford the crude product B4 for the next step without further purification.

(ii) To a solution of crude $\mathbf{B} 4$ ( $5 \mathrm{mmol}, 1.0$ equiv) and $\mathrm{Et}_{3} \mathrm{~N}$ (4 equiv) in $50 \mathrm{~mL}$ of dry DCM was added $\mathrm{POCl}_{3}\left(1.2\right.$ equiv) dropwise in $30 \mathrm{~min}$ at $-20{ }^{\circ} \mathrm{C}$. The reaction mixture was stirred for $4 \mathrm{~h}$. Then $10 \mathrm{~mL}$ of $\mathrm{H}_{2} \mathrm{O}$ was added dropwise to the reaction mixture carefully at $-20{ }^{\circ} \mathrm{C}$. The crude reaction mixture was extracted with DCM (100 $\mathrm{mL} \times 3)$ and washed with brine $(100 \mathrm{~mL})$. The organic phase was concentrated in vасио and the residue was purified by silica gel flash column chromatography (petroleum ether : EtOAc $=8: 1$ ) to afford the product $\mathbf{C 4}$ as colorless liquid in $65 \%$ yield.

(iii) An oven-dried $100 \mathrm{~mL}$ round bottom flask charged with a stir-bar and $\mathrm{NaH}$ (60\%) (4 mmol, 2 equiv) was vacuumed and refilled with Ar for 3 times. A mixture solution of DMSO $(1 \mathrm{~mL})$ and $\mathrm{Et}_{2} \mathrm{O}(20 \mathrm{~mL})$ was added to the flask using a syringe. Then $2 \mathrm{mmol}$ of $\mathbf{C 4}$ (1.0 equiv) was added to the mixture dropwise in $10 \mathrm{~min}$ at room temperature and the reaction mixture was stirred for $30 \mathrm{~min}$. Two equivalents of 
5-bromopent-1-ene was added with a syringe and the reaction mixture was stirred for $30 \mathrm{~min}$. Then $2 \mathrm{~mL}$ of $\mathrm{H}_{2} \mathrm{O}$ was added dropwise to the reaction mixture carefully at room temperature. The crude reaction mixture was extracted with DCM $(20 \mathrm{~mL} \times 3)$ and washed with brine $(20 \mathrm{~mL})$. The organic phase was concentrated in vacuo and the residue was purified by silica gel flash column chromatography (petroleum ether : EtOAc $=16: 1$ ) to afford the product $1 \mathbf{d}$ as colorless liquid in $51 \%$ yield. (new compound)

${ }^{1} \mathrm{H}$ NMR $\left(400 \mathrm{MHz}, \mathrm{CDCl}_{3}\right): \delta$ 5.82-5.72 (m, 1H), 5.05-4.97 (m, 2H), $3.79(\mathrm{~s}, 3 \mathrm{H})$, 2.11-1.99 (m, 3H), 1.75-1.64 (m, 2H), 1.28-1.16 (m, 1H), 1.07 (s, 9H); ${ }^{13} \mathrm{C}$ NMR $(125$ $\left.\mathrm{MHz}, \mathrm{CDCl}_{3}\right): \delta 169.1,159.8,138.0,115.6,76.1,53.1,38.3,33.5,32.9,25.9,24.6$; HRMS: calcd for $\mathrm{C}_{13} \mathrm{H}_{22} \mathrm{NO}_{2}\left(\mathrm{M}^{+}+\mathrm{H}\right)$ 224.1645; found 224.1642.

\section{ethyl 2-isobutyl-2-isocyanohex-5-enoate (1e):}<smiles>CCOC(CC(C)C)CC(C)C</smiles>

C1

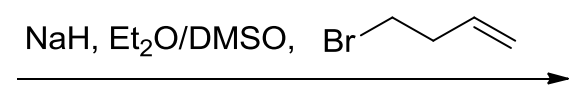

iii

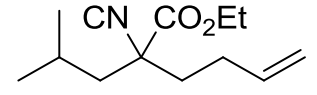

$1 e$

(iii) An oven-dried $100 \mathrm{~mL}$ round bottom flask charged with a stir-bar and $\mathrm{NaH}$ (60\%) (4 mmol, 2 equiv) was vacuumed and refilled with Ar for 3 times. A mixture solution of DMSO $(1 \mathrm{~mL})$ and $\mathrm{Et}_{2} \mathrm{O}(20 \mathrm{~mL})$ was added to the flask using a syringe. Then $2 \mathrm{mmol}$ of $\mathbf{C 1}$ (1.0 equiv) was added to the mixture dropwise in $10 \mathrm{~min}$ at room temperature and the reaction mixture was stirred for $30 \mathrm{~min}$. Two equivalents of 4-bromobut-1-ene was added with a syringe and the reaction mixture was stirred for 30 min. Then $2 \mathrm{~mL}$ of $\mathrm{H}_{2} \mathrm{O}$ was added dropwise to the reaction mixture carefully at room temperature. The crude reaction mixture was extracted with DCM $(20 \mathrm{~mL} \times 3)$ and washed with brine $(20 \mathrm{~mL})$. The organic phase was concentrated in vacuo and the residue was purified by silica gel flash column chromatography (petroleum ether : EtOAc $=16: 1$ ) to afford the product $1 \mathrm{e}$ as colorless liquid in $91 \%$ yield. (new compound)

${ }^{1} \mathrm{H}$ NMR (400 MHz, $\left.\mathrm{CDCl}_{3}\right): \delta$ 5.77-5.70 (m, 1H), 5.07-4.97 (m, 2H), 4.26-4.20 (m, $2 \mathrm{H}), 2.32-2.30(\mathrm{~m}, 1 \mathrm{H}), 2.02-1.90(\mathrm{~m}, 2 \mathrm{H}), 1.88-1.81(\mathrm{~m}, 3 \mathrm{H}), 1.75-1.67(\mathrm{~m}, 1 \mathrm{H})$, $1.31(\mathrm{t}, J=7.2 \mathrm{~Hz}, 3 \mathrm{H}), 1.00(\mathrm{~d}, J=6.4 \mathrm{~Hz}, 3 \mathrm{H}), 0.86(\mathrm{~d}, J=6.4 \mathrm{~Hz}, 3 \mathrm{H}) ;{ }^{13} \mathrm{C} \mathrm{NMR}$ $\left(125 \mathrm{MHz}, \mathrm{CDCl}_{3}\right): \delta 169.1,159.8,136.1,116.0,67.3,62.5,47.3,39.9,28.2,25.0$, 23.7, 22.3, 14.0; HRMS: calcd for $\mathrm{C}_{13} \mathrm{H}_{22} \mathrm{NO}_{2}\left(\mathrm{M}^{+}+\mathrm{H}\right)$ 224.1645; found 224.1647. 
(E)-ethyl 2-isobutyl-2-isocyano-5-phenylpent-4-enoate (1f):<smiles>CCOC(=O)C(C)CC(C)C</smiles>

C1

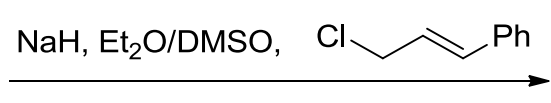

iii

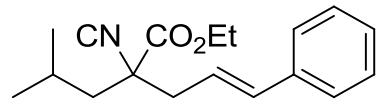

$1 f$

(iii) An oven-dried $100 \mathrm{~mL}$ round bottom flask charged with a stir-bar and $\mathrm{NaH}$ (60\%) (4 mmol, 2 equiv) was vacuumed and refilled with Ar for 3 times. A mixture solution of DMSO $(1 \mathrm{~mL})$ and $\mathrm{Et}_{2} \mathrm{O}(20 \mathrm{~mL})$ was added to the flask using a syringe. Then $2 \mathrm{mmol}$ of $\mathbf{C 1}$ (1.0 equiv) was added to the mixture dropwise in $10 \mathrm{~min}$ at room temperature and the reaction mixture was stirred for $30 \mathrm{~min}$. Two equivalents of (E)-(3-chloroprop-1-en-1-yl)benzene was added with a syringe and the reaction mixture was stirred for $30 \mathrm{~min}$. Then $2 \mathrm{~mL}$ of $\mathrm{H}_{2} \mathrm{O}$ was added dropwise to the reaction mixture carefully at room temperature. The crude reaction mixture was extracted with DCM $(20 \mathrm{~mL} \times 3)$ and washed with brine $(20 \mathrm{~mL})$. The organic phase was concentrated in vacuo and the residue was purified by silica gel flash column chromatography (petroleum ether : EtOAc $=16: 1$ ) to afford the product $\mathbf{1 f}$ as yellow oil in $57 \%$ yield. (new compound)

${ }^{1} \mathrm{H}$ NMR (400 MHz, $\left.\mathrm{CDCl}_{3}\right): \delta 7.37-7.23(\mathrm{~m}, 5 \mathrm{H}), 6.51(\mathrm{~d}, J=16.0 \mathrm{~Hz}, 1 \mathrm{H})$, 6.21-6.13 (m, 1H), 4.23 (q, $J=6.8 \mathrm{~Hz}, 2 \mathrm{H}), 2.80-2.64(\mathrm{~m}, 2 \mathrm{H}), 1.97-1.89(\mathrm{~m}, 2 \mathrm{H})$, 1.83-1.79 (m, 1H), 1.28 (t, $J=6.8 \mathrm{~Hz}, 3 \mathrm{H}), 1.02(\mathrm{~d}, \mathrm{~J}=6.0 \mathrm{~Hz}, 3 \mathrm{H}), 0.89$ (d, $J=6.0$ $\mathrm{Hz}, 3 \mathrm{H}) ;{ }^{13} \mathrm{C} \mathrm{NMR}\left(125 \mathrm{MHz}, \mathrm{CDCl}_{3}\right): \delta 169.2,160.4,136.9,136.0,128.9,128.2$, $126.8,121.5,67.9,62.9,46.8,44.6,25.4,24.0,22.6,14.4$; HRMS: calcd for $\mathrm{C}_{18} \mathrm{H}_{24} \mathrm{NO}_{2}\left(\mathrm{M}^{+}+\mathrm{H}\right) 286.1802$; found 286.1798.

ethyl 2-isocyano-2-(pent-4-en-1-yl)hept-6-enoate (1g):

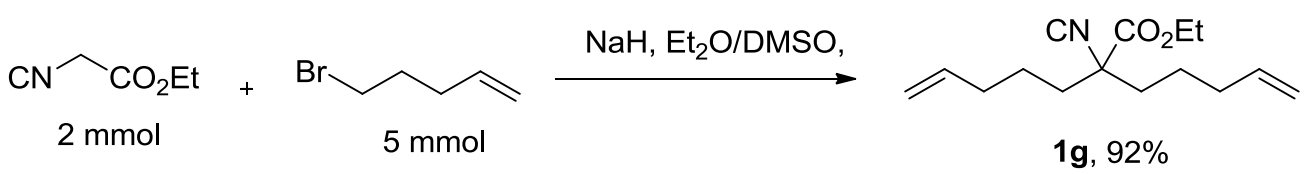

(iii) An oven-dried $100 \mathrm{~mL}$ round bottom flask charged with a stir-bar and $\mathrm{NaH}$ (60\%) (6 mmol, 3 equiv) was vacuumed and refilled with Ar for 3 times. A mixture solution of DMSO $(1 \mathrm{~mL})$ and $\mathrm{Et}_{2} \mathrm{O}(20 \mathrm{~mL})$ was added to the flask using a syringe. Then 2 mmol of ethyl 2-isocyanoacetate (1.0 equiv) was added to the mixture dropwise in $10 \mathrm{~min}$ at room temperature and the reaction mixture was stirred for 30 min. Three equivalents of 5-bromopent-1-ene was added with a syringe and the reaction mixture was stirred for $30 \mathrm{~min}$. Then $2 \mathrm{~mL}$ of $\mathrm{H}_{2} \mathrm{O}$ was added dropwise to the 
reaction mixture carefully at room temperature. The crude reaction mixture was extracted with DCM $(20 \mathrm{~mL} \times 3)$ and washed with brine $(20 \mathrm{~mL})$. The organic phase was concentrated in vacuo and the residue was purified by silica gel flash column chromatography (petroleum ether : EtOAc $=16: 1$ ) to afford the product $1 \mathrm{~g}$ as colorless liquid in $92 \%$ yield. (new compound)

${ }^{1} \mathrm{H}$ NMR $\left(400 \mathrm{MHz}, \mathrm{CDCl}_{3}\right): \delta$ 5.77-5.67 (m, 2H), 5.01-4.94 (m, 4H), 4.23 (q, J= 7.2 $\mathrm{Hz}, 2 \mathrm{H}), 2.07-2.02$ (m, 4H), 1.92-1.85 (m, 2H), 1.78-1.59 (m, 4H), 1.37-1.27 (m, 5H); ${ }^{13} \mathrm{C}$ NMR $\left(125 \mathrm{MHz}, \mathrm{CDCl}_{3}\right): \delta 168.8,159.1,137.4,115.4,68.3,62.5,38.5,33.0$, 23.2, 14.1; HRMS: calcd for $\mathrm{C}_{15} \mathrm{H}_{24} \mathrm{NO}_{2}\left(\mathrm{M}^{+}+\mathrm{H}\right)$ 250.1802; found 250.1802.

diethyl (6-isocyanoundeca-1,10-dien-6-yl)phosphonate (1h)

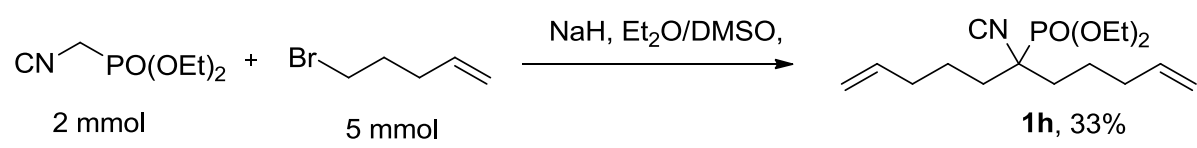

(iii) An oven-dried $100 \mathrm{~mL}$ round bottom flask charged with a stir-bar and $\mathrm{NaH}$ (60\%) (6 mmol, 3 equiv) was vacuumed and refilled with Ar for 3 times. A mixture solution of DMSO $(1 \mathrm{~mL})$ and $\mathrm{Et}_{2} \mathrm{O}(20 \mathrm{~mL})$ was added to the flask using a syringe. Then 2 mmol of diethyl (isocyanomethyl)phosphonate (1.0 equiv) was added to the mixture dropwise in $10 \mathrm{~min}$ at room temperature and the reaction mixture was stirred for $30 \mathrm{~min}$. Three equivalents of 5-bromopent-1-ene was added with a syringe and the reaction mixture was stirred for $30 \mathrm{~min}$. Then $2 \mathrm{~mL}$ of $\mathrm{H}_{2} \mathrm{O}$ was added dropwise to the reaction mixture carefully at room temperature. The crude reaction mixture was extracted with DCM $(20 \mathrm{~mL} \times 3)$ and washed with brine $(20 \mathrm{~mL})$. The organic phase was concentrated in vacuo and the residue was purified by silica gel flash column chromatography (petroleum ether : EtOAc $=8: 1$ ) to afford the product $\mathbf{1 h}$ as colorless liquid in $33 \%$ yield. (new compound)

${ }^{1} \mathrm{H}$ NMR (400 MHz, $\mathrm{CDCl}_{3}$ ): $\delta$ 5.82-5.72 (m, 2H), 5.05-4.97 (m, 4H), 4.27-4.19 (m, 4H), 2.10-2.05 (m, 4H), 1.93-1.75 (m, 4H), 1.65-1.54 (m, 4H), $1.36(\mathrm{t}, J=6.8 \mathrm{~Hz}$, $6 \mathrm{H}) ;{ }^{13} \mathrm{C}$ NMR $\left(125 \mathrm{MHz}, \mathrm{CDCl}_{3}\right): \delta 159.6,138.0,115.8,64.4(\mathrm{~d}, J=7.4 \mathrm{~Hz}), 62.0(\mathrm{~d}$, $J=155.4 \mathrm{~Hz}), 34.6,33.7,23.1(\mathrm{~d}, J=5.4 \mathrm{~Hz}), 16.8(\mathrm{~d}, \mathrm{~J}=5.6 \mathrm{~Hz})$; HRMS: calcd for $\mathrm{C}_{16} \mathrm{H}_{29} \mathrm{NO}_{3} \mathrm{P}\left(\mathrm{M}^{+}+\mathrm{H}\right)$ 314.1880; found 314.1885.

ethyl 2-isobutyl-2-isocyanopent-4-enoate (1i): 


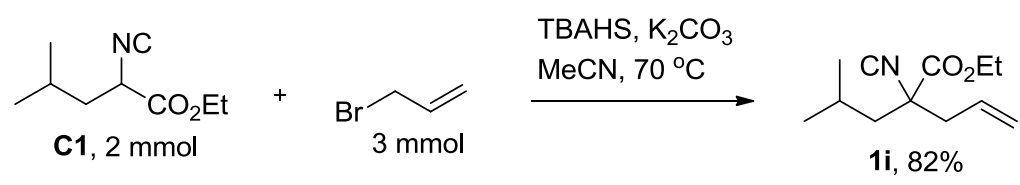

Compound 1i was prepared according to similar route of literature reported methods ${ }^{3}$ : To a solution of $\mathbf{C 1}(2 \mathrm{mmol})$ in acetonitrile $(20 \mathrm{~mL})$ were added $\mathrm{K}_{2} \mathrm{CO}_{3}(8$ mmol), TBAHS $(0.2 \mathrm{mmol})$, and 3-bromoprop-1-ene $(3 \mathrm{mmol})$. The mixture was heated at $70{ }^{\circ} \mathrm{C}$ until the reaction was completed, monitoring with TLC. Then the mixture was cooled and the solvent was removed and reduced pressure. $\mathrm{H}_{2} \mathrm{O}(10 \mathrm{~mL})$ and ethyl acetate $(20 \mathrm{~mL})$ were added into the mixture. The organic phase was seperated and the aqueous phase was extacted with ethyl acetate $(20.0 \mathrm{~mL} \times 3)$. The combined organic phase was concentrated in vacuo and the residue was purified by silica gel flash column chromatography (petroleum ether : EtOAc $=8: 1$ ) to afford $\mathbf{1 i}$ as colorless liquid in $82 \%$ yield. (new compound)

${ }^{1} \mathrm{H}$ NMR (400 MHz, $\mathrm{CDCl}_{3}$ ): $\delta$ 5.84-5.74 (m, 1H), 5.24-5.17 (m, 2H), 4.27-4.20 (m, 2H), 2.65-2.47 (m, 2H), 1.94-1.72 (m, 3H), $1.31(\mathrm{t}, J=7.2 \mathrm{~Hz}, 3 \mathrm{H}), 1.01(\mathrm{~d}, J=6.4$ $\mathrm{Hz}, 3 \mathrm{H}), 0.87(\mathrm{~d}, J=6.4 \mathrm{~Hz}, 3 \mathrm{H}) ;{ }^{13} \mathrm{C} \mathrm{NMR}\left(125 \mathrm{MHz}, \mathrm{CDCl}_{3}\right): \delta 168.9,159.8,130.0$, 120.9, 67.3, 62.5, 46.5, 44.9, 25.0, 23.6, 22.3, 14.1; HRMS: calcd for $\mathrm{C}_{12} \mathrm{H}_{20} \mathrm{NO}_{2}$ $\left(\mathrm{M}^{+}+\mathrm{H}\right)$ 210.1489; found 210.1485.

ethyl 2-isocyanopent-4-enoate $(\mathbf{1 j})$ :

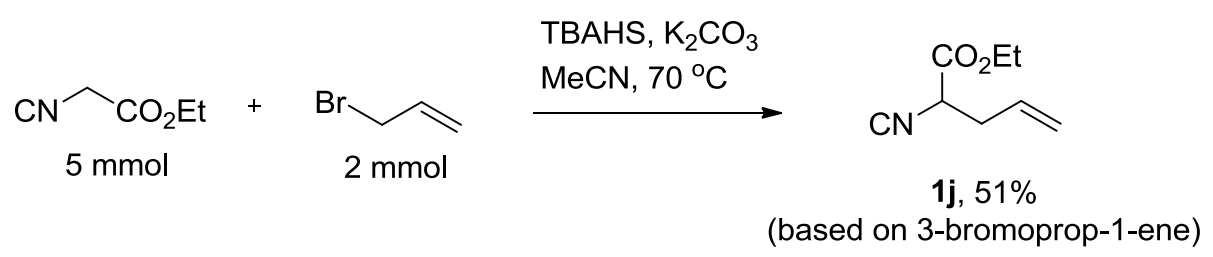

To a solution of ethyl 2-isocyanoacetate ( $5 \mathrm{mmol}, 2.5$ equiv) in acetonitrile (20 $\mathrm{mL})$ were added $\mathrm{K}_{2} \mathrm{CO}_{3}(10 \mathrm{mmol})$, TBAHS (0.2 mmol), and 3-bromoprop-1-ene (2 mmol). The mixture was heated at $70{ }^{\circ} \mathrm{C}$ until the reaction was completed, monitoring with TLC. Then the mixture was cooled and the solvent was removed and reduced pressure. $\mathrm{H}_{2} \mathrm{O}(10 \mathrm{~mL})$ and ethyl acetate $(20 \mathrm{~mL})$ were added into the mixture. The organic phase was seperated and the aqueous phase was extacted with ethyl acetate $(20.0 \mathrm{~mL} \times 3)$. The combined organic phase was concentrated in vacuo and the residue was purified by silica gel flash column chromatography (petroleum ether : EtOAc $=8: 1)$ to afford $\mathbf{1 i}$ as colorless liquid in $51 \%$ yield. (known compound) ${ }^{4}$

${ }^{1} \mathrm{H}$ NMR $\left(400 \mathrm{MHz}, \mathrm{CDCl}_{3}\right): \delta$ 5.81-5.71 (m, 1H), 5.24-5.19 (m, 2H), 4.30-4.20 (m, $3 \mathrm{H}), 2.69-2.55(\mathrm{~m}, 2 \mathrm{H}), 1.27(\mathrm{t}, J=7.2 \mathrm{~Hz}, 3 \mathrm{H}) ;{ }^{13} \mathrm{C} \mathrm{NMR}\left(125 \mathrm{MHz}, \mathrm{CDCl}_{3}\right): \delta$ 
166.1, 160.4, 130.4, 120.4, 62.7, 56.3, 37.0, 14.0; HRMS: calcd for $\mathrm{C}_{8} \mathrm{H}_{12} \mathrm{NO}_{2}\left(\mathrm{M}^{+}+\mathrm{H}\right)$ 154.0863; found 154.0861.

\section{Imidoylative Heck Reaction}

Table 1. Detailed Information of Conditions Optimization ${ }^{a}$

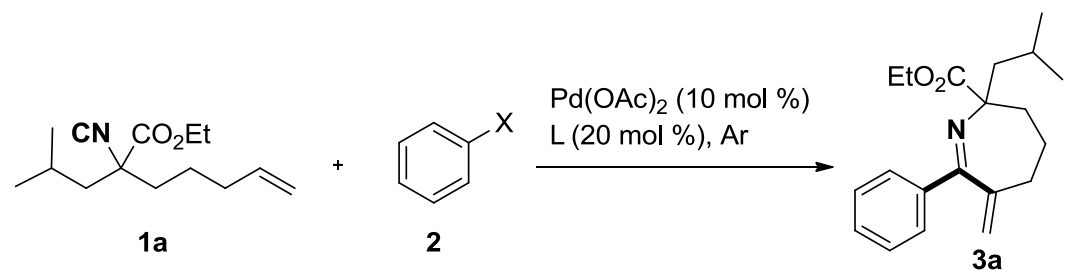

\begin{tabular}{|c|c|c|c|c|c|}
\hline entry & solvent & base (1.2 equiv) & ligand (20 mol \%) & temp $\left({ }^{\circ} \mathrm{C}\right)$ & yield $(\%)^{b}$ \\
\hline 1 & toluene & $\mathrm{Na}_{2} \mathrm{CO}_{3}$ & $\mathrm{PPh}_{3}$ & 80 & nd \\
\hline $2^{c}$ & toluene & $\mathrm{Na}_{2} \mathrm{CO}_{3}$ & $\mathrm{PPh}_{3}$ & 80 & 18 \\
\hline $3^{c}$ & toluene & $\mathrm{K}_{2} \mathrm{CO}_{3}$ & $\mathrm{PPh}_{3}$ & 80 & 18 \\
\hline $4^{c}$ & toluene & $\mathrm{Cs}_{2} \mathrm{CO}_{3}$ & $\mathrm{PPh}_{3}$ & 80 & 30 \\
\hline $5^{c}$ & toluene & CsOPiv & $\mathrm{PPh}_{3}$ & 80 & 70 \\
\hline $6^{c}$ & toluene & $\mathrm{Et}_{3} \mathrm{~N}$ & $\mathrm{PPh}_{3}$ & 80 & 25 \\
\hline $7^{c}$ & DMSO & CsOPiv & $\mathrm{PPh}_{3}$ & 80 & 70 \\
\hline $8^{c}$ & dioxane & CsOPiv & $\mathrm{PPh}_{3}$ & 80 & 33 \\
\hline $9^{c}$ & $\mathrm{CH}_{3} \mathrm{CN}$ & CsOPiv & $\mathrm{PPh}_{3}$ & 80 & 40 \\
\hline $10^{c, e}$ & toluene & CsOPiv & $\mathrm{PPh}_{3}$ & 80 & $81(79)^{f}$ \\
\hline $11^{c, e}$ & toluene & CsOPiv & dppb (10 mol \%) & 80 & 75 \\
\hline $12^{c, e}$ & toluene & CsOPiv & BINAP (10 mol \%) & 80 & trace \\
\hline $13^{c, e, g}$ & toluene & CsOPiv & $\mathrm{PPh}_{3}$ & 80 & 78 \\
\hline $14^{c, e, h}$ & toluene & CsOPiv (1.0 equiv) & $\mathrm{PPh}_{3}$ & 80 & 70 \\
\hline $15^{c, e, i}$ & toluene & CsOPiv & $\mathrm{PPh}_{3}$ & 80 & 85 (change) \\
\hline $16^{c, j}$ & toluene & CsOPiv & $\mathrm{PPh}_{3}$ & 80 & $56^{f}$ \\
\hline $17^{c, e}$ & toluene & CsOPiv & $\mathrm{PPh}_{3}$ & 70 & $45^{f}$ \\
\hline $18^{c, e, k}$ & toluene & CsOPiv & $\mathrm{PPh}_{3}$ & 70 & 0 \\
\hline $19^{d, e}$ & toluene & CsOPiv & $\mathrm{PPh}_{3}$ & 100 & $85(82)^{f}$ \\
\hline
\end{tabular}


${ }^{a}$ Reaction conditions: 1a $(0.2 \mathrm{mmol}), 2(0.30 \mathrm{mmol}), \mathrm{Pd}(\mathrm{OAc})_{2}(0.02 \mathrm{mmol}, 10 \mathrm{~mol} \%), \mathrm{PPh}_{3}(0.04 \mathrm{mmol}, 20$ mol \%), base $(0.24 \mathrm{mmol})$, Ar. A solution of 1a in solvent $(1 \mathrm{~mL})$ was added slowly within $1 \mathrm{~h} .{ }^{b} \mathrm{NMR}$ yield with 1-iodo-4-methoxybenzene as an internal standard. ${ }^{c} \mathrm{X}=\mathrm{I} .{ }^{d} \mathrm{X}=\mathrm{Br} .{ }^{e} \mathrm{~A}$ solution of $\mathbf{1 a}$ in toluene $(1 \mathrm{~mL})$ was added via a syringe pump within $1 \mathrm{~h} .{ }^{f}$ Isolated yield. ${ }^{g}$ One equivalent of $\mathrm{H} 2 \mathrm{O}$ was added in the reaction. ${ }^{h}$ One equivalent

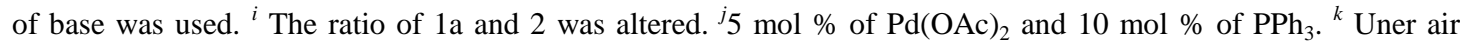
atmosphere.

The reaction conditions were screened with ethyl 2-isobutyl-2-isocyanohept-6-enoate $\mathbf{1 a}$ and iodobenzene as model substrates. However, a reaction of premixed reactants under normal Heck conditions $\left(\mathrm{Pd}(\mathrm{OAc})_{2}\right.$, $\mathrm{PPh}_{3}, \mathrm{Na}_{2} \mathrm{CO}_{3}$ in toluene) yielded only a messy mixture (entry 1 , Table 1 ). When a solution of isocyanide 1a in toluene was introduced to the reaction mixture slowly, the desired product, ethyl 2-isobutyl-6-methylene-7-phenyl-3,4,5,6-tetrahydro- $2 \mathrm{H}$-azepine-2-carboxylate $\quad 3 a$, was formed in 18\% NMR yield (entry 2, Table 1). Screening of bases revealed that CsOPiv was the base of choice for this imidoylative Heck reaction, and 3a could be obtained in $70 \%$ yield (entries 3-6). The reaction was equally efficient in DMSO, but less efficient in dioxane or $\mathrm{CH}_{3} \mathrm{CN}$ (entries 7-9). The yield of 3a was increased to $81 \%$ when a solution of $\mathbf{1 a}$ in $1 \mathrm{~mL}$ of toluene was added via a syringe pump during $1 \mathrm{~h}$ (entry 10). Dppb was also an efficient ligand (entry 11). Other phosphine ligands such as BINAP were tested as well, but resulted in trace product (entry 12). Considering that $\mathrm{PPh}_{3}$ was an easily available and inexpensive ligand, it was chosen in the reaction. When 1.0 equivalent of $\mathrm{H}_{2} \mathrm{O}$ was added in the reaction, 78\% NMR yield of product was generated, indicating that the reaction was well tolerated in moisture (entry 13). When using 1.0 equivalent of base, the yield was decreased to $70 \%$ (entry 14). If the ratio between the isocyanide and the aryl iodide are altered, the yield was similar (entry 15). Considering that aryl halides are less inexpensive than isocyanide, we used it in excess amount. The yield decreased to $45 \%$ at $70{ }^{\circ} \mathrm{C}$ (entry 16) and $56 \%$ with 5 mol \% of $\mathrm{Pd}(\mathrm{OAc})_{2}$ and $10 \mathrm{~mol} \%$ of $\mathrm{PPh}_{3}$ (entry 17). No product was generated under air atmosphere (entry 18). When using bromobenzene as the arylating reagent, the same product 3a was generated in $85 \%$ yield at elevated temperature $\left(100{ }^{\circ} \mathrm{C}\right.$, entry 19).

\section{General procedure:}



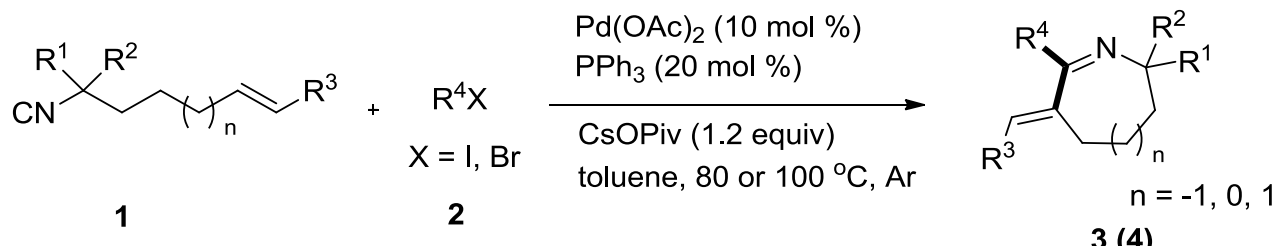

3 (4)

An oven-dried $25 \mathrm{~mL}$ Schlenk tube charged with $\mathrm{Pd}(\mathrm{OAc})_{2}(0.02 \mathrm{mmol}, 4.5 \mathrm{mg})$, $\mathrm{PPh}_{3}(0.04 \mathrm{mmol}, 10.5 \mathrm{mg})$ and CsOPiv $(0.24 \mathrm{mmol}, 56.0 \mathrm{mg})$ was vacuumed and refilled with $\mathrm{Ar}$ for 3 times. Then a solution of $2(0.3 \mathrm{mmol}, 38.4 \mu \mathrm{L})$ in $1.0 \mathrm{~mL}$ of toluene was added via a syringe and the tube was placed in an oil-bath at $80{ }^{\circ} \mathrm{C}$ for aryl iodide or $100{ }^{\circ} \mathrm{C}$ for aryl bromide. A solution of $\mathbf{1}(0.2 \mathrm{mmol})$ in $1.0 \mathrm{~mL}$ of toluene was introduced with a syringe pump to the reaction mixture during $1 \mathrm{~h}$. The crude reaction mixture was extracted with $\mathrm{DCM}(20 \mathrm{~mL} \times 3)$ and washed with brine $(20 \mathrm{~mL})$. The organic phase was concentrated in vacuo and the residue was purified by silica gel flash column chromatography to afford the corresponding cyclic imines.

\section{Characterization Data}<smiles>C=C1CCCC(COCC)(CC(C)C)N=C1c1ccccc1</smiles>

ethyl 2-isobutyl-6-methylene-7-phenyl-3,4,5,6-tetrahydro-2 $H$-azepine-2-carboxylate (3a)

Prepared from ethyl 2-isobutyl-2-isocyanohept-6-enoate $(47.4 \mathrm{mg}, 0.2 \mathrm{mmol}, 1.0$ equiv) and iodobenzene (61 $\mathrm{mg}, 0.3 \mathrm{mmol}, 1.5$ equiv) according to the general procedure. Column chromatography purification (EtOAc : petroleum ether $30: 1$ ) furnished the product $\mathbf{3 a}$ as colorless oil $(50 \mathrm{mg}, 0.16 \mathrm{mmol}, 79 \%$ yield). (new compound). ${ }^{1} \mathrm{H}$ NMR (400 MHz, $\left.\mathrm{CDCl}_{3}\right): \delta$ 7.86-7.83 (m, 2H), 7.39-7.33 (m, 3H), $5.40(\mathrm{~d}, J=1.2 \mathrm{~Hz}, 1 \mathrm{H}), 4.87(\mathrm{~d}, J=1.2 \mathrm{~Hz}, 1 \mathrm{H}), 4.19-4.03(\mathrm{~m}, 2 \mathrm{H}), 2.41-2.36(\mathrm{~m}$, $1 \mathrm{H}), 2.20-2.12(\mathrm{~m}, 2 \mathrm{H}), 2.05-1.96(\mathrm{~m}, 2 \mathrm{H}), 1.89-1.81(\mathrm{~m}, 3 \mathrm{H}), 1.74-1.64(\mathrm{~m}, 1 \mathrm{H})$, $1.24(\mathrm{t}, J=7.2 \mathrm{~Hz}, 3 \mathrm{H}), 1.02(\mathrm{~d}, J=6.8 \mathrm{~Hz}, 3 \mathrm{H}), 0.93(\mathrm{~d}, J=6.8 \mathrm{~Hz}, 3 \mathrm{H}) ;{ }^{13} \mathrm{C} \mathrm{NMR}$ $\left(125 \mathrm{MHz}, \mathrm{CDCl}_{3}\right): \delta 174.4,170.6,146.8,140.6,129.8,128.7,127.9,118.5,69.9$, $60.5,51.7,35.0,34.0,25.7,24.7,24.7,23.8,14.1$; HRMS: calcd for $\mathrm{C}_{20} \mathrm{H}_{28} \mathrm{NO}_{2}$ 


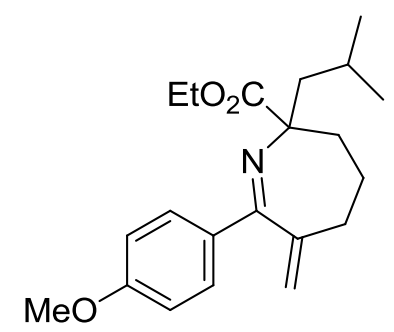

ethyl 2-isobutyl-7-(4-methoxyphenyl)-6-methylene-3,4,5,6-tetrahydro-2 $\mathrm{H}$-azepine-2carboxylate $(\mathbf{3 b})$

Prepared from ethyl 2-isobutyl-2-isocyanohept-6-enoate (47.4 mg, $0.2 \mathrm{mmol}, 1.0$ equiv) and 1-iodo-4-methoxybenzene (70 $\mathrm{mg}, 0.3 \mathrm{mmol}, 1.5$ equiv) according to the general procedure. Column chromatography purification (EtOAc : petroleum ether 15 : 1) furnished the product $\mathbf{3 b}$ as colorless oil (54 mg, $0.16 \mathrm{mmol}, 79 \%$ yield). (new compound). ${ }^{1} \mathrm{H}$ NMR (500 MHz, $\left.\mathrm{CDCl}_{3}\right)$ : $\delta$ 7.82-7.81 (m, 2H), 6.88-6.86 (m, 2H), $5.38(\mathrm{~d}, J=1.0 \mathrm{~Hz}, 1 \mathrm{H}), 4.86(\mathrm{~d}, J=1.0 \mathrm{~Hz}, 1 \mathrm{H}), 4.18-4.02(\mathrm{~m}, 2 \mathrm{H}), 3.82(\mathrm{~d}, J=6.0$ $\mathrm{Hz}, 3 \mathrm{H}), 2.37-2.35(\mathrm{~m}, 1 \mathrm{H}), 2.12-2.11(\mathrm{~m}, 2 \mathrm{H}), 2.03-1.94(\mathrm{~m}, 2 \mathrm{H}), 1.86-1.81(\mathrm{~m}, 3 \mathrm{H})$, 1.71-1.61 (m, 1H), $1.24(\mathrm{t}, J=7.5 \mathrm{~Hz}, 3 \mathrm{H}), 1.01(\mathrm{~d}, J=6.5 \mathrm{~Hz}, 3 \mathrm{H}), 0.92(\mathrm{~d}, J=6.5$ $\mathrm{Hz}, 3 \mathrm{H}) ;{ }^{13} \mathrm{C}$ NMR $\left(125 \mathrm{MHz}, \mathrm{CDCl}_{3}\right): \delta 174.6,170.1,161.2,146.8,133.2,130.2$, 118.2, 113.2, 69.6, 60.4, 55.3, 51.8, 35.1, 34.0, 25.9, 24.7, 24.7, 23.8, 14.1; HRMS: calcd for $\mathrm{C}_{21} \mathrm{H}_{30} \mathrm{NO}_{3}\left(\mathrm{M}^{+}+\mathrm{H}\right)$ 344.2220; found 344.2221.<smiles>C=C1CCCC(COCC)(CC(C)C)N=C1c1ccc(-c2ccccc2)cc1</smiles>

ethyl 7-([1,1'-biphenyl]-4-yl)-2-isobutyl-6-methylene-3,4,5,6-tetrahydro-2H-azepine2-carboxylate $(\mathbf{3 c})$

Prepared from ethyl 2-isobutyl-2-isocyanohept-6-enoate (47.4 mg, $0.2 \mathrm{mmol}, 1.0$ equiv) and 4-iodo-1,1'-biphenyl ( $84 \mathrm{mg}, 0.3 \mathrm{mmol}, 1.5$ equiv) according to the general procedure. Column chromatography purification (EtOAc : petroleum ether $30: 1$ ) furnished the product $3 \mathbf{c}$ as colorless oil $(60 \mathrm{mg}, 0.15 \mathrm{mmol}, 77 \%$ yield). (new compound). ${ }^{1} \mathrm{H}$ NMR (400 MHz, $\left.\mathrm{CDCl}_{3}\right): \delta$ 7.94-7.92 (m, 2H), 7.63-7.58 (m, 4H), 7.47-7.43 (m, 2H), 7.38-7.34 (m, 1H), $5.44(\mathrm{~d}, J=0.8 \mathrm{~Hz}, 1 \mathrm{H}), 4.93(\mathrm{~d}, J=0.8 \mathrm{~Hz}$, 
1H), 4.20-4.05 (m, 2H), 2.43-2.40 (m, 1H), 2.19-2.13 (m, 2H), 2.07-1.98 (m, 2H), 1.90-1.84 (m, 3H), 1.76-1.72 (m, 1H), 1.25 (t, $J=7.2 \mathrm{~Hz}, 3 \mathrm{H}), 1.03(\mathrm{~d}, J=6.4 \mathrm{~Hz}$, $3 \mathrm{H}), 0.95(\mathrm{~d}, J=6.4 \mathrm{~Hz}, 3 \mathrm{H}) ;{ }^{13} \mathrm{C}$ NMR $\left(125 \mathrm{MHz} \mathrm{CDCl}_{3}\right): \delta 174.4,170.2,146.7$, 142.6, 140.8, 139.5, 129.2, 128.8, 127.5, 127.1, 126.7, 118.5, 70.0, 60.5, 51.8 35.1, 34.1, 25.8, 24.7, 24.7, 23.8, 14.1; HRMS: calcd for $\mathrm{C}_{26} \mathrm{H}_{32} \mathrm{NO}_{2}\left(\mathrm{M}^{+}+\mathrm{H}\right)$ 390.2428; found 390.2430 .<smiles>C=C1CCCC(CC(C)C)(OCC)N=C1c1ccc(Cl)cc1</smiles>

ethyl 7-(4-chlorophenyl)-2-isobutyl-6-methylene-3,4,5,6-tetrahydro- $2 \mathrm{H}$-azepine2-carboxylate $(\mathbf{3 d})$

Prepared from ethyl 2-isobutyl-2-isocyanohept-6-enoate $(47.4 \mathrm{mg}, 0.2 \mathrm{mmol}, 1.0$ equiv) and 1-chloro-4-iodobenzene ( $71 \mathrm{mg}, 0.3 \mathrm{mmol}, 1.5$ equiv) according to the general procedure. Column chromatography purification (EtOAc : petroleum ether 30 : 1) furnished the product $\mathbf{3 d}$ as colorless oil (54 mg, $0.16 \mathrm{mmol}, 78 \%$ yield). (new compound). ${ }^{1} \mathrm{H}$ NMR (400 MHz, $\left.\mathrm{CDCl}_{3}\right): \delta 7.78(\mathrm{~d}, J=8.4 \mathrm{~Hz}, 2 \mathrm{H}), 7.31$ (d, $J=8.4$ $\mathrm{Hz}, 2 \mathrm{H}), 5.41(\mathrm{~s}, 1 \mathrm{H}), 4.85(\mathrm{~d}, J=0.8 \mathrm{~Hz}, 1 \mathrm{H}), 4.20-4.02(\mathrm{~m}, 2 \mathrm{H}), 2.40-2.34(\mathrm{~m}, 1 \mathrm{H})$, 2.18-2.10 (m, 2H), 2.03-1.93 (m, 2H), 1.88-1.80 (m, 3H), 1.73-1.67 (m, 1H), 1.23 (t, $J$ $=7.2 \mathrm{~Hz}, 3 \mathrm{H}), 1.00(\mathrm{~d}, J=6.4 \mathrm{~Hz}, 3 \mathrm{H}), 0.92(\mathrm{~d}, J=6.4 \mathrm{~Hz}, 3 \mathrm{H}) ;{ }^{13} \mathrm{C}$ NMR $(125 \mathrm{MHz}$, $\left.\mathrm{CDCl}_{3}\right): \delta 174.1,169.4,146.4,139.0,136.0,130.0,128.1,118.8,70.0,60.5,51.5$, 35.0, 34.0, 25.7, 24.7, 24.7, 23.7, 14.1; HRMS: calcd for $\mathrm{C}_{20} \mathrm{H}_{27} \mathrm{ClNO}_{2}\left(\mathrm{M}^{+}+\mathrm{H}\right)$ 348.1725; found 348.1722.<smiles>C=C1CCCC(CC(C)C)(C(=O)OCC)N=C1c1cccc(Cl)c1</smiles>

ethyl 7-(3-chlorophenyl)-2-isobutyl-6-methylene-3,4,5,6-tetrahydro- $2 \mathrm{H}$-azepine2-carboxylate $(\mathbf{3 e})$

Prepared from ethyl 2-isobutyl-2-isocyanohept-6-enoate (47.4 mg, $0.2 \mathrm{mmol}, 1.0$ 
equiv) and 1-chloro-3-iodobenzene ( $71 \mathrm{mg}, 0.3 \mathrm{mmol}, 1.5$ equiv) according to the general procedure. Column chromatography purification (EtOAc : petroleum ether 30 : 1) furnished the product $3 \mathbf{e}$ as colorless oil (69 $\mathrm{mg}, 0.19 \mathrm{mmol}, 99 \%$ yield). (new compound). ${ }^{1} \mathrm{H}$ NMR (400 MHz, $\left.\mathrm{CDCl}_{3}\right): \delta 7.82$ (t, $\left.J=1.6 \mathrm{~Hz}, 1 \mathrm{H}\right), 7.71$ (d, $J=7.6$ $\mathrm{Hz}, 1 \mathrm{H}), 7.37-7.34(\mathrm{~m}, 1 \mathrm{H}), 7.30-7.26(\mathrm{~m}, 1 \mathrm{H}), 5.42$ (d, $J=0.8 \mathrm{~Hz}, 1 \mathrm{H}), 4.88$ (d, $J=$ $0.8 \mathrm{~Hz}, 1 \mathrm{H}), 4.20-4.04(\mathrm{~m}, 2 \mathrm{H}), 2.39-2.35$ (m, 1H), 2.17-2.10 (m, 2H), 2.02-1.94 (m, 2H), 1.89-1.80 (m, 3H), 1.73-1.69 (m, 1H), $1.24(\mathrm{t}, J=6.8 \mathrm{~Hz}, 3 \mathrm{H}), 1.00(\mathrm{~d}, J=6.4$

$\mathrm{Hz}, 3 \mathrm{H}), 0.92(\mathrm{~d}, J=6.4 \mathrm{~Hz}, 3 \mathrm{H}) ;{ }^{13} \mathrm{C} \mathrm{NMR}\left(125 \mathrm{MHz}, \mathrm{CDCl}_{3}\right): \delta 174.1,169.2,146.2$, 142.4, 134.0, 129.8, 129.1, 128.7, 126.9, 119.0, 70.1, 60.6, 51.5, 35.0, 34.0, 25.6, 24.7, 24.6, 23.7, 14.1; HRMS: calcd for $\mathrm{C}_{20} \mathrm{H}_{27} \mathrm{ClNO}_{2}\left(\mathrm{M}^{+}+\mathrm{H}\right)$ 348.1725; found 348.1723.<smiles>C=C1CCCC(CC(C)C)(OCC)N=C1c1ccc(Br)cc1</smiles>

ethyl 7-(4-bromophenyl)-2-isobutyl-6-methylene-3,4,5,6-tetrahydro- $2 \mathrm{H}$-azepine-2carboxylate $(\mathbf{3 f})$

Prepared from ethyl 2-isobutyl-2-isocyanohept-6-enoate (47.4 mg, $0.2 \mathrm{mmol}, 1.0$ equiv) and 1-bromo-4-iodobenzene ( $84 \mathrm{mg}, 0.3 \mathrm{mmol}, 1.5$ equiv) according to the general procedure. Column chromatography purification (EtOAc : petroleum ether 30 : 1) furnished the product $\mathbf{3 f}$ as colorless oil $(69 \mathrm{mg}, 0.18 \mathrm{mmol}, 90 \%$ yield). (new compound). ${ }^{1} \mathrm{H}$ NMR (400 MHz, $\left.\mathrm{CDCl}_{3}\right): \delta 7.71(\mathrm{~d}, J=8.4 \mathrm{~Hz}, 2 \mathrm{H}), 7.47(\mathrm{~d}, J=8.4$ $\mathrm{Hz}, 2 \mathrm{H}), 5.41(\mathrm{~s}, 1 \mathrm{H}), 4.85(\mathrm{~d}, J=0.8 \mathrm{~Hz}, 1 \mathrm{H}), 4.20-4.02(\mathrm{~m}, 2 \mathrm{H}), 2.40-2.34(\mathrm{~m}, 1 \mathrm{H})$, 2.18-2.10 (m, 2H), 2.03-1.93 (m, 2H), 1.89-1.79 (m, 3H), 1.73-1.67 (m, 1H), 1.23 (t, $J$ $=6.8 \mathrm{~Hz}, 3 \mathrm{H}), 1.00(\mathrm{~d}, J=6.4 \mathrm{~Hz}, 3 \mathrm{H}), 0.92(\mathrm{~d}, J=6.4 \mathrm{~Hz}, 3 \mathrm{H}) ;{ }^{13} \mathrm{C} \mathrm{NMR}(125 \mathrm{MHz}$, $\left.\mathrm{CDCl}_{3}\right): \delta 174.1,169.5,146.3,139.4,131.0,130.3,124.5,118.9,70.0,60.5,51.5$, 35.0, 34.0, 25.7, 24.7, 24.6, 23.7, 14.1; HRMS: calcd for $\mathrm{C}_{20} \mathrm{H}_{27} \mathrm{BrNO}_{2}\left(\mathrm{M}^{+}+\mathrm{H}\right)$ 392.1220; found 392.1222. 
<smiles>C=C1CCCC(COCC)(CC(C)C)N=C1c1ccc(C(C)=O)cc1</smiles>

ethyl 7-(4-bromophenyl)-2-isobutyl-6-methylene-3,4,5,6-tetrahydro-2H-azepine-2carboxylate $(\mathbf{3 g})$

Prepared from ethyl 2-isobutyl-2-isocyanohept-6-enoate (47.4 mg, $0.2 \mathrm{mmol}, 1.0$ equiv) and methyl 4-iodobenzoate (78 $\mathrm{mg}, 0.3 \mathrm{mmol}, 1.5$ equiv) according to the general procedure. Column chromatography purification (EtOAc : petroleum ether 15 : 1) furnished the product $\mathbf{3 g}$ as yellow oil (57 $\mathrm{mg}, 0.15 \mathrm{mmol}, 77 \%$ yield). (new compound). ${ }^{1} \mathrm{H}$ NMR (400 MHz, $\left.\mathrm{CDCl}_{3}\right): \delta 8.01(\mathrm{~d}, J=8.4 \mathrm{~Hz}, 2 \mathrm{H}), 7.88$ (d, $J=8.4$ $\mathrm{Hz}, 2 \mathrm{H}), 5.42$ (s, 1H), 4.85 (s, 1H), 4.19-4.04 (m, 2H), 3.93 (s, 3H), 2.42-2.36 (m, 1H), 2.21-2.11 (m, 2H), 2.03-1.94 (m, 2H), 1.89-1.80 (m, 3H), 1.80-1.72 (m, 1H), 1.23 (t, J $=6.8 \mathrm{~Hz}, 3 \mathrm{H}), 1.00(\mathrm{~d}, J=6.4 \mathrm{~Hz}, 3 \mathrm{H}), 0.92(\mathrm{~d}, J=6.4 \mathrm{~Hz}, 3 \mathrm{H}) ;{ }^{13} \mathrm{C} \mathrm{NMR}(125 \mathrm{MHz}$, $\left.\mathrm{CDCl}_{3}\right): \delta 174.0,169.8,166.9,146.4,144.8,131.1,129.2,128.7,119.1,70.3,60.6$, 52.1, 51.5, 35.0, 34.0, 25.6, 24.7, 24.6, 23.7, 14.1; HRMS: calcd for $\mathrm{C}_{22} \mathrm{H}_{30} \mathrm{NO}_{4}$ $\left(\mathrm{M}^{+}+\mathrm{H}\right)$ 372.2169; found 372.2171.

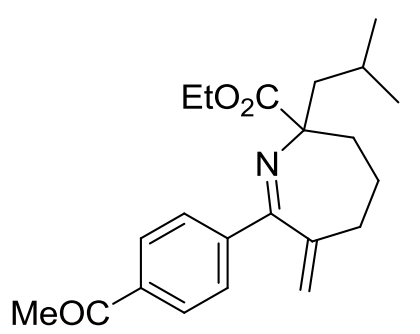

ethyl 7-(4-acetylphenyl)-2-isobutyl-6-methylene-3,4,5,6-tetrahydro-2H-azepine-2carboxylate $(\mathbf{3 h})$

Prepared from ethyl 2-isobutyl-2-isocyanohept-6-enoate (47.4 mg, $0.2 \mathrm{mmol}, 1.0$ equiv) and 1-(4-iodophenyl)ethanone (74 mg, $0.3 \mathrm{mmol}, 1.5$ equiv) according to the general procedure. Column chromatography purification (EtOAc : petroleum ether 15 : 1) furnished the product $3 \mathbf{h}$ as yellow oil $(70 \mathrm{mg}, 0.19 \mathrm{mmol}, 99 \%$ yield). (new compound). ${ }^{1} \mathrm{H}$ NMR (400 MHz, $\left.\mathrm{CDCl}_{3}\right): \delta 7.94-7.89(\mathrm{~m}, 4 \mathrm{H}), 5.43(\mathrm{~d}, J=1.2 \mathrm{~Hz}$, $1 \mathrm{H}), 4.85(\mathrm{~d}, J=1.2 \mathrm{~Hz}, 1 \mathrm{H}), 4.19-4.04(\mathrm{~m}, 2 \mathrm{H}), 2.60$ (s, 3H), 2.41-2.36 (m, 1H), 2.19-2.10 (m, 2H), 2.03-1.94 (m, 2H), 1.90-1.81 (m, 3H), 1.74-1.70 (m, 1H), 1.23 (t, J $=7.2 \mathrm{~Hz}, 3 \mathrm{H}), 1.00(\mathrm{~d}, J=6.4 \mathrm{~Hz}, 3 \mathrm{H}), 0.92(\mathrm{~d}, J=6.4 \mathrm{~Hz}, 3 \mathrm{H}) ;{ }^{13} \mathrm{C} \mathrm{NMR}(125 \mathrm{MHz}$, 
$\left.\mathrm{CDCl}_{3}\right): \delta 197.8,174.0,169.7,146.4,144.8,137.8,128.9,127.9,119.0,70.3,60.6$, 51.5, 35.0, 34.0, 26.7, 25.6, 24.7, 24.6, 23.7, 14.1; HRMS: calcd for $\mathrm{C}_{22} \mathrm{H}_{30} \mathrm{NO}_{3}$ $\left(\mathrm{M}^{+}+\mathrm{H}\right)$ 356.2220; found 356.2220.<smiles>C=C1CCCC(COCC)(CC(C)C)N=C1c1ccc(C#N)cc1</smiles>

ethyl 7-(4-cyanophenyl)-2-isobutyl-6-methylene-3,4,5,6-tetrahydro- $2 \mathrm{H}$-azepine-2carboxylate $(\mathbf{3 i})$

Prepared from ethyl 2-isobutyl-2-isocyanohept-6-enoate (47.4 mg, $0.2 \mathrm{mmol}, 1.0$ equiv) and 4-iodobenzonitrile ( $69 \mathrm{mg}, 0.3 \mathrm{mmol}, 1.5$ equiv) according to the general procedure. Column chromatography purification (EtOAc : petroleum ether $15: 1$ ) furnished the product $3 \mathbf{i}$ as yellow oil (67 $\mathrm{mg}, 0.19 \mathrm{mmol}, 99 \%$ yield). (new compound). ${ }^{1} \mathrm{H}$ NMR (400 MHz, $\mathrm{CDCl}_{3}$ ): $\delta 7.92$ (d, $\left.J=8.4 \mathrm{~Hz}, 2 \mathrm{H}\right), 6.30$ (d, $J=8.4$ $\mathrm{Hz}, 2 \mathrm{H}), 5.45(\mathrm{~d}, J=0.8 \mathrm{~Hz}, 1 \mathrm{H}), 4.85(\mathrm{~d}, J=0.8 \mathrm{~Hz}, 1 \mathrm{H}), 4.19-4.05(\mathrm{~m}, 2 \mathrm{H})$, 2.40-2.36 (m, 1H), 2.18-2.10 (m, 2H), 2.00-1.78 (m, 5H), 1.74-1.67 (m, 1H), $1.23(\mathrm{t}, J$ $=7.2 \mathrm{~Hz}, 3 \mathrm{H}), 0.99(\mathrm{~d}, J=6.4 \mathrm{~Hz}, 3 \mathrm{H}), 0.91(\mathrm{~d}, J=6.4 \mathrm{~Hz}, 3 \mathrm{H}) ;{ }^{13} \mathrm{C} \mathrm{NMR}(125 \mathrm{MHz}$, $\left.\mathrm{CDCl}_{3}\right): \delta 173.7,168.9,145.9,144.6,131.7,129.2,119.4,118.7,113.2,70.5,60.7$, 51.3, 35.0, 34.0, 25.5, 24.7, 24.6, 23.6, 14.1; HRMS: calcd for $\mathrm{C}_{21} \mathrm{H}_{27} \mathrm{~N}_{2} \mathrm{O}_{2}\left(\mathrm{M}^{+}+\mathrm{H}\right)$ 339.2067; found 339.2071.<smiles>C=C1CCCC(COCC)(CC(C)C)N=C1c1ccc([N+](=O)[O-])cc1</smiles>

ethyl 2-isobutyl-6-methylene-7-(4-nitrophenyl)-3,4,5,6-tetrahydro-2 $H$-azepine-2carboxylate $(\mathbf{3 j})$

Prepared from ethyl 2-isobutyl-2-isocyanohept-6-enoate (47.4 mg, $0.2 \mathrm{mmol}, 1.0$ equiv) and 1-iodo-4-nitrobenzene (74 mg, $0.3 \mathrm{mmol}, 1.5$ equiv) according to the general procedure. Column chromatography purification (EtOAc : petroleum ether 15 : 1) furnished the product $\mathbf{3 j}$ as yellow oil (69 $\mathrm{mg}, 0.19 \mathrm{mmol}, 97 \%$ yield). (new 
compound). ${ }^{1} \mathrm{H}$ NMR (400 MHz, $\left.\mathrm{CDCl}_{3}\right): \delta 8.21-8.17(\mathrm{~m}, 2 \mathrm{H}), 8.00-7.96(\mathrm{~m}, 2 \mathrm{H})$, $5.47(\mathrm{~d}, J=0.8 \mathrm{~Hz}, 1 \mathrm{H}), 4.87(\mathrm{~d}, J=0.8 \mathrm{~Hz}, 2 \mathrm{H}), 4.20-4.06(\mathrm{~m}, 2 \mathrm{H}), 2.41-2.38(\mathrm{~m}$, $1 \mathrm{H}), 2.18-2.11(\mathrm{~m}, 2 \mathrm{H}), 2.02-1.82(\mathrm{~m}, 5 \mathrm{H}), 1.76-1.71(\mathrm{~m}, 1 \mathrm{H}), 1.24(\mathrm{t}, J=7.2 \mathrm{~Hz}$, $3 \mathrm{H}), 1.00(\mathrm{~d}, J=6.4 \mathrm{~Hz}, 3 \mathrm{H}), 0.92(\mathrm{~d}, J=6.4 \mathrm{~Hz}, 3 \mathrm{H}) ;{ }^{13} \mathrm{C} \mathrm{NMR}\left(125 \mathrm{MHz}, \mathrm{CDCl}_{3}\right)$ : $\delta 173.6,168.6,148.6,146.3,146.0,129.5,123.1,119.5,70.6,60.7,51.2,35.0,34.0$, 25.5, 24.7, 24.6, 23.6, 14.1; HRMS: calcd for $\mathrm{C}_{20} \mathrm{H}_{27} \mathrm{~N}_{2} \mathrm{O}_{4}\left(\mathrm{M}^{+}+\mathrm{H}\right)$ 359.1965; found 359.1963.<smiles>C=C1CCCC(CC(C)C)(C(=O)OCC)N=C1c1cccc(C=O)c1</smiles>

ethyl 7-(3-formylphenyl)-2-isobutyl-6-methylene-3,4,5,6-tetrahydro-2 $\mathrm{H}$-azepine-2carboxylate $(\mathbf{3 k})$

Prepared from ethyl 2-isobutyl-2-isocyanohept-6-enoate (47.4 mg, $0.2 \mathrm{mmol}, 1.0$ equiv) and 3-iodobenzaldehyde (70 $\mathrm{mg}, 0.3 \mathrm{mmol}, 1.5$ equiv) according to the general procedure. Column chromatography purification (EtOAc : petroleum ether $15: 1$ ) furnished the product $\mathbf{3 k}$ as colorless oil $(68 \mathrm{mg}, 0.19 \mathrm{mmol}, 99 \%$ yield). (new compound). ${ }^{1} \mathrm{H}$ NMR (400 MHz, $\left.\mathrm{CDCl}_{3}\right): \delta 10.0(\mathrm{~s}, 1 \mathrm{H}), 8.29(\mathrm{t}, J=1.2 \mathrm{~Hz}, 1 \mathrm{H})$, $8.16(\mathrm{ddd}, J=8.0,1.2,1.2 \mathrm{~Hz}, 1 \mathrm{H}), 7.91(\mathrm{ddd}, J=8.0,1.2,1.2 \mathrm{~Hz}, 1 \mathrm{H}), 7.51(\mathrm{t}, J=$ $7.6 \mathrm{~Hz}, 1 \mathrm{H}), 5.46(\mathrm{~s}, 1 \mathrm{H}), 4.89$ (d, $J=0.8 \mathrm{~Hz}, 1 \mathrm{H}), 4.20-4.05(\mathrm{~m}, 2 \mathrm{H}), 2.43-2.38(\mathrm{~m}$, $1 \mathrm{H}), 2.21-2.11(\mathrm{~m}, 2 \mathrm{H}), 2.03-1.95(\mathrm{~m}, 2 \mathrm{H}), 1.90-1.81(\mathrm{~m}, 3 \mathrm{H}), 1.75-1.71(\mathrm{~m}, 1 \mathrm{H})$, $1.24(\mathrm{t}, J=7.2 \mathrm{~Hz}, 3 \mathrm{H}), 1.00(\mathrm{~d}, J=6.4 \mathrm{~Hz}, 3 \mathrm{H}), 0.93(\mathrm{~d}, J=6.4 \mathrm{~Hz}, 3 \mathrm{H}) ;{ }^{13} \mathrm{C} \mathrm{NMR}$ $\left(125 \mathrm{MHz}, \mathrm{CDCl}_{3}\right): \delta$ 192.2, 174.0, 169.3, 146.1, 141.6, 136.2, 134.5, 130.5, 130.5, 128.7, 119.3, 70.2, 60.6, 51.4, 35.0, 34.0, 25.6, 24.7, 24.6, 23.7, 14.1; HRMS: calcd for $\mathrm{C}_{21} \mathrm{H}_{28} \mathrm{NO}_{3}\left(\mathrm{M}^{+}+\mathrm{H}\right)$ 342.2064; found 342.2065.<smiles>C=C1CCCC(CC(C)C)(C(=O)OCC)N=C1c1cc(Br)cc(Br)c1</smiles> 
ethyl 7-(3,5-dibromophenyl)-2-isobutyl-6-methylene-3,4,5,6-tetrahydro-2H-azepine2-carboxylate (31)

Prepared from ethyl 2-isobutyl-2-isocyanohept-6-enoate (47.4 mg, $0.2 \mathrm{mmol}, 1.0$ equiv) and 1,3-dibromo-5-iodobenzene (108 $\mathrm{mg}, 0.3 \mathrm{mmol}, 1.5$ equiv) according to the general procedure. Column chromatography purification (EtOAc : petroleum ether $30: 1$ ) furnished the product $\mathbf{3 l}$ as yellow oil (79 $\mathrm{mg}, 0.17 \mathrm{mmol}, 84 \%$ yield). (new compound). ${ }^{1} \mathrm{H}$ NMR (400 MHz, $\left.\mathrm{CDCl}_{3}\right): \delta 7.89$ (d, $\left.J=2.0 \mathrm{~Hz}, 2 \mathrm{H}\right), 7.67$ (t, $J=1.6$ $\mathrm{Hz}, 1 \mathrm{H}), 5.45$ (s, 1H), 4.89 (s, 1H), 4.19-4.05 (m, 2H), 2.39-2.33 (m, 1H), 2.16-2.10 (m, 2H), 1.98-1.78 (m, 5H), 1.71-1.65 (m, 1H), 1.24 (t, $J=7.2 \mathrm{~Hz}, 3 \mathrm{H}), 0.99$ (d, $J=$ $6.4 \mathrm{~Hz}, 3 \mathrm{H}), 0.92(\mathrm{~d}, J=6.4 \mathrm{~Hz}, 3 \mathrm{H}) ;{ }^{13} \mathrm{C} \mathrm{NMR}\left(125 \mathrm{MHz}, \mathrm{CDCl}_{3}\right): \delta 173.8,167.8$, 145.6, 143.9, 135.1, 130.5, 122.6, 119.6, 70.3, 60.8, 51.3, 34.9, 33.9, 25.6, 24.7, 24.7, 23.8, 14.2; HRMS: calcd for $\mathrm{C}_{20} \mathrm{H}_{26} \mathrm{Br}_{2} \mathrm{NO}_{2}\left(\mathrm{M}^{+}+\mathrm{H}\right)$ 470.0325; found 470.0323 .

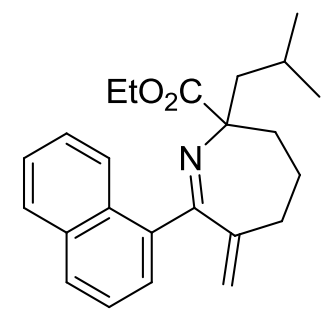

ethyl 2-isobutyl-6-methylene-7-(naphthalen-1-yl)-3,4,5,6-tetrahydro-2H-azepine-2carboxylate $(\mathbf{3 m})$

Prepared from ethyl 2-isobutyl-2-isocyanohept-6-enoate $(47.4 \mathrm{mg}, 0.2 \mathrm{mmol}, 1.0$ equiv) and 1-iodonaphthalene ( $76 \mathrm{mg}, 0.3 \mathrm{mmol}, 1.5$ equiv) according to the general procedure. Column chromatography purification (EtOAc : petroleum ether $30: 1$ ) furnished the product $\mathbf{3 m}$ as colorless oil $(43 \mathrm{mg}, 0.17 \mathrm{mmol}, 59 \%$ yield). (new compound). ${ }^{1} \mathrm{H}$ NMR (400 MHz, $\left.\mathrm{CDCl}_{3}\right): \delta 8.33-8.31(\mathrm{~m}, 1 \mathrm{H}), 7.84-7.82(\mathrm{~m}, 2 \mathrm{H})$, 7.51-7.42 (m, 4H), $5.27(\mathrm{~s}, 1 \mathrm{H}), 4.83(\mathrm{~s}, 1 \mathrm{H}), 4.26-4.15(\mathrm{~m}, 2 \mathrm{H}), 2.65-2.59(\mathrm{~m}, 1 \mathrm{H})$, 2.49-2.42 (m, 1H), 2.34-2.30 (m, 1H), 2.10-2.04 (m, 1H), 2.03-2.00 (m, 2H), 1.97-1.84 (m, 3H), $1.31(\mathrm{t}, J=7.6 \mathrm{~Hz}, 3 \mathrm{H}), 0.95-0.92(\mathrm{~m}, 6 \mathrm{H}) ;{ }^{13} \mathrm{C} \mathrm{NMR}(125 \mathrm{MHz}$, $\left.\mathrm{CDCl}_{3}\right): \delta 174.2,171.7,148.7,141.4,133.9,131.5,128.8,128.1,127.0,126.6,125.9$, 125.6, 124.8, 120.8, 70.8, 60.8, 52.7, 35.3, 34.2, 24.9, 24.7, 24.4, 23.6, 14.1; HRMS: calcd for $\mathrm{C}_{24} \mathrm{H}_{30} \mathrm{NO}_{2}\left(\mathrm{M}^{+}+\mathrm{H}\right)$ 364.2271; found 364.2273. 
<smiles>C=C1CCCC(CC(=O)OCC)(CC(C)C)N=C1c1ccc2c(c1)OCO2</smiles>

ethyl 7-(benzo[d][1,3]dioxol-5-yl)-2-isobutyl-6-methylene-3,4,5,6-tetrahydro-2 $\mathrm{H}$ azepine-2-carboxylate (3n)

Prepared from ethyl 2-isobutyl-2-isocyanohept-6-enoate $(47.4 \mathrm{mg}, 0.2 \mathrm{mmol}, 1.0$ equiv) and 5-bromobenzo[d][1,3]dioxole (49 $\mathrm{mg}, 0.3 \mathrm{mmol}, 1.5$ equiv) according to the procedure A. Column chromatography purification (EtOAc : petroleum ether 15 : 1) furnished the product $3 \mathrm{n}$ as colorless oil $(79 \mathrm{mg}, 0.13 \mathrm{mmol}, 66 \%$ yield). (new compound). ${ }^{1} \mathrm{H}$ NMR (500 MHz, $\left.\mathrm{CDCl}_{3}\right): \delta 7.52$ (d, $\left.J=1.0 \mathrm{~Hz}, 1 \mathrm{H}\right), 7.35(\mathrm{dd}, J=6.4$, $1.5 \mathrm{~Hz}, 1 \mathrm{H}), 6.81(\mathrm{~d}, J=6.4 \mathrm{~Hz}, 1 \mathrm{H}), 6.02(\mathrm{~d}, J=1.0 \mathrm{~Hz}, 2 \mathrm{H}), 5.42(\mathrm{~s}, 1 \mathrm{H}), 4.91(\mathrm{~s}$, $1 \mathrm{H}), 4.23-4.05(\mathrm{~m}, 2 \mathrm{H}), 2.41-2.36(\mathrm{~m}, 1 \mathrm{H}), 2.18-2.15(\mathrm{~m}, 2 \mathrm{H}), 2.06-1.98(\mathrm{~m}, 3 \mathrm{H})$, 1.89-1.85 (m, 2H), 1.74-1.70 (m, 1H), 1.27 (t, $J=7.0 \mathrm{~Hz}, 3 \mathrm{H}), 1.04$ (d, $J=7.0 \mathrm{~Hz}$, $3 \mathrm{H}), 0.96(\mathrm{~d}, J=7.0 \mathrm{~Hz}, 3 \mathrm{H}) ;{ }^{13} \mathrm{C} \mathrm{NMR}\left(125 \mathrm{MHz}, \mathrm{CDCl}_{3}\right): \delta 174.5,169.8,149.3$, $147.6,146.7,135.1,123.9,118.4,108.6,107.3,101.3,70.0,60.5,51.8,35.1,34.0$, 25.9, 24.7, 24.7, 23.8, 14.1; HRMS: calcd for $\mathrm{C}_{21} \mathrm{H}_{28} \mathrm{NO}_{4}\left(\mathrm{M}^{+}+\mathrm{H}\right)$ 358.2013; found 358.2011<smiles>C=C1CCCC(CC(C)C)(C(=O)OCC)N=C1c1ccc2ncccc2c1</smiles>

ethyl 2-isobutyl-6-methylene-7-(quinolin-6-yl)-3,4,5,6-tetrahydro-2 $H$-azepine-2carboxylate (3o)

Prepared from ethyl 2-isobutyl-2-isocyanohept-6-enoate (47.4 mg, $0.2 \mathrm{mmol}, 1.0$ equiv) and 6-iodoquinoline ( $77 \mathrm{mg}, 0.3 \mathrm{mmol}, 1.5$ equiv) according to the general procedure. Column chromatography purification (EtOAc : petroleum ether $15: 1$ ) furnished the product 30 as yellow oil (72 $\mathrm{mg}, 0.19 \mathrm{mmol}, 99 \%$ yield). (new compound). ${ }^{1} \mathrm{H} \mathrm{NMR}\left(400 \mathrm{MHz}, \mathrm{CDCl}_{3}\right): \delta 8.92(\mathrm{dd}, J=4.0,1.6 \mathrm{~Hz}, 1 \mathrm{H}), 8.37$ (dd, $J$ $=8.4,1.2 \mathrm{~Hz}, 1 \mathrm{H}), 8.20-8.18(\mathrm{~m}, 2 \mathrm{H}), 8.09(\mathrm{~d}, J=8.8 \mathrm{~Hz}, 1 \mathrm{H}), 7.41(\mathrm{dd}, J=4.4,4.0$ 
$\mathrm{Hz}, 1 \mathrm{H}), 5.50(\mathrm{~s}, 1 \mathrm{H}), 4.94(\mathrm{~d}, J=1.2 \mathrm{~Hz}, 1 \mathrm{H}), 4.22-4.06(\mathrm{~m}, 2 \mathrm{H}), 2.47-2.42(\mathrm{~m}, 1 \mathrm{H})$, 2.25-2.15 (m, 2H), 2.09-2.00 (m, 2H), 1.93-1.86 (m, 3H), 1.78-1.73 (m, 1H), $1.25(\mathrm{t}, J$ $=7.2 \mathrm{~Hz}, 3 \mathrm{H}), 1.05(\mathrm{~d}, J=6.4 \mathrm{~Hz}, 3 \mathrm{H}), 0.96(\mathrm{~d}, J=6.4 \mathrm{~Hz}, 3 \mathrm{H}) ;{ }^{13} \mathrm{C} \mathrm{NMR}(125 \mathrm{MHz}$, $\left.\mathrm{CDCl}_{3}\right): \delta 174.2,169.8,151.0,149.2,146.4,138.4,136.9,129.4,129.1,128.8,127.6$, 121.2, 118.9, 70.2, 60.6, 51.6, 35.0, 34.0, 25.8, 24.7, 24.7, 23.8, 14.1; HRMS: calcd for $\mathrm{C}_{23} \mathrm{H}_{29} \mathrm{~N}_{2} \mathrm{O}_{2}\left(\mathrm{M}^{+}+\mathrm{H}\right)$ 365.2224; found 365.2223.<smiles>C=C1CCCC(COCC)(CC(C)C)N=C1c1cccnc1</smiles>

ethyl 2-isobutyl-6-methylene-7-(pyridin-3-yl)-3,4,5,6-tetrahydro-2H-azepine-2carboxylate $(\mathbf{3 p})$

Prepared from ethyl 2-isobutyl-2-isocyanohept-6-enoate (47.4 mg, $0.2 \mathrm{mmol}, 1.0$ equiv) and 3-iodopyridine (62 $\mathrm{mg}, 0.3 \mathrm{mmol}, 1.5$ equiv) according to the general procedure. Column chromatography purification (EtOAc : petroleum ether $15: 1$ ) furnished the product $3 \mathbf{p}$ as yellow oil $(55 \mathrm{mg}, 0.17 \mathrm{mmol}, 87 \%$ yield). (new compound). ${ }^{1} \mathrm{H} \mathrm{NMR}\left(400 \mathrm{MHz}, \mathrm{CDCl}_{3}\right): \delta 9.00$ (d, $\left.J=1.6 \mathrm{~Hz}, 1 \mathrm{H}\right), 8.62$ (dd, $J=4.8$, $1.6 \mathrm{~Hz}, 1 \mathrm{H}), 8.15(\mathrm{ddd}, J=8.0,2.0,2.0 \mathrm{~Hz}, 1 \mathrm{H}), 7.32-7.28(\mathrm{~m}, 1 \mathrm{H}), 5.48(\mathrm{~d}, J=0.8$ $\mathrm{Hz}, 1 \mathrm{H}), 4.92$ (d, $J=0.8 \mathrm{~Hz}, 1 \mathrm{H}), 4.21-4.07$ (m, 2H), 2.44-2.39 (m, 1H), 2.22-2.13 (m, $2 \mathrm{H}), 2.05-1.72(\mathrm{~m}, 6 \mathrm{H}), 1.26(\mathrm{t}, J=7.2 \mathrm{~Hz}, 3 \mathrm{H}), 1.01(\mathrm{~d}, J=6.4 \mathrm{~Hz}, 3 \mathrm{H}), 0.93(\mathrm{~d}, J=$ $6.4 \mathrm{~Hz}, 3 \mathrm{H}) ;{ }^{13} \mathrm{C} \mathrm{NMR}\left(125 \mathrm{MHz}, \mathrm{CDCl}_{3}\right): \delta 173.9,168.5,150.6,150.3,146.1,136.2$, 135.9, 122.9, 119.5, 70.4, 60.7, 51.5, 35.2, 34.1, 25.6, 24.7, 24.7, 23.6, 14.1; HRMS: calcd for $\mathrm{C}_{19} \mathrm{H}_{27} \mathrm{~N}_{2} \mathrm{O}_{2}\left(\mathrm{M}^{+}+\mathrm{H}\right) 315.2067$; found 315.2067.

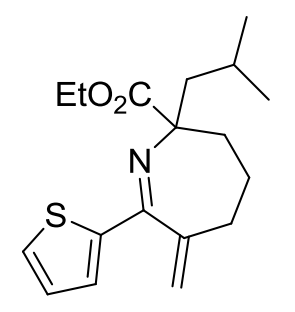

ethyl

2-isobutyl-6-methylene-7-(thiophen-2-yl)-3,4,5,6-tetrahydro-2H-azepine-2-carboxylat e (3q) 
Prepared from ethyl 2-isobutyl-2-isocyanohept-6-enoate (47.4 mg, $0.2 \mathrm{mmol}, 1.0$ equiv) and 2-iodothiophene ( $63 \mathrm{mg}, 0.3 \mathrm{mmol}, 1.5$ equiv) according to the general procedure. Column chromatography purification (EtOAc : petroleum ether $15: 1$ ) furnished the product $\mathbf{3 q}$ as colorless oil $(35 \mathrm{mg}, 0.11 \mathrm{mmol}, 55 \%$ yield). (new compound). ${ }^{1} \mathrm{H}$ NMR (400 MHz, $\left.\mathrm{CDCl}_{3}\right)$ : $\delta$ 7.37-7.36 (m, $\left.1 \mathrm{H}\right)$, 7.28-7.26 (m, $\left.1 \mathrm{H}\right)$, 7.00-6.98 (m, 1H), $5.40(\mathrm{~s}, 1 \mathrm{H}), 5.11(\mathrm{~d}, J=1.2 \mathrm{~Hz}, 1 \mathrm{H}), 4.18-4.05(\mathrm{~m}, 2 \mathrm{H})$, 2.35-2.30 (m, 1H), 2.13-2.07 (m, 2H), 2.00-1.91 (m, 1H), 1.90-1.80 (m, 3H), 1.77-1.64 (m, 1H), $1.23(\mathrm{t}, J=7.2 \mathrm{~Hz}, 3 \mathrm{H}), 1.01(\mathrm{~d}, J=6.4 \mathrm{~Hz}, 3 \mathrm{H}), 0.91(\mathrm{~d}, J=6.4$ $\mathrm{Hz}, 3 \mathrm{H}) ;{ }^{13} \mathrm{C} \mathrm{NMR}\left(125 \mathrm{MHz}, \mathrm{CDCl}_{3}\right): \delta 174.2,165.2,148.1,145.9,123.0,129.5$, 127.0, 118.3, 70.1, 60.6, 51.6, 35.6, 33.9, 26.0, 24.8, 24.7, 23.8, 14.2; HRMS: calcd for $\mathrm{C}_{18} \mathrm{H}_{26} \mathrm{NO}_{2} \mathrm{~S}\left(\mathrm{M}^{+}+\mathrm{H}\right) 320.1679$; found 320.1680 .

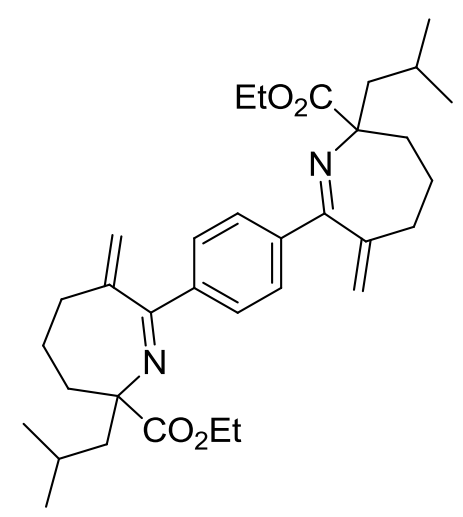

diethyl 7,7'-(1,4-phenylene)bis(2-isobutyl-6-methylene-3,4,5,6-tetrahydro-2 $\mathrm{H}$ azepine-2-carboxylate) (3r)

Procedure: An oven-dried $25 \mathrm{~mL}$ Schlenk tube charged with $\mathrm{Pd}(\mathrm{OAc})_{2}(0.02$ mmol, $4.5 \mathrm{mg}), \mathrm{PPh}_{3}(0.04 \mathrm{mmol}, 10.5 \mathrm{mg}), 1,4$-diiodobenzene $(0.1 \mathrm{mmol}, 33 \mathrm{mg})$ and CsOPiv (0.24 mmol, $56.0 \mathrm{mg}$ ) was vacuumed and refilled with Ar for 3 times. Then the tube was placed in an oil-bath at $80{ }^{\circ} \mathrm{C}$. A solution of ethyl 2-isobutyl-2-isocyanohept-6-enoate $(47.4 \mathrm{mg}, 0.2 \mathrm{mmol})$ in $1.0 \mathrm{~mL}$ of toluene was added dropwise with a syringe pump within $1 \mathrm{~h}$. The crude reaction mixture was extracted with DCM $(20 \mathrm{~mL} \times 3)$ and washed with brine $(20 \mathrm{~mL})$. The organic phase was concentrated in vacuo and the residue was purified by silica gel flash column chromatography to afford $\mathbf{3 r}$ as colorless oil (42 $\mathrm{mg}, 0.15 \mathrm{mmol}, 76 \%$ yield). (new compound). ${ }^{1} \mathrm{H}$ NMR (400 MHz, $\left.\mathrm{CDCl}_{3}\right): \delta 7.81$ (s, 4H), 5.39 (s, 2H), 4.85 (s, 2H), 4.19-4.00 (m, 4H), 2.41-2.35 (m, 2H), 2.19-2.12 (m, 4H), 2.06-1.94 (m, 4H), 1.88-1.79 (m, 6H), 1.74-1.68 (m, 2H), $1.22(\mathrm{t}, J=7.2 \mathrm{~Hz}, 6 \mathrm{H}), 1.00(\mathrm{~d}, J=6.4 \mathrm{~Hz}$, 
6H), $0.92(\mathrm{~d}, J=6.4 \mathrm{~Hz}, 6 \mathrm{H}) ;{ }^{13} \mathrm{C} \mathrm{NMR}\left(125 \mathrm{MHz}, \mathrm{CDCl}_{3}\right): \delta 174.4,170.3,146.7$, 141.9, 128.3, 118.7, 70.1, 60.5, 51.8, 35.1, 34.0, 25.7, 24.7, 24.7, 23.8, 14.1; HRMS: calcd for $\mathrm{C}_{34} \mathrm{H}_{49} \mathrm{~N}_{2} \mathrm{O}_{4}\left(\mathrm{M}^{+}+\mathrm{H}\right)$ 549.3687; found 549.3685.

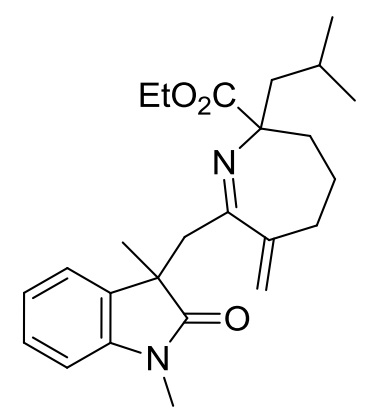

ethyl 7-((1,3-dimethyl-2-oxoindolin-3-yl)methyl)-2-isobutyl-6-methylene-3,4,5,6tetrahydro- $2 H$-azepine-2-carboxylate $(3 \mathbf{s})$

Prepared from ethyl 2-isobutyl-2-isocyanohept-6-enoate (47.4 mg, $0.2 \mathrm{mmol}, 1.0$ equiv) and $N$-(2-iodophenyl)- $N$-methylmethacrylamide (91 $\mathrm{mg}, 0.3 \mathrm{mmol}, 1.5$ equiv) according to the general procedure. Column chromatography purification (EtOAc : petroleum ether $10: 1)$ furnished the product $3 \mathrm{~s}$ as yellow solid $(50 \mathrm{mg}, 0.12 \mathrm{mmol}$, $61 \%$ yield). (new compound). ${ }^{1} \mathrm{H}$ NMR (500 $\left.\mathrm{MHz} \mathrm{CDCl}_{3}\right): \delta$ 7.22-7.17 (m, 2H), 7.00-6.98 (m, 1H), 6.79-6.76 (m, 1H), 5.03-5.01 (m, 1H), 4.92-4.91 (m, 1H), 4.06-3.90 (m, 2H), 3.32-3.28 (m, 1H), 3.19-3.17 (m, 2H), 3.03-2.98 (m, 1H), $1.84-1.82(\mathrm{~m}, 1 \mathrm{H}), 1.69-1.54(\mathrm{~m}, 4 \mathrm{H}), 1.52-1.32(\mathrm{~m}, 6 \mathrm{H}), 1.26-1.10(\mathrm{~m}, 5 \mathrm{H})$, 0.80-0.68 (m, 6H); ${ }^{13} \mathrm{C}$ NMR (125 MHz, $\left.\mathrm{CDCl}_{3}\right): \delta 180.4,174.4,168.5,148.4,143.8$, 134.0, 127.3, 122.8, 121.8, 115.7, 107.6, 69.4, 60.4, 49.0, 48.6, 46.9, 34.4, 32.9, 26.2, 25.7, 24.3, 24.3, 24.0, 23.5, 14.1; HRMS: calcd for $\mathrm{C}_{25} \mathrm{H}_{35} \mathrm{~N}_{2} \mathrm{O}_{3}\left(\mathrm{M}^{+}+\mathrm{H}\right)$ 411.2642; found 411.2645 .<smiles>C=C1CCCC(C)(C(C)C)N=C1c1ccccc1</smiles>

methyl 2-isopropyl-6-methylene-7-phenyl-3,4,5,6-tetrahydro-2 $H$-azepine-2carboxylate (4a)

Prepared from methyl 2-isocyano-2-isopropylhept-6-enoate (42 mg, $0.2 \mathrm{mmol}$, 1.0 equiv) and iodobenzene ( $61 \mathrm{mg}, 0.3 \mathrm{mmol}, 1.5$ equiv) according to the general 
procedure. Column chromatography purification (EtOAc : petroleum ether $30: 1$ ) furnished the product $\mathbf{4 a}$ as colorless oil (56 $\mathrm{mg}, 0.19 \mathrm{mmol}, 99 \%$ yield). (new compound). ${ }^{1} \mathrm{H} \mathrm{NMR}\left(400 \mathrm{MHz}, \mathrm{CDCl}_{3}\right.$ ): $\delta 7.90$ (dd, $\left.J=8.0,1.6 \mathrm{~Hz}, 2 \mathrm{H}\right), 7.42-7.26$ (m, 3H), 5.39 (s, 1H), 4.87 (d, $J=1.2 \mathrm{~Hz}, 1 \mathrm{H}), 3.60$ (s, 3H), 2.45-2.39 (m, 1H), 2.22-2.04 (m, 3H), 1.86-1.79 (m, 2H), 1.71-1.64 (m, 1H), 1.07 (d, $J=6.4 \mathrm{~Hz}, 3 \mathrm{H})$, $1.04(\mathrm{~d}, J=6.4 \mathrm{~Hz}, 3 \mathrm{H}) ;{ }^{13} \mathrm{C} \mathrm{NMR}\left(125 \mathrm{MHz} \mathrm{CDCl}_{3}\right): \delta 174.2,170.9,146.2,140.2$, 129.8, 128.6, 127.8, 118.0, 72.6, 51.1, 40.2, 33.6, 31.8, 26.2, 18.4, 16.9; HRMS: calcd for $\mathrm{C}_{18} \mathrm{H}_{24} \mathrm{NO}_{2}\left(\mathrm{M}^{+}+\mathrm{H}\right) 286.1802$; found 286.1800.

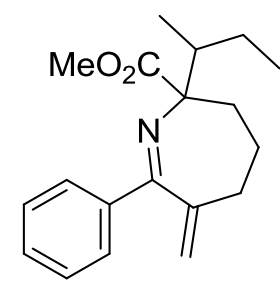

methyl 2-(sec-butyl)-6-methylene-7-phenyl-3,4,5,6-tetrahydro-2H-azepine-2carboxylate $\mathbf{( 4 b )}$

Prepared from methyl 2-(sec-butyl)-2-isocyanohept-6-enoate (45 mg, $0.2 \mathrm{mmol}$, 1.0 equiv) and iodobenzene ( $61 \mathrm{mg}, 0.3 \mathrm{mmol}, 1.5$ equiv) according to the general procedure. Column chromatography purification (EtOAc : petroleum ether $30: 1$ ) furnished the product $\mathbf{4 b}$ as colorless oil $(55 \mathrm{mg}, 0.18 \mathrm{mmol}, 92 \%$ yield). (new compound). ${ }^{1} \mathrm{H}$ NMR (400 MHz, $\left.\mathrm{CDCl}_{3}\right)$ : $\delta$ 7.90-7.88 (m, 2H), 7.40-7.33 (m, 3H), $5.38(\mathrm{~s}, 1 \mathrm{H}), 4.86(\mathrm{~d}, J=1.2 \mathrm{~Hz}, 1 \mathrm{H}), 3.60(\mathrm{~s}, 3 \mathrm{H}), 2.44-2.38(\mathrm{~m}, 1 \mathrm{H}), 2.15-2.07(\mathrm{~m}$, 2H), 1.94-1.64 (m, 5H), 1.40-1.21 (m, 1H), 1.07-0.94 (m, 6H); ${ }^{13} \mathrm{C}$ NMR (125 MHz, $\left.\mathrm{CDCl}_{3}\right): \delta 174.3,170.7,146.2,140.1,129.8,128.6,127.8,117.9,73.2,51.1,47.3$, 33.4, 31.4, 25.8, 24.8, 13.0, 12.7; HRMS: calcd for $\mathrm{C}_{19} \mathrm{H}_{26} \mathrm{NO}_{2}\left(\mathrm{M}^{+}+\mathrm{H}\right)$ 300.1958; found 300.1955 .

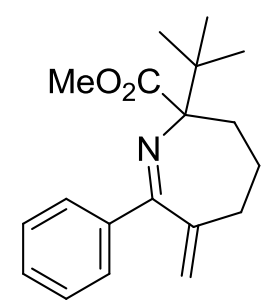

methyl 2-(tert-butyl)-6-methylene-7-phenyl-3,4,5,6-tetrahydro-2 $H$-azepine-2carboxylate (4c)

Prepared from methyl 2-(tert-butyl)-2-isocyanohept-6-enoate (45 mg, $0.2 \mathrm{mmol}$, 
1.0 equiv) and iodobenzene ( $61 \mathrm{mg}, 0.3 \mathrm{mmol}, 1.5$ equiv) according to the general procedure. Column chromatography purification (EtOAc : petroleum ether $30: 1$ ) furnished the product $\mathbf{4 c}$ as colorless solid (50 $\mathrm{mg}, 0.16 \mathrm{mmol}, 83 \%$ yield). (new compound). ${ }^{1} \mathrm{H}$ NMR (400 MHz, $\left.\mathrm{CDCl}_{3}\right)$ : $\delta$ 7.95-7.91 (m, 2H), 7.43-7.35 (m, 3H), $5.36(\mathrm{~s}, 1 \mathrm{H}), 4.78(\mathrm{~s}, 1 \mathrm{H}), 3.54(\mathrm{~s}, 3 \mathrm{H}), 2.40-2.29(\mathrm{~m}, 2 \mathrm{H}), 2.05-1.80(\mathrm{~m}, 3 \mathrm{H})$, 1.53-1.49 (m, 1H), $1.12(\mathrm{~s}, 9 \mathrm{H}) ;{ }^{13} \mathrm{C} \mathrm{NMR}\left(125 \mathrm{MHz}, \mathrm{CDCl}_{3}\right): \delta$ 173.1, 169.9, 146.4, 139.8, 129.8, 128.4, 127.9, 116.7, 74.3, 50.6, 39.9, 33.5, 28.4, 26.5, 26.1; HRMS: calcd for $\mathrm{C}_{19} \mathrm{H}_{26} \mathrm{NO}_{2}\left(\mathrm{M}^{+}+\mathrm{H}\right) 300.1958$; found 300.1958 .

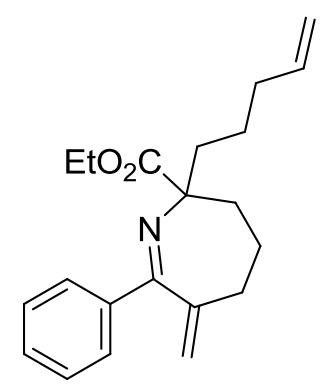

ethyl 6-methylene-2-(pent-4-en-1-yl)-7-phenyl-3,4,5,6-tetrahydro-2 $H$-azepine-2carboxylate (4d)

Prepared from ethyl 2-isocyano-2-(pent-4-en-1-yl)hept-6-enoate $(50 \mathrm{mg}, 0.2$ mmol, 1.0 equiv) and iodobenzene ( $61 \mathrm{mg}, 0.3 \mathrm{mmol}, 1.5$ equiv) according to the general procedure. Column chromatography purification (EtOAc : petroleum ether 30 : 1) furnished the product $4 \mathbf{d}$ as colorless oil $(59 \mathrm{mg}, 0.16 \mathrm{mmol}, 83 \%$ yield). (new compound). ${ }^{1} \mathrm{H}$ NMR (400 MHz, $\left.\mathrm{CDCl}_{3}\right): \delta 7.83(\mathrm{dd}, J=8.0,1.2 \mathrm{~Hz}, 2 \mathrm{H}), 7.39-7.33$ (m, 3H), 5.88-5.81 (m, 1H), $5.39(\mathrm{~s}, 1 \mathrm{H}), 5.06-4.95(\mathrm{~m}, 2 \mathrm{H}), 4.86(\mathrm{~d}, J=1.2 \mathrm{~Hz}, 1 \mathrm{H})$, 4.18-4.03 (m, 2H), 2.43-2.39 (m, 1H), 2.19-2.08 (m, 4H), 2.00-1.93 (m, 2H), $1.87-1.79(\mathrm{~m}, 3 \mathrm{H}), 1.72-1.60(\mathrm{~m}, 1 \mathrm{H}), 1.50-1.40(\mathrm{~m}, 1 \mathrm{H}), 1.22(\mathrm{t}, J=6.8 \mathrm{~Hz}, 3 \mathrm{H}) ;{ }^{13} \mathrm{C}$ NMR (125 MHz, $\left.\mathrm{CDCl}_{3}\right): \delta 173.9,171.2,146.7,140.5,138.9,129.9,128.7,127.9$, 118.7, 114.4, 69.9, 60.5, 43.3, 34.0, 34.0, 25.9, 23.6, 14.2; HRMS: calcd for $\mathrm{C}_{21} \mathrm{H}_{28} \mathrm{NO}_{2}\left(\mathrm{M}^{+}+\mathrm{H}\right)$ 326.2115; found 326.2119. 


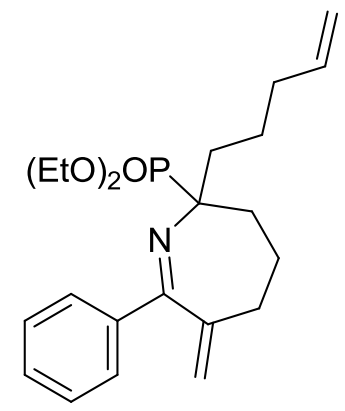

diethyl (6-methylene-2-(pent-4-en-1-yl)-7-phenyl-3,4,5,6-tetrahydro-2H-azepin-2-yl) phosphonate (4e)

Prepared from diethyl (6-isocyanoundeca-1,10-dien-6-yl)phosphonate (63 mg, $0.2 \mathrm{mmol}, 1.0$ equiv) and iodobenzene ( $61 \mathrm{mg}, 0.3 \mathrm{mmol}, 1.5$ equiv) according to the general procedure. Column chromatography purification (EtOAc : petroleum ether 15 : 1) furnished the product $4 \mathbf{e}$ as yellow oil $(63 \mathrm{mg}, 0.16 \mathrm{mmol}, 81 \%$ yield). (new compound). ${ }^{1} \mathrm{H}$ NMR (400 MHz, $\left.\mathrm{CDCl}_{3}\right): \delta 7.77(\mathrm{~d}, J=6.8 \mathrm{~Hz}, 2 \mathrm{H}), 7.37-7.30(\mathrm{~m}$, $3 \mathrm{H}), 5.78-5.74(\mathrm{~m}, 1 \mathrm{H}), 5.42(\mathrm{~s}, 1 \mathrm{H}), 5.00-4.91(\mathrm{~m}, 3 \mathrm{H}), 4.27-4.14(\mathrm{~m}, 4 \mathrm{H}), 2.55-2.52$ (m, 1H), 2.37-2.25 (m, 1H), 2.20-1.79 (m, 7H), 1.60-1.49 (m, 2H), 1.34-1.26 (m, 6H); ${ }^{13} \mathrm{C} \mathrm{NMR}\left(125 \mathrm{MHz}, \mathrm{CDCl}_{3}\right): \delta 169.8(\mathrm{~d}, J=24.3 \mathrm{~Hz}), 146.7,141.0,139.0,130.1$, 129.0, 128.1, 119.5, 114.9, 68.4 (d, $J=169.5 \mathrm{~Hz}), 63.1(\mathrm{~d}, J=7.5 \mathrm{~Hz}), 35.5,34.6$, 33.5, $31.2(\mathrm{~d}, J=153.3 \mathrm{~Hz}), 30.0,29.7,23.8(\mathrm{~d}, J=3.1 \mathrm{~Hz}), 23.2(\mathrm{~d}, J=12.4 \mathrm{~Hz})$, $16.9(\mathrm{t}, J=6.3 \mathrm{~Hz})$; HRMS: calcd for $\mathrm{C}_{22} \mathrm{H}_{33} \mathrm{NO}_{3} \mathrm{P}\left(\mathrm{M}^{+}+\mathrm{H}\right)$ 390.2193; found 390.2186.<smiles>C=C1CCC(CC(C)C)(OCC)N=C1c1ccccc1</smiles>

ethyl 2-isobutyl-5-methylene-6-phenyl-2,3,4,5-tetrahydropyridine-2-carboxylate (4f)

Prepared from ethyl 2-isobutyl-2-isocyanohex-5-enoate (45 mg, $0.2 \mathrm{mmol}, 1.0$ equiv) and iodobenzene (61 $\mathrm{mg}, 0.3 \mathrm{mmol}, 1.5$ equiv) according to the procedure but in THF as a solvent. Column chromatography purification (EtOAc : petroleum ether $30:$ 1) furnished the product $4 \mathbf{f}$ as colorless oil (46 $\mathrm{mg}, 0.15 \mathrm{mmol}, 77 \%$ yield). (new compound). ${ }^{1} \mathrm{H}$ NMR (400 MHz, $\left.\mathrm{CDCl}_{3}\right): \delta$ 7.49-7.47 (m, 2H), 7.36-7.35 (m, 3H), $5.38(\mathrm{~s}, 1 \mathrm{H}), 5.25(\mathrm{~s}, 1 \mathrm{H}), 4.23-4.12(\mathrm{~m}, 2 \mathrm{H}), 2.54-2.51(\mathrm{~m}, 2 \mathrm{H}), 2.25-2.19(\mathrm{~m}, 1 \mathrm{H})$, 1.99-1.73 (m, 4H), 1.27 (t, $J=6.8 \mathrm{~Hz}, 3 \mathrm{H}), 0.97$ (d, $J=6.4 \mathrm{~Hz}, 3 \mathrm{H}), 0.95$ (d, $J=6.4$ 
$\mathrm{Hz}, 3 \mathrm{H}) ;{ }^{13} \mathrm{C} \mathrm{NMR}\left(125 \mathrm{MHz}, \mathrm{CDCl}_{3}\right): \delta 174.3,167.3,139.8,137.6,128.9,128.7$, 127.8, 121.8, 66.9, 61.0, 48.5, 31.2, 26.4, 24.7, 24.4, 24.0, 14.2; HRMS: calcd for $\mathrm{C}_{19} \mathrm{H}_{26} \mathrm{NO}_{2}\left(\mathrm{M}^{+}+\mathrm{H}\right) 300.1958$; found 300.1960 .<smiles>C=C1CC(CC(C)C)(C(=O)OCC)N=C1c1ccccc1</smiles>

ethyl 2-isobutyl-4-methylene-5-phenyl-3,4-dihydro-2H-pyrrole-2-carboxylate (4g)

Prepared from ethyl 2-isobutyl-2-isocyanopent-4-enoate (42 mg, $0.2 \mathrm{mmol}, 1.0$ equiv) and iodobenzene (61 $\mathrm{mg}, 0.3 \mathrm{mmol}, 1.5$ equiv) according to the general procedure. Column chromatography purification (EtOAc : petroleum ether $30: 1$ ) furnished the product $\mathbf{4 g}$ as colorless oil (36 $\mathrm{mg}, 0.12 \mathrm{mmol}, 63 \%$ yield). (new compound). ${ }^{1} \mathrm{H}$ NMR (400 MHz, $\left.\mathrm{CDCl}_{3}\right)$ : $\delta$ 7.66-7.63 (m, 2H), 7.47-7.40 (m, 3H), $5.47(\mathrm{t}, J=2.4 \mathrm{~Hz}, 1 \mathrm{H}), 5.42(\mathrm{t}, J=2.4 \mathrm{~Hz}, 1 \mathrm{H}), 4.29-4.17(\mathrm{~m}, 2 \mathrm{H}), 3.27(\mathrm{dt}, J=16.8$, $2.4 \mathrm{~Hz}, 1 \mathrm{H}), 2.75$ (dt, $J=16.8,2.4 \mathrm{~Hz}, 1 \mathrm{H}), 1.99-1.93(\mathrm{~m}, 1 \mathrm{H}), 1.88-1.79(\mathrm{~m}, 2 \mathrm{H})$, $1.30(\mathrm{t}, J=6.8 \mathrm{~Hz}, 3 \mathrm{H}), 0.96(\mathrm{~d}, J=6.0 \mathrm{~Hz}, 6 \mathrm{H}) ;{ }^{13} \mathrm{C} \mathrm{NMR}\left(125 \mathrm{MHz}, \mathrm{CDCl}_{3}\right): \delta$ 174.0, 172.3, 148.1, 133.4, 130.0, 128.8, 128.3, 112.6, 79.3, 61.2, 47.7, 40.6, 25.1, 24.1, 23.8, 14.2; HRMS: calcd for $\mathrm{C}_{18} \mathrm{H}_{24} \mathrm{NO}_{2}\left(\mathrm{M}^{+}+\mathrm{H}\right)$ 286.1802; found 286.1804.<smiles>C=C1CC(C(=O)OCC)N=C1c1ccccc1</smiles>

ethyl 4-methylene-5-phenyl-3,4-dihydro-2H-pyrrole-2-carboxylate (4h)

Prepared from ethyl 2-isocyanopent-4-enoate (30 mg, $0.2 \mathrm{mmol}, 1.0$ equiv) and iodobenzene (61 mg, $0.3 \mathrm{mmol}, 1.5$ equiv) according to the general procedure. Column chromatography purification (EtOAc : petroleum ether $30: 1$ ) furnished the product $4 \mathrm{~h}$ as yellow oil (33 $\mathrm{mg}, 0.14 \mathrm{mmol}, 72 \%$ yield). (new compound). ${ }^{1} \mathrm{H}$ NMR (400 MHz, $\left.\mathrm{CDCl}_{3}\right)$ : 7.67-7.65 (m, 2H), 7.46-7.40 (m, 3H), 5.51-5.47 (m, 2H), $4.87(\mathrm{t}$, $J=6.4 \mathrm{~Hz}, 1 \mathrm{H}), 4.30-4.22(\mathrm{~m}, 2 \mathrm{H}), 3.06-3.03(\mathrm{~m}, 2 \mathrm{H}), 1.32(\mathrm{t}, J=6.8 \mathrm{~Hz}, 3 \mathrm{H}) ;{ }^{13} \mathrm{C}$ NMR (125 MHz, $\left.\mathrm{CDCl}_{3}\right): \delta 174.8,172.0,147.8,133.1,130.2,128.7,128.4,112.9$, 71.0, 61.4, 34.3, 14.2; HRMS: calcd for $\mathrm{C}_{14} \mathrm{H}_{16} \mathrm{NO}_{2}\left(\mathrm{M}^{+}+\mathrm{H}\right)$ 230.1176; found 230.1174 . 
<smiles>C=C(C)/C(=C/c1ccccc1)CC(CC)(CC(C)C)OCC</smiles>

(E)-ethyl 4-benzylidene-2-isobutyl-5-phenyl-3,4-dihydro-2H-pyrrole-2-carboxylate (4i)

Prepared from (E)-ethyl 2-isobutyl-2-isocyano-5-phenylpent-4-enoate (57 mg, $0.2 \mathrm{mmol}, 1.0$ equiv) and iodobenzene ( $61 \mathrm{mg}, 0.3 \mathrm{mmol}, 1.5$ equiv) according to the general procedure. Column chromatography purification (EtOAc : petroleum ether 30 : 1) furnished the product $4 \mathbf{i}$ as yellow oil (70 $\mathrm{mg}, 0.19 \mathrm{mmol}, 97 \%$ yield). (new compound). ${ }^{1} \mathrm{H}$ NMR (400 MHz, $\left.\mathrm{CDCl}_{3}\right)$ : 7.66-7.65 (m, $\left.2 \mathrm{H}\right), 7.48-7.37(\mathrm{~m}, 7 \mathrm{H})$, 7.32-7.25 (m, 1H), $6.80(\mathrm{t}, J=2.4 \mathrm{~Hz}, 1 \mathrm{H}), 4.31-4.18(\mathrm{~m}, 2 \mathrm{H}), 3.55(\mathrm{dd}, J=17.2,2.8$ Hz, 1H), 3.02 (dd, $J=17.2,2.8 \mathrm{~Hz}, 1 \mathrm{H}), 1.97-1.93$ (m, 2H), 1.91-1.82 (m, 1H), 1.31 $(\mathrm{t}, J=6.8 \mathrm{~Hz}, 3 \mathrm{H}), 0.98-0.95(\mathrm{~m}, 6 \mathrm{H}) ;{ }^{13} \mathrm{C} \mathrm{NMR}\left(125 \mathrm{MHz}, \mathrm{CDCl}_{3}\right): \delta 174.5,174.5$, $140.9,136.8,134.2,130.1,129.7,129.4,129.3,129.0,128.7,128.6,81.2$, 61.7, 48.4, 27.4, 25.4, 24.4, 24.2, 14.5; HRMS: calcd for $\mathrm{C}_{24} \mathrm{H}_{28} \mathrm{NO}_{2}\left(\mathrm{M}^{+}+\mathrm{H}\right)$ 362.2115; found 362.2118 .

\section{References}

(1) Casabona, D.; Jiménez, A.; Cativiela C. Tetrahedron. 2007, 63, 5056.

(2) Gulevich, A. V.; Zhdanko, A. G.; Orru, R. V. A.; Nenajdenko, V. G. Chem. Rev. 2010, 110, 5235 .

(3) Ito, Y.; Higuchi, N.; Murakami, M. Tetrahedron Lett. 1988, 29, 5151.

(4) Banfi, L.; Basso, A.; Guanti, G.; Riva, R. Tetrahedron Lett. 2003, 44, 7655. 
VI. Copies of ${ }^{1} \mathrm{H}$ and ${ }^{13} \mathrm{C}$ NMR Spectra

$1 a$

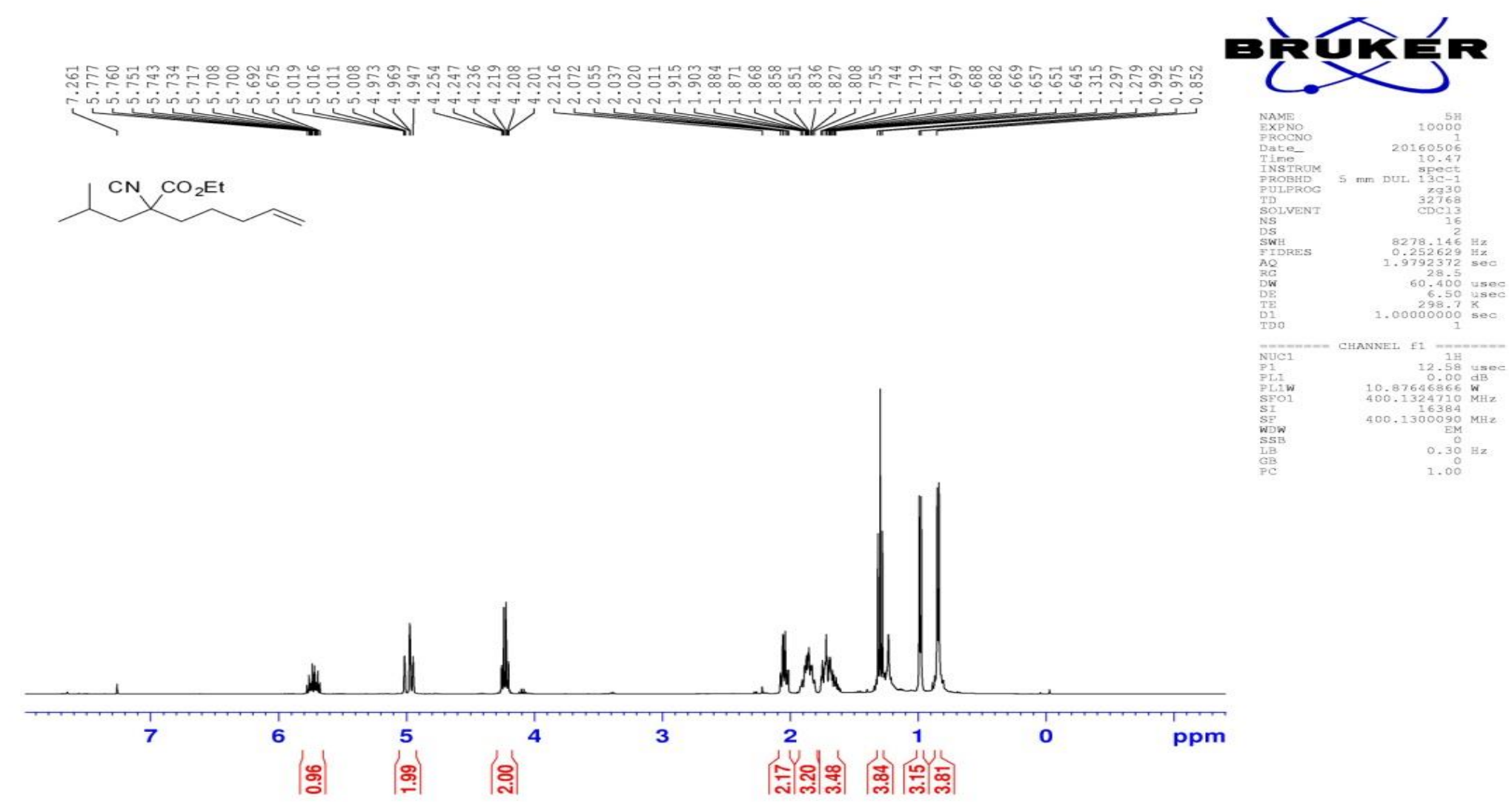




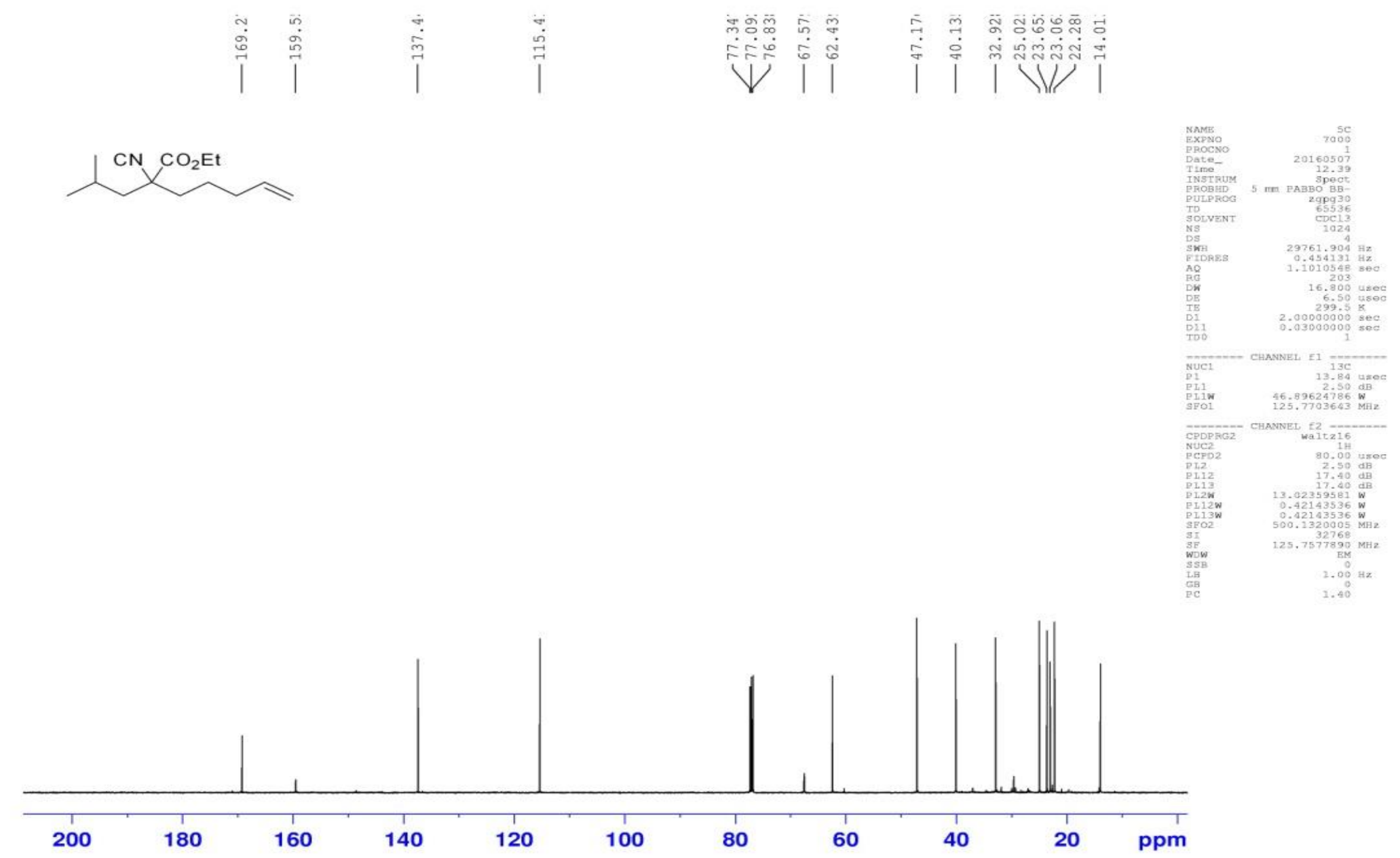




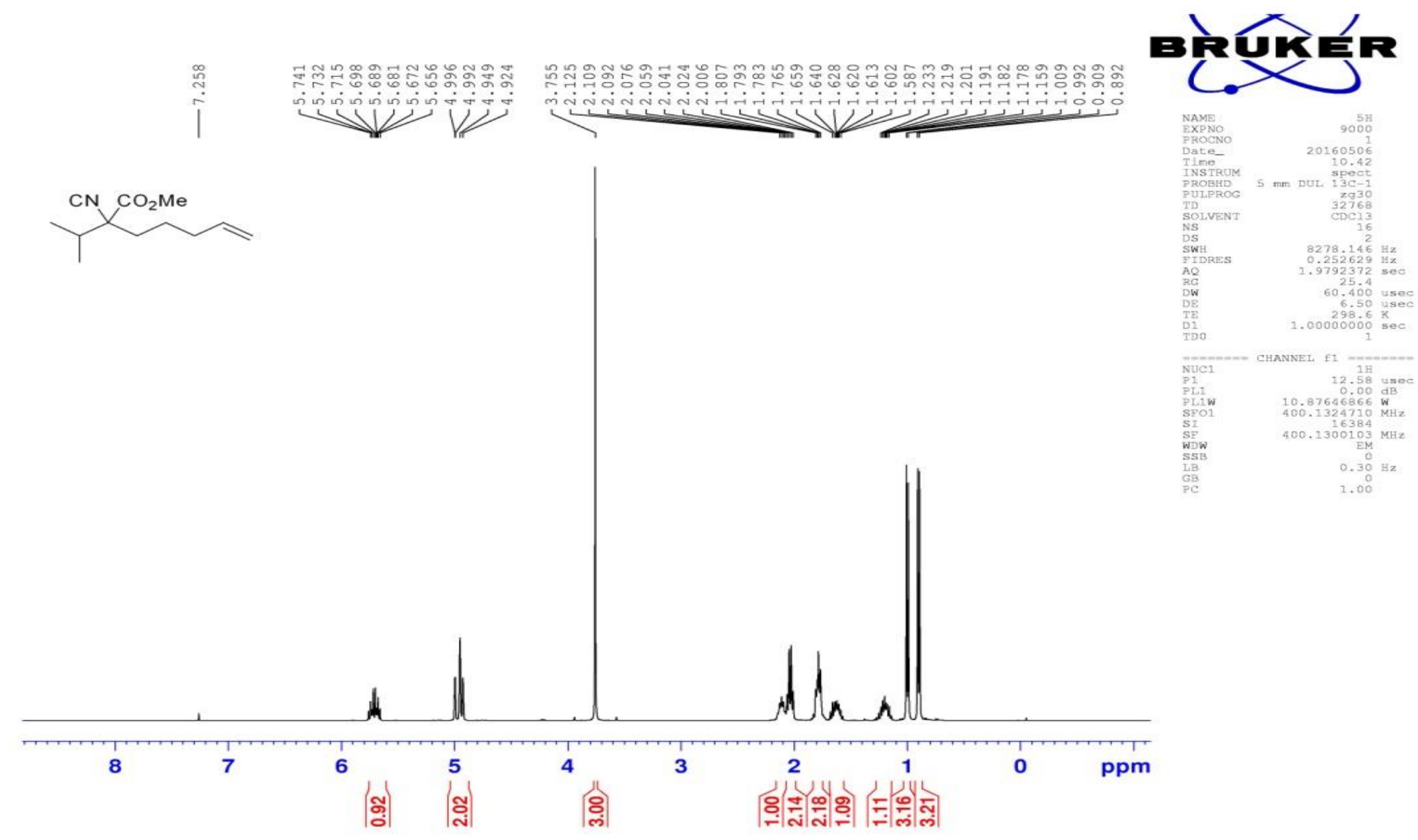




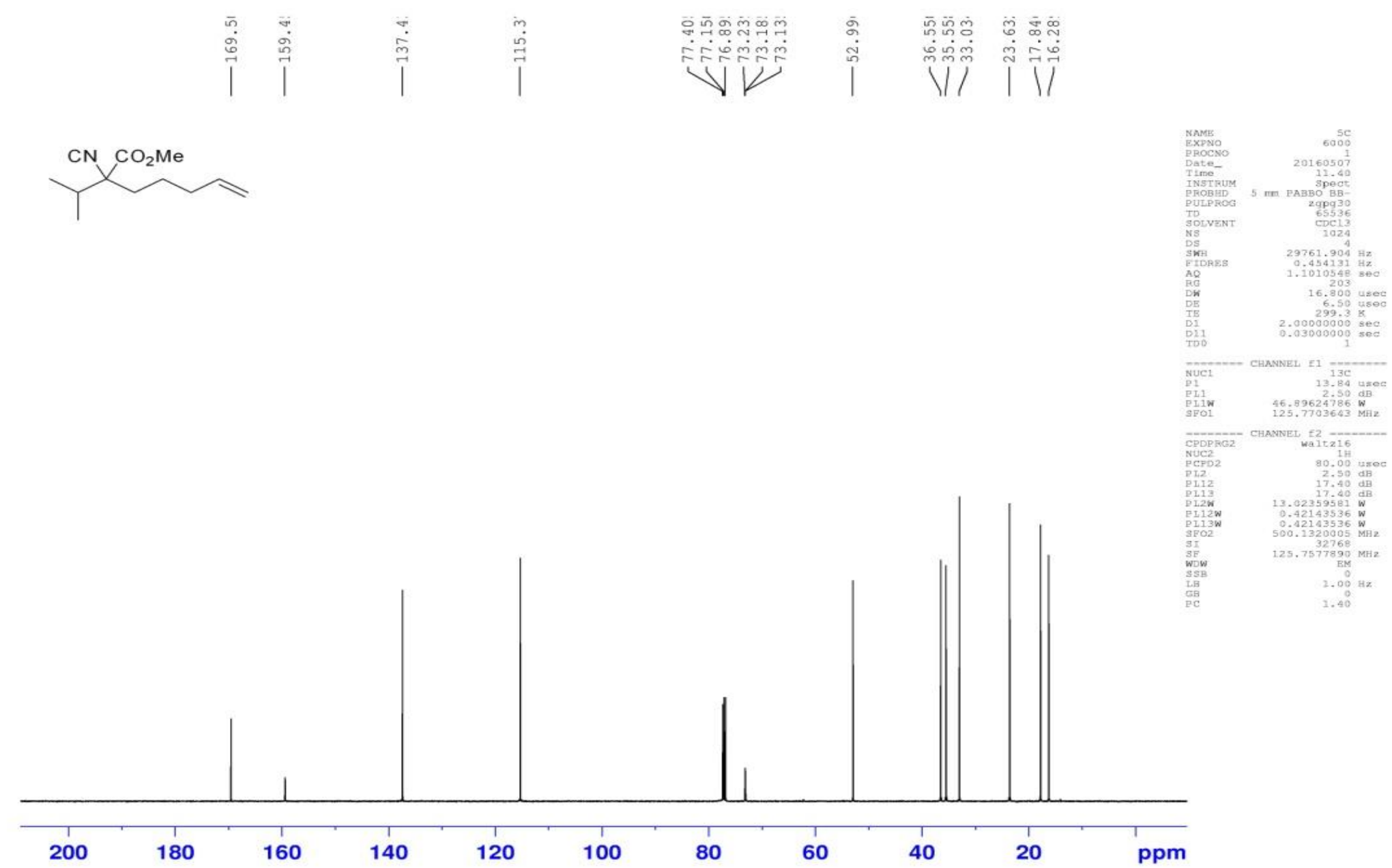




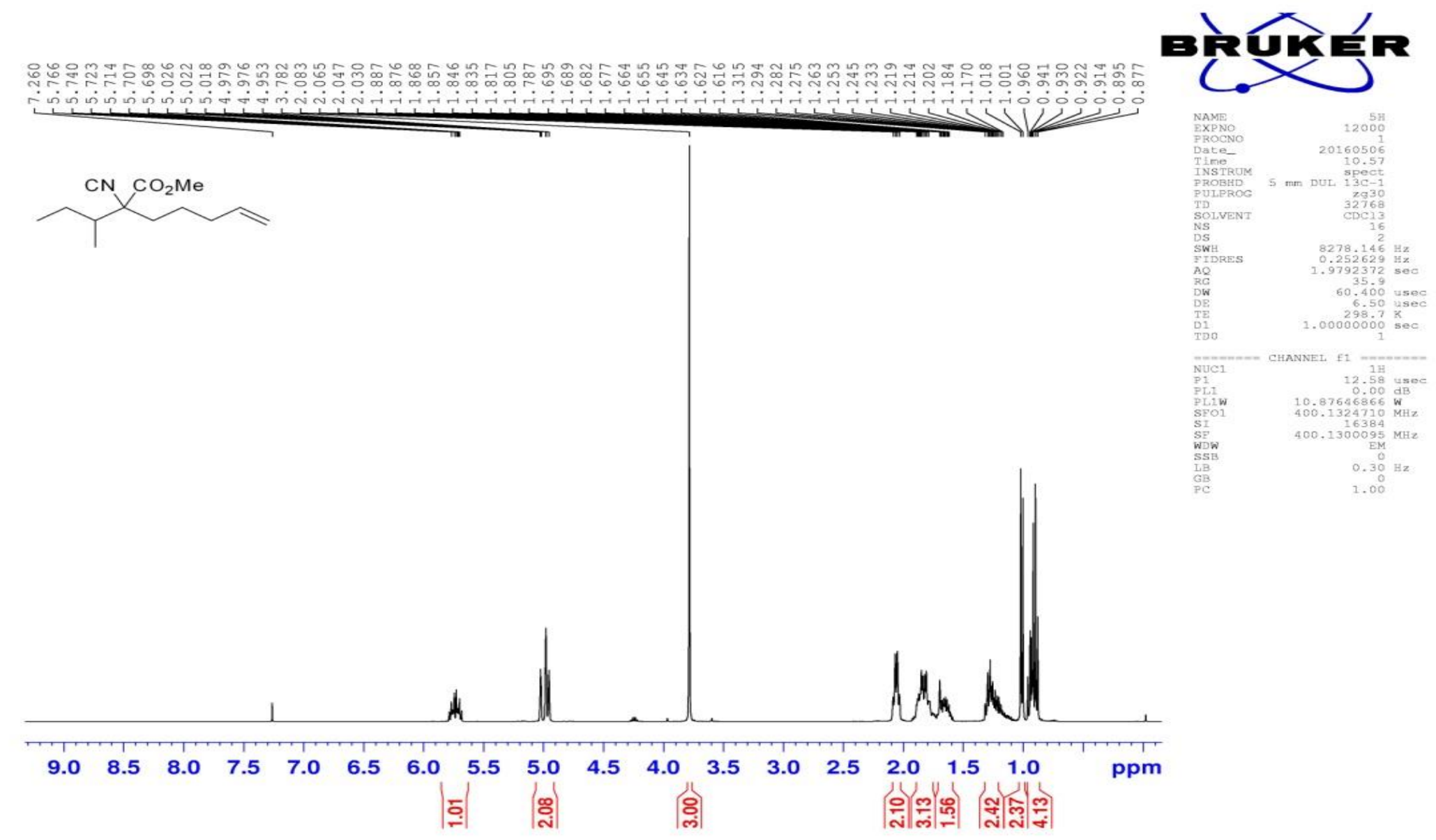




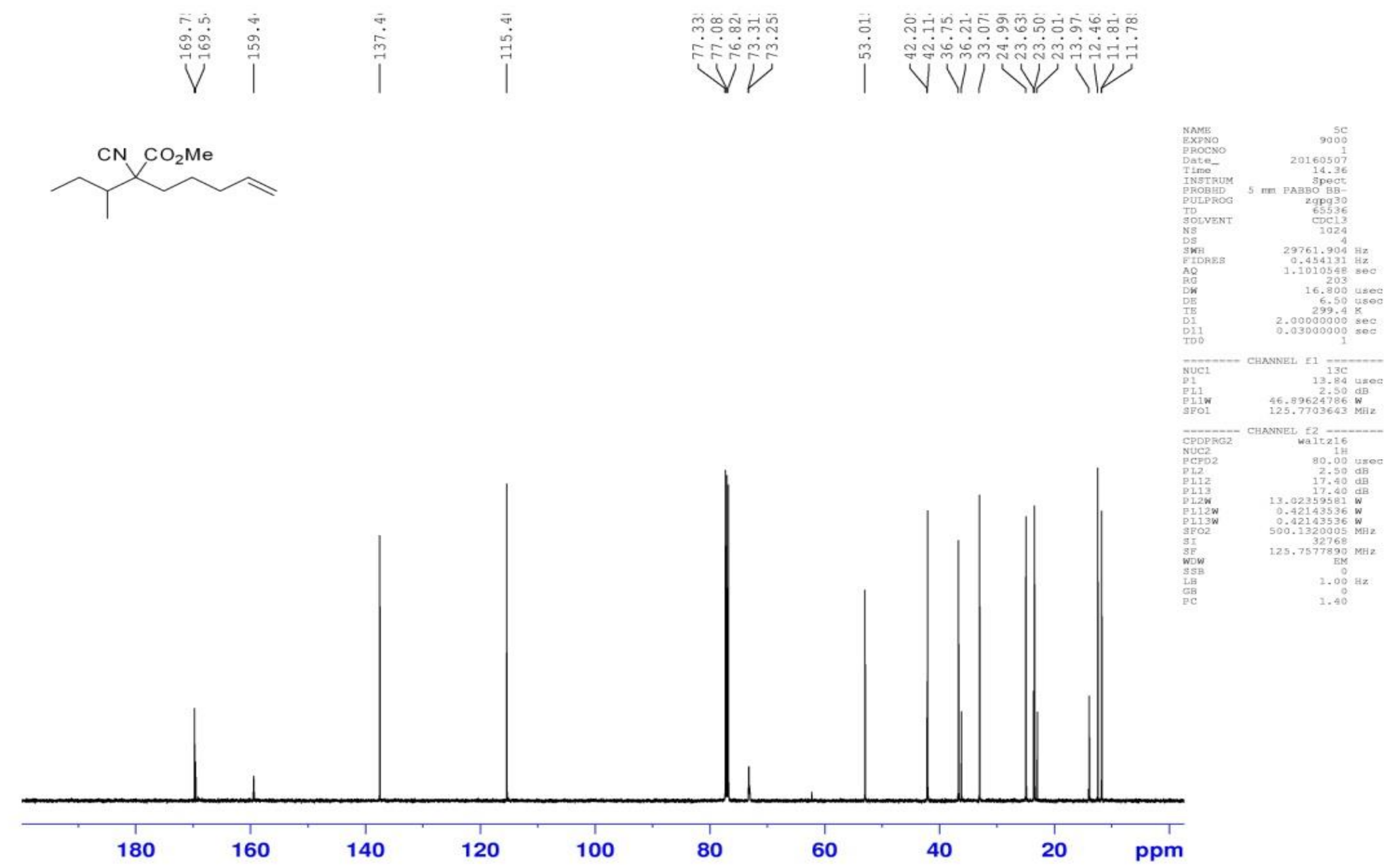




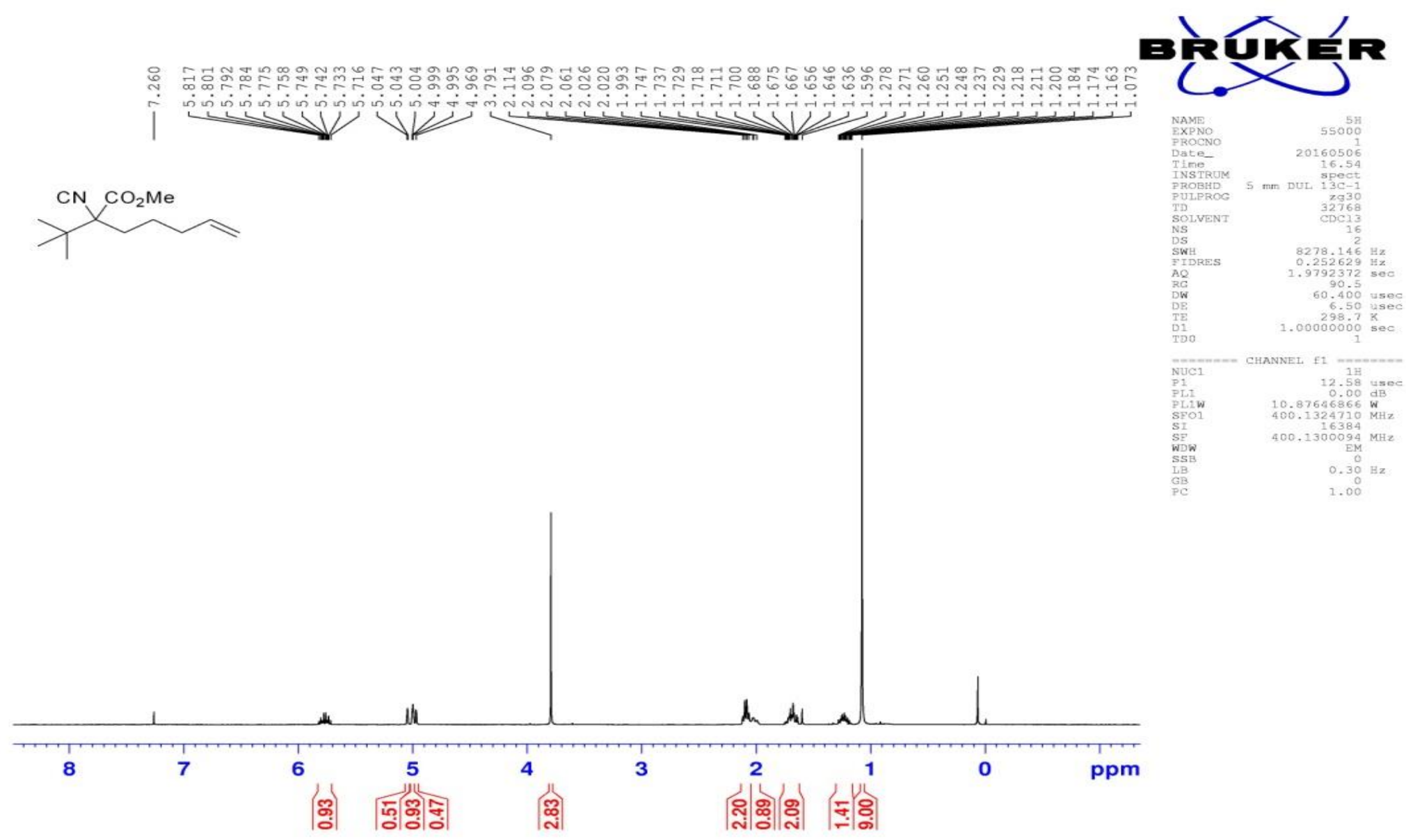




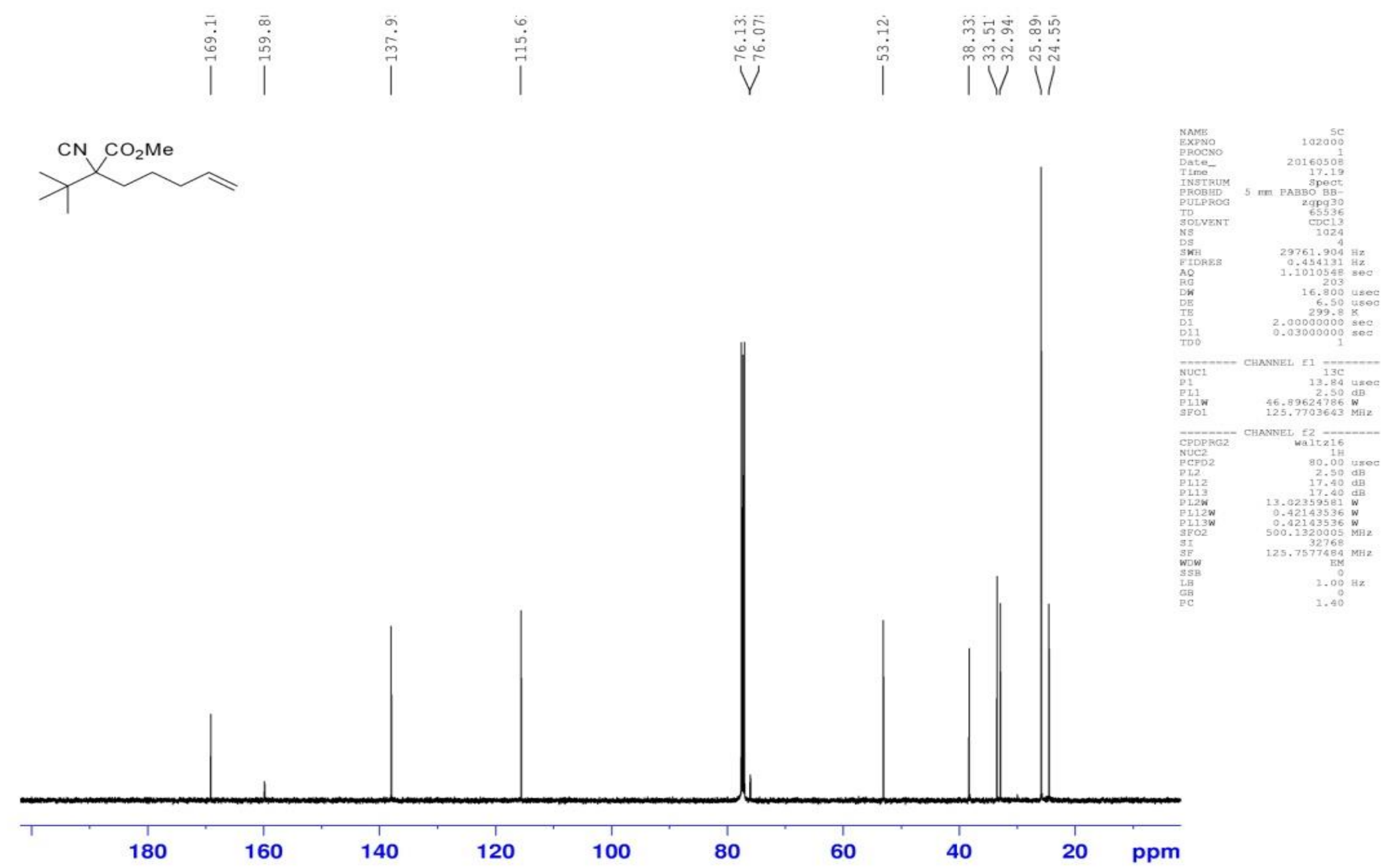



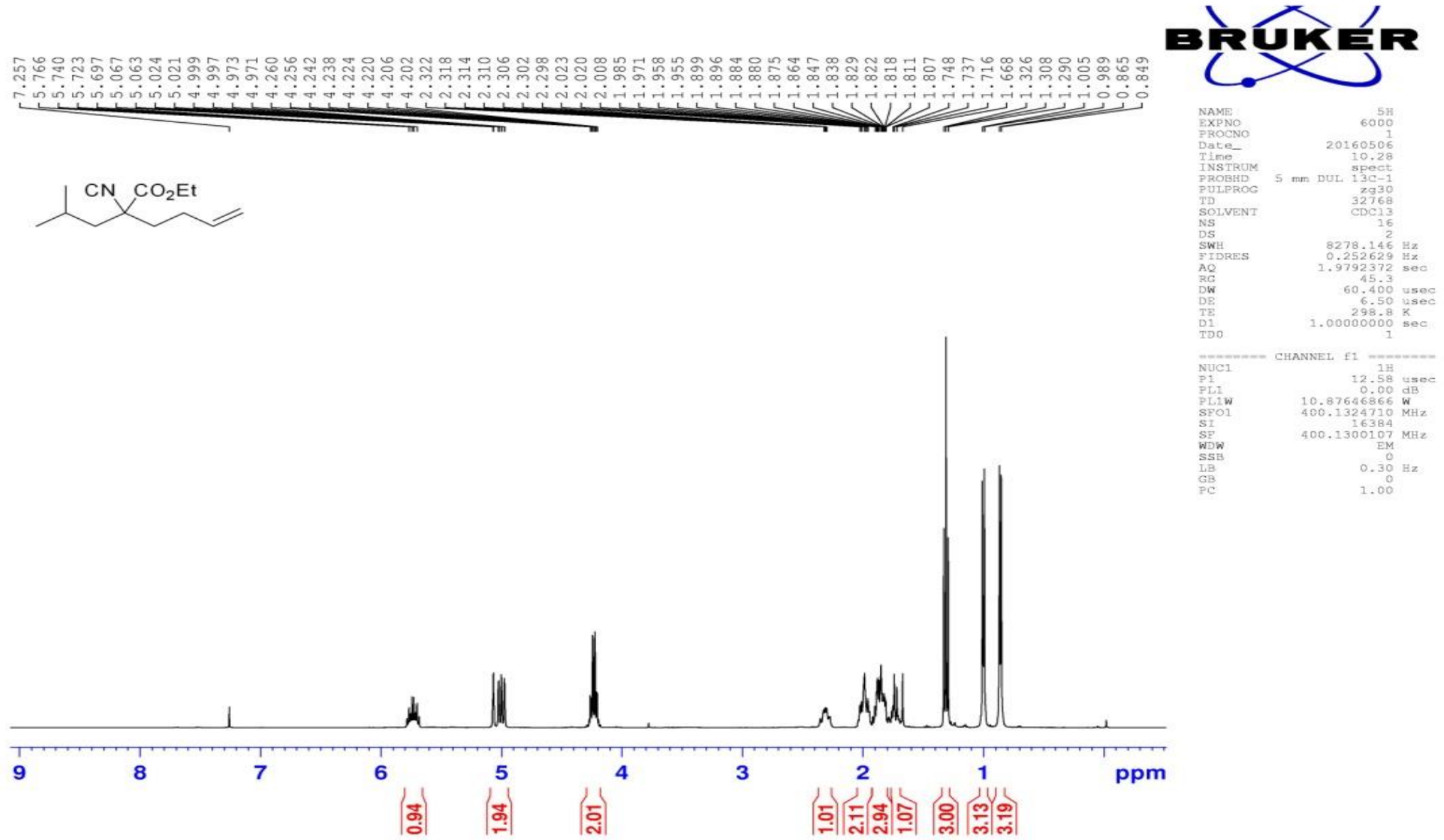


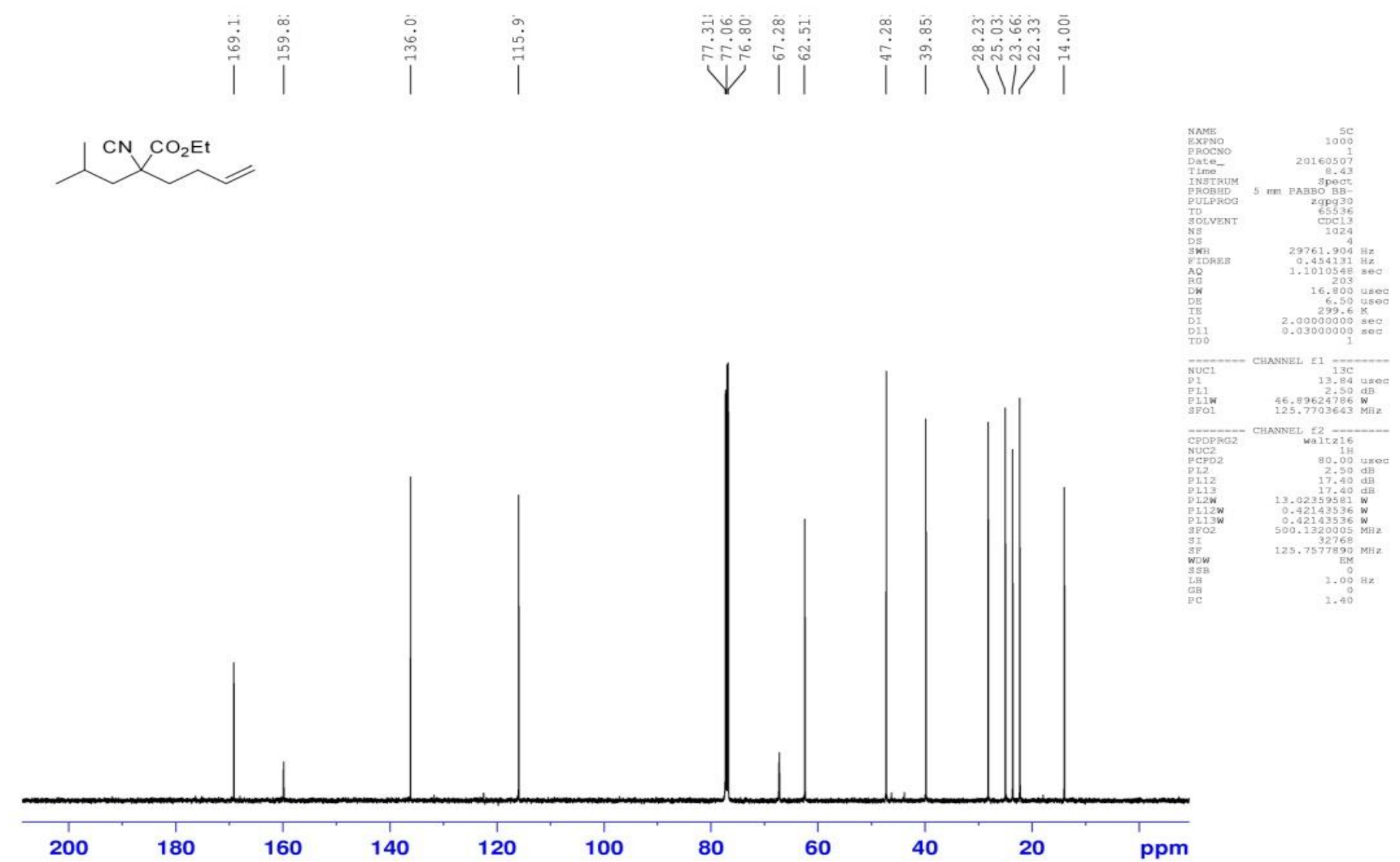




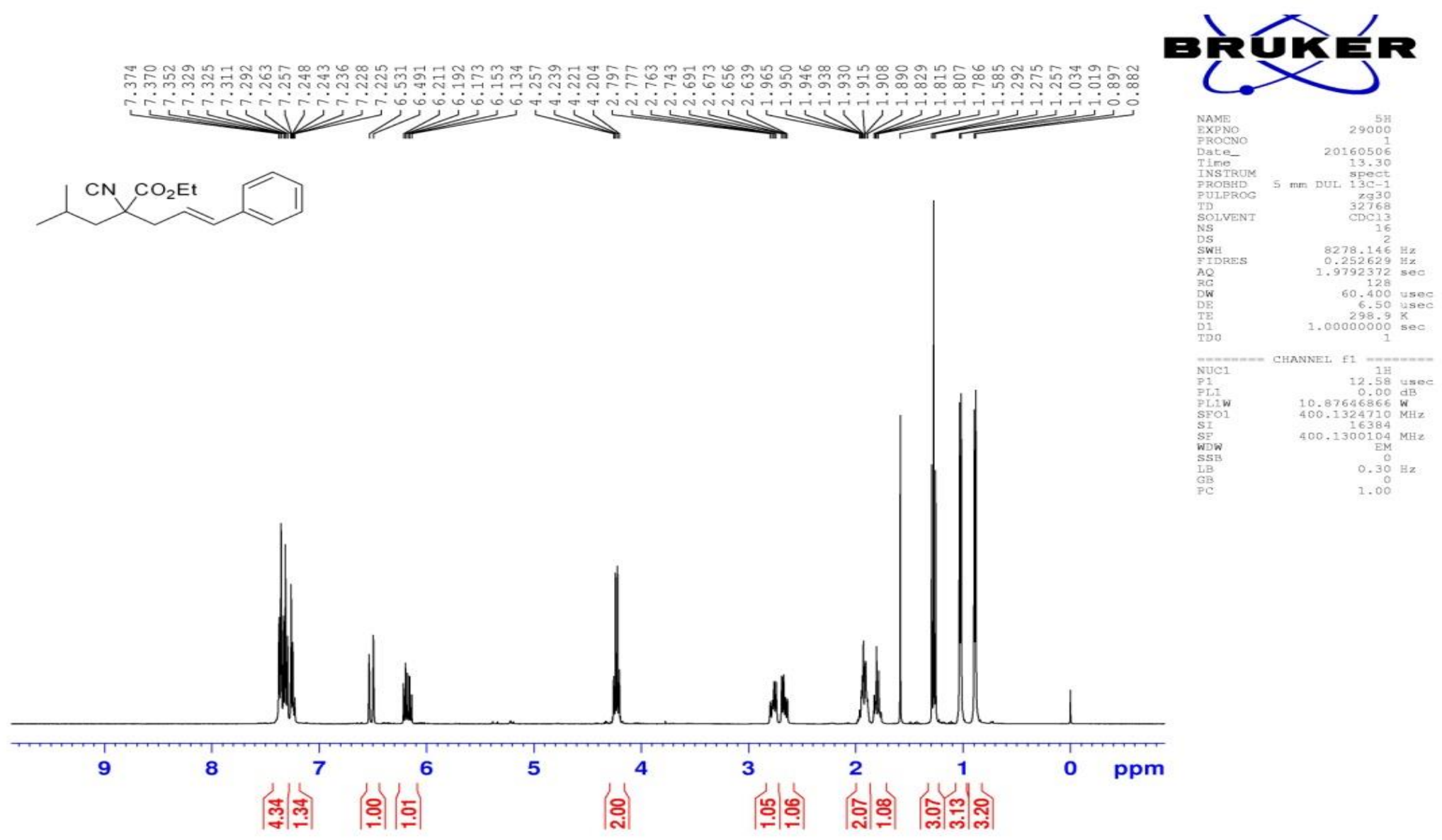




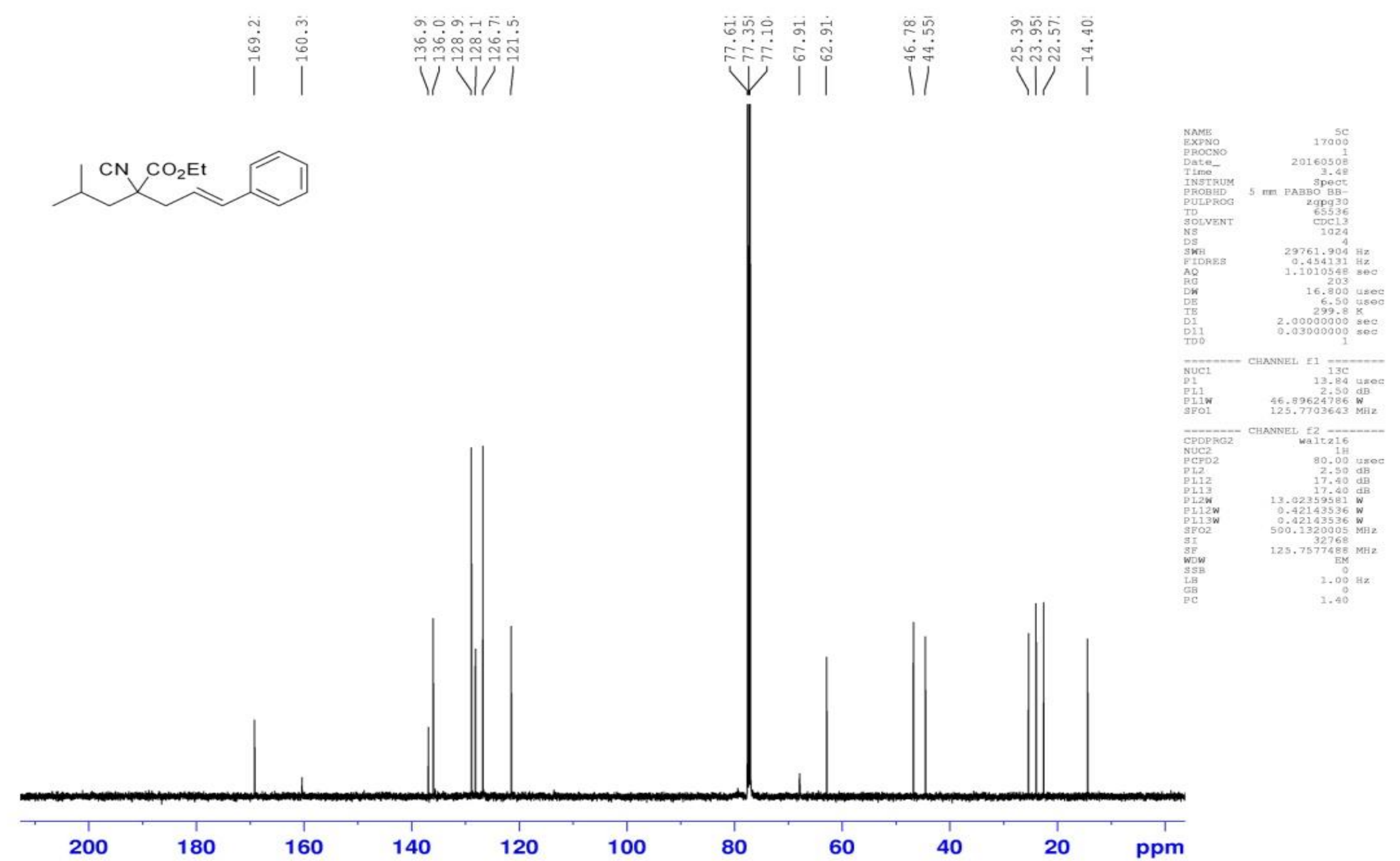




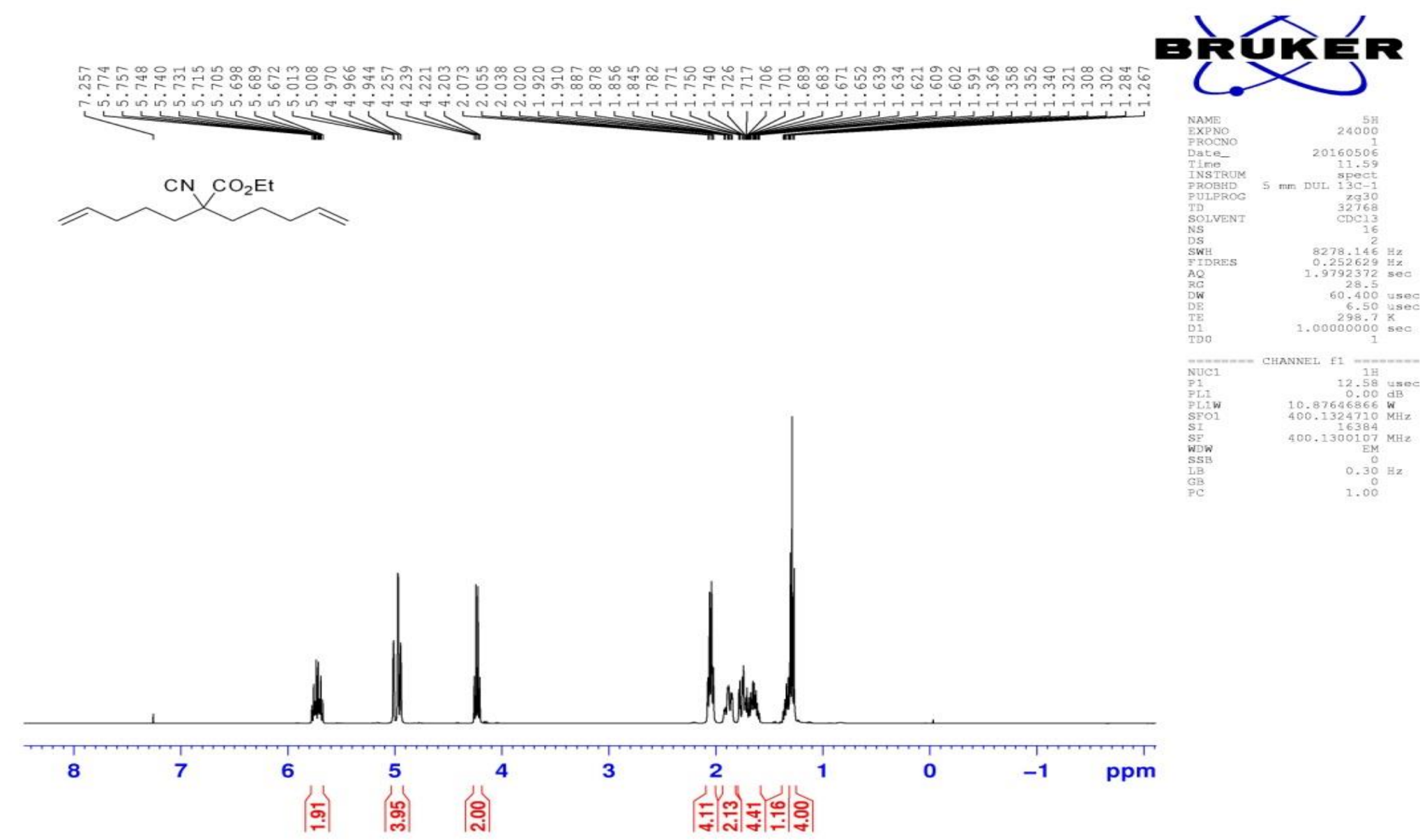




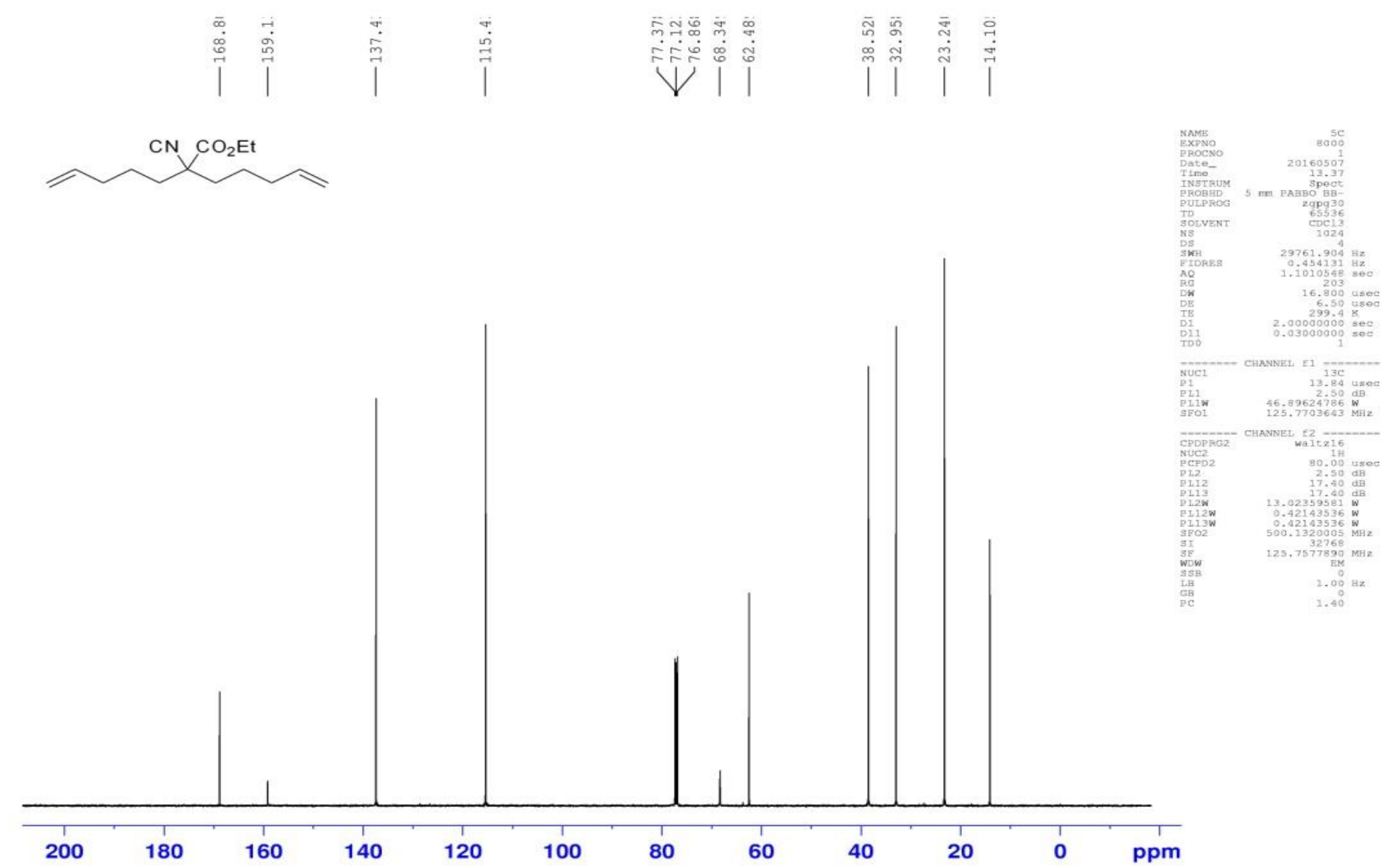




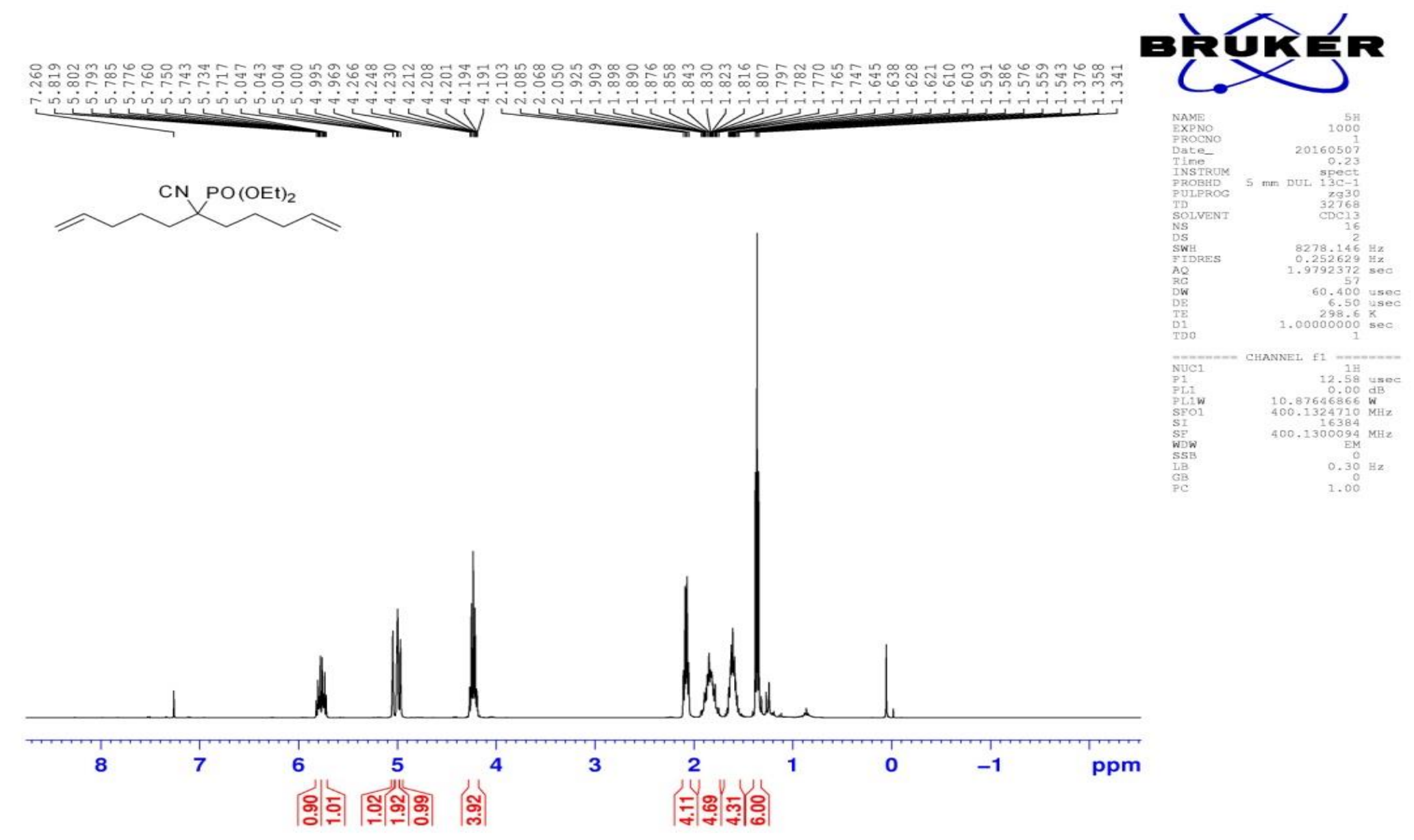




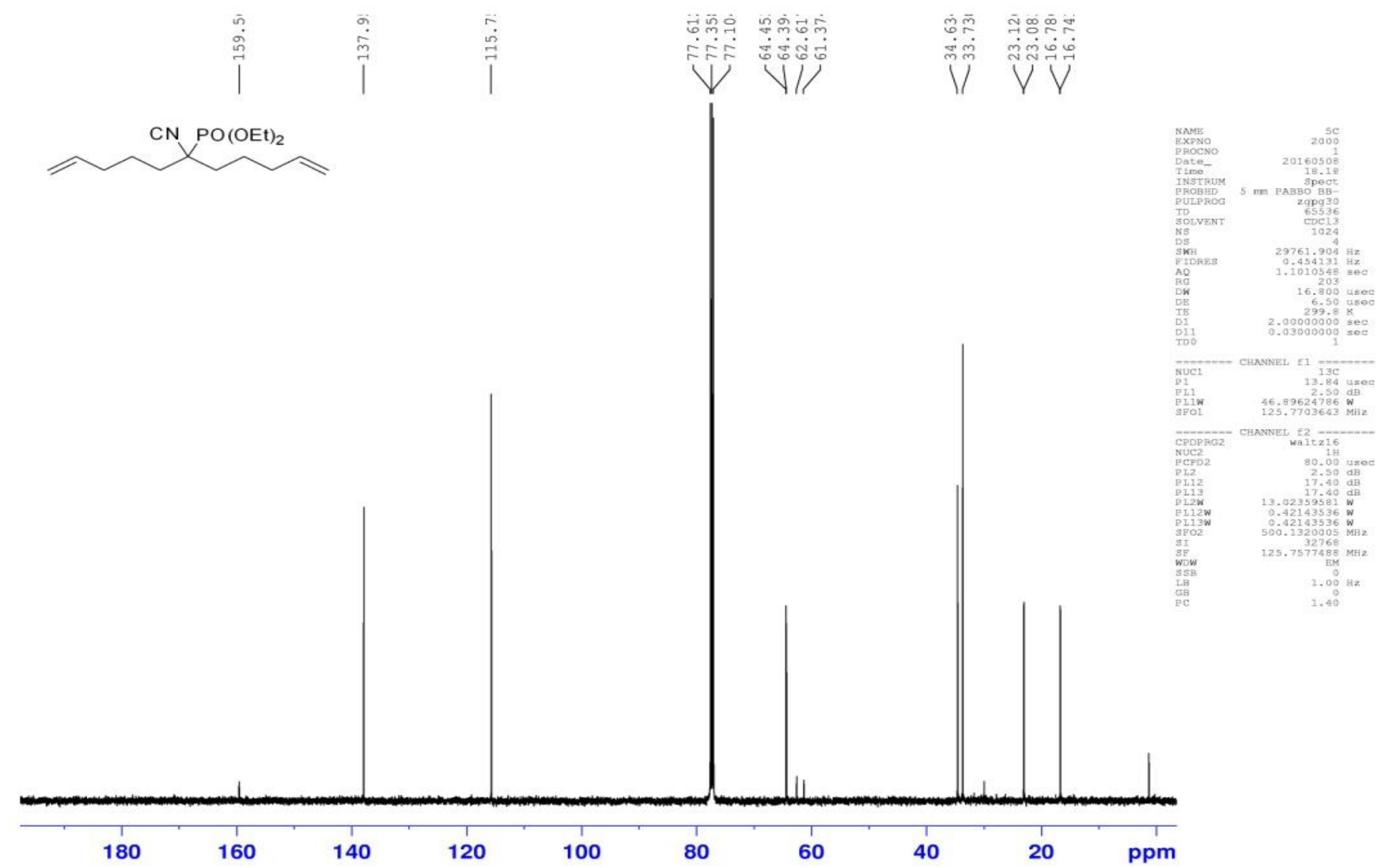




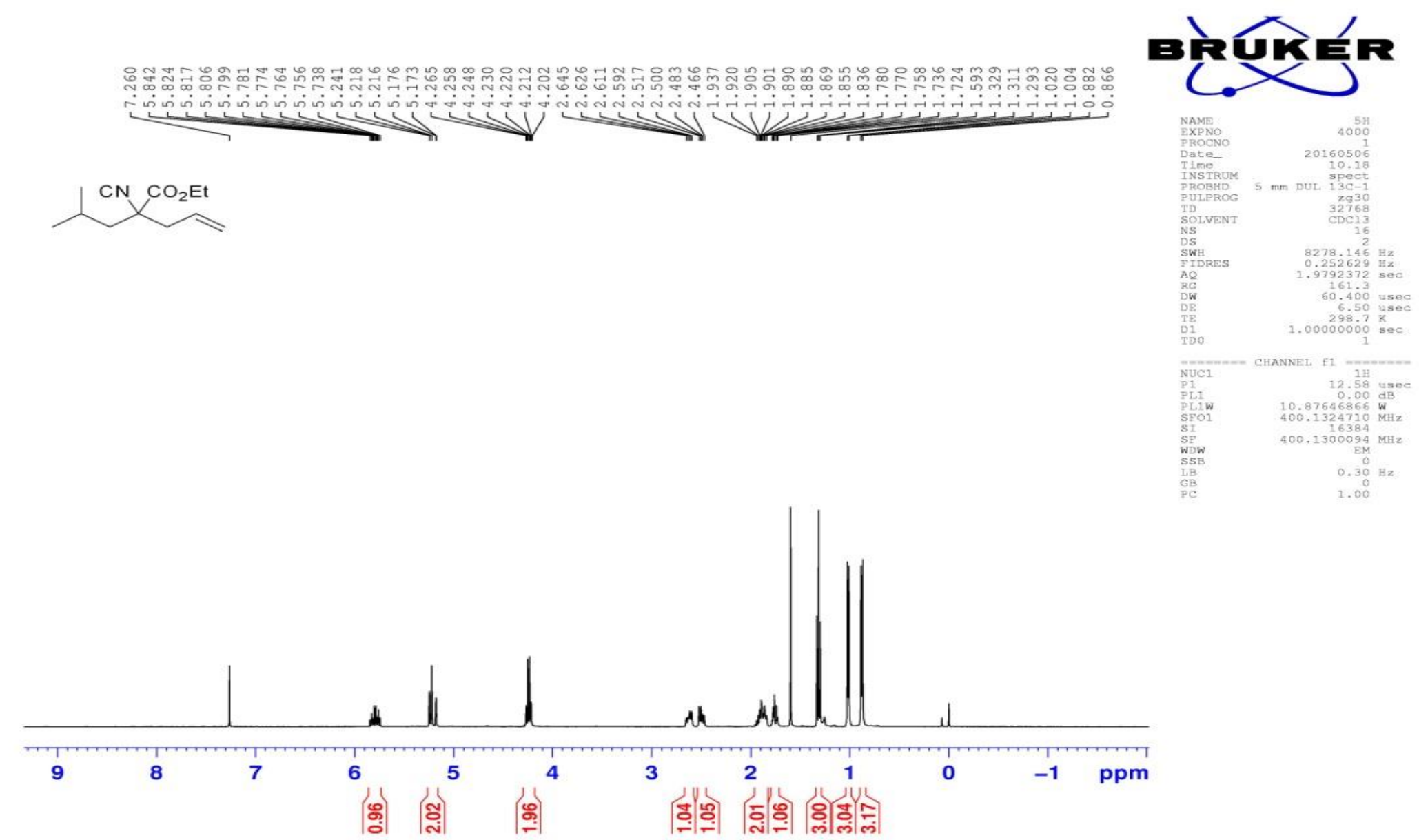




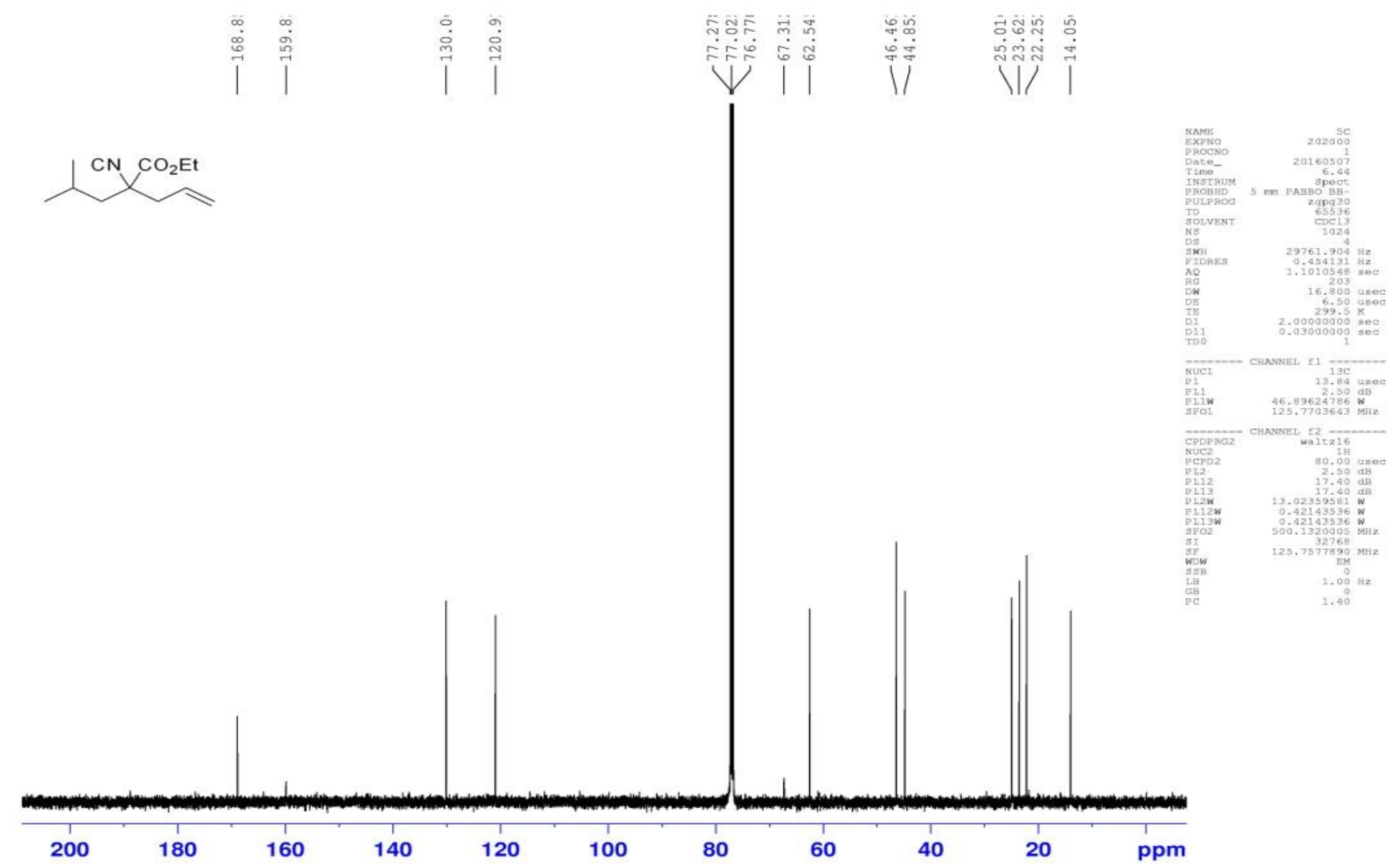




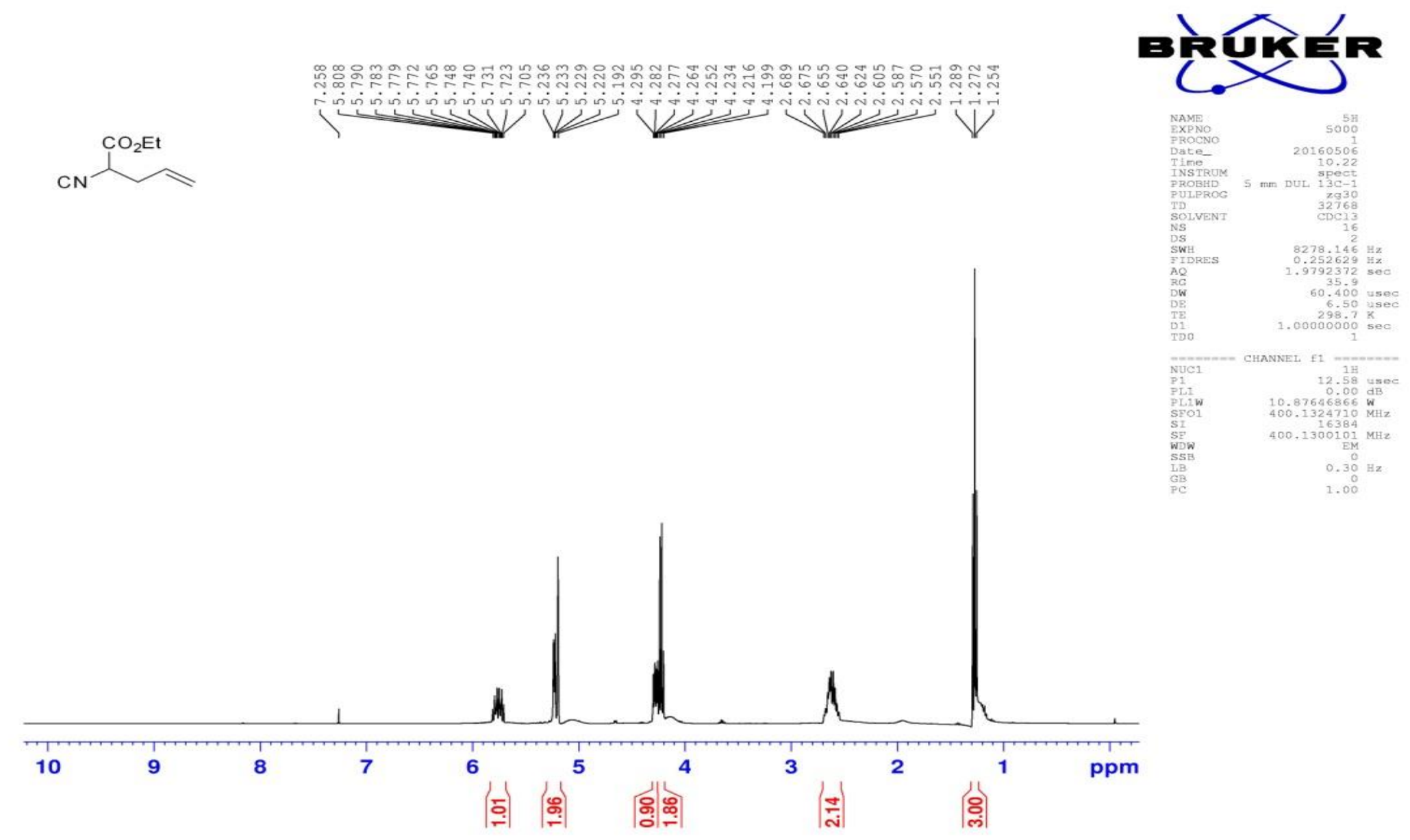




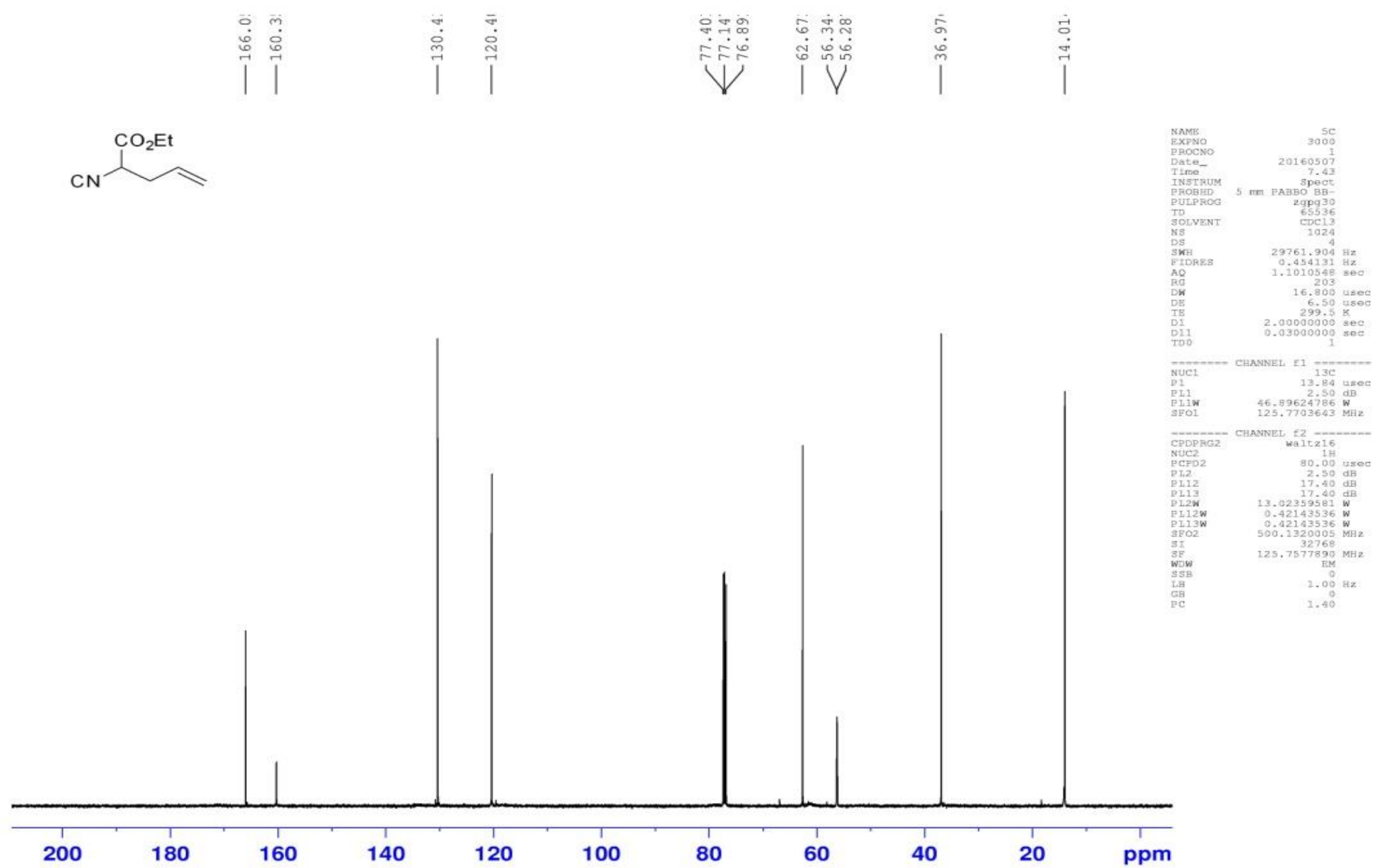




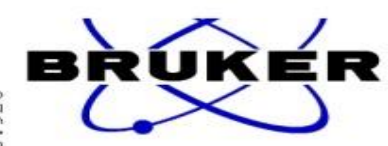

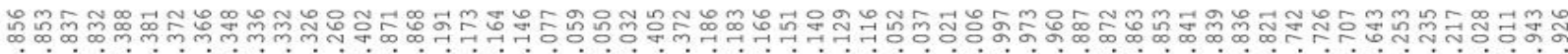
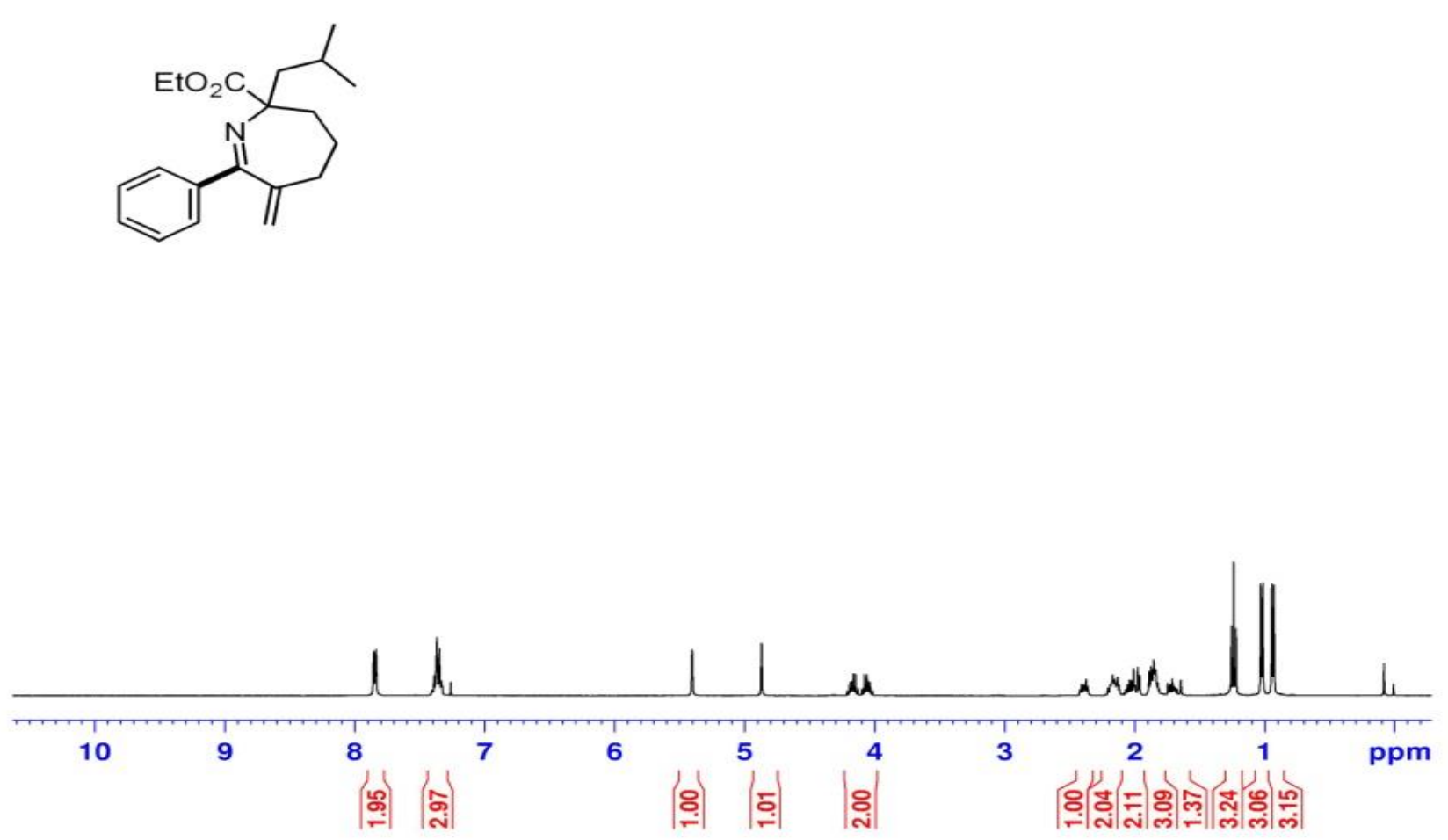


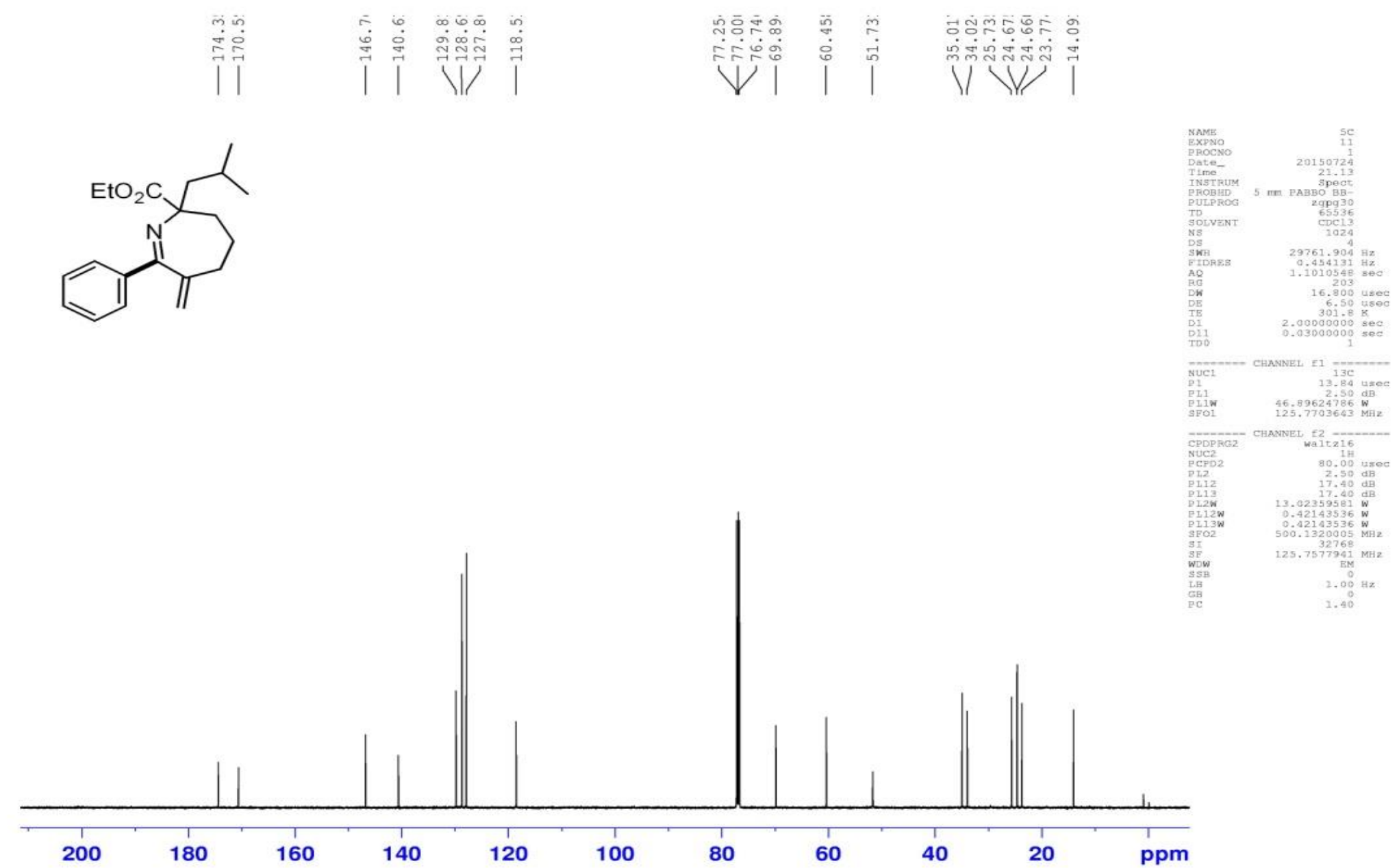


3a-HMBC

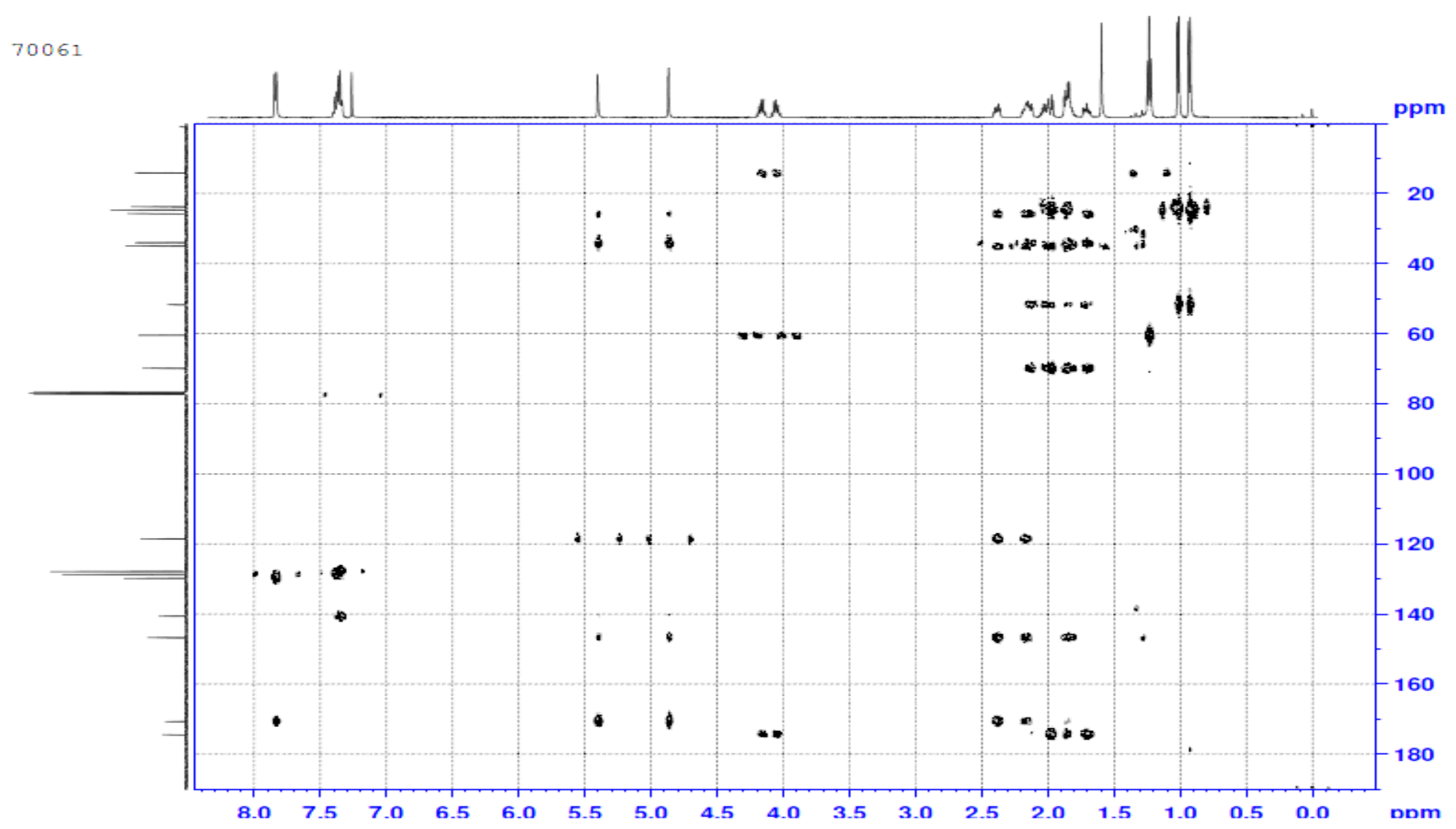


3a-HSQC

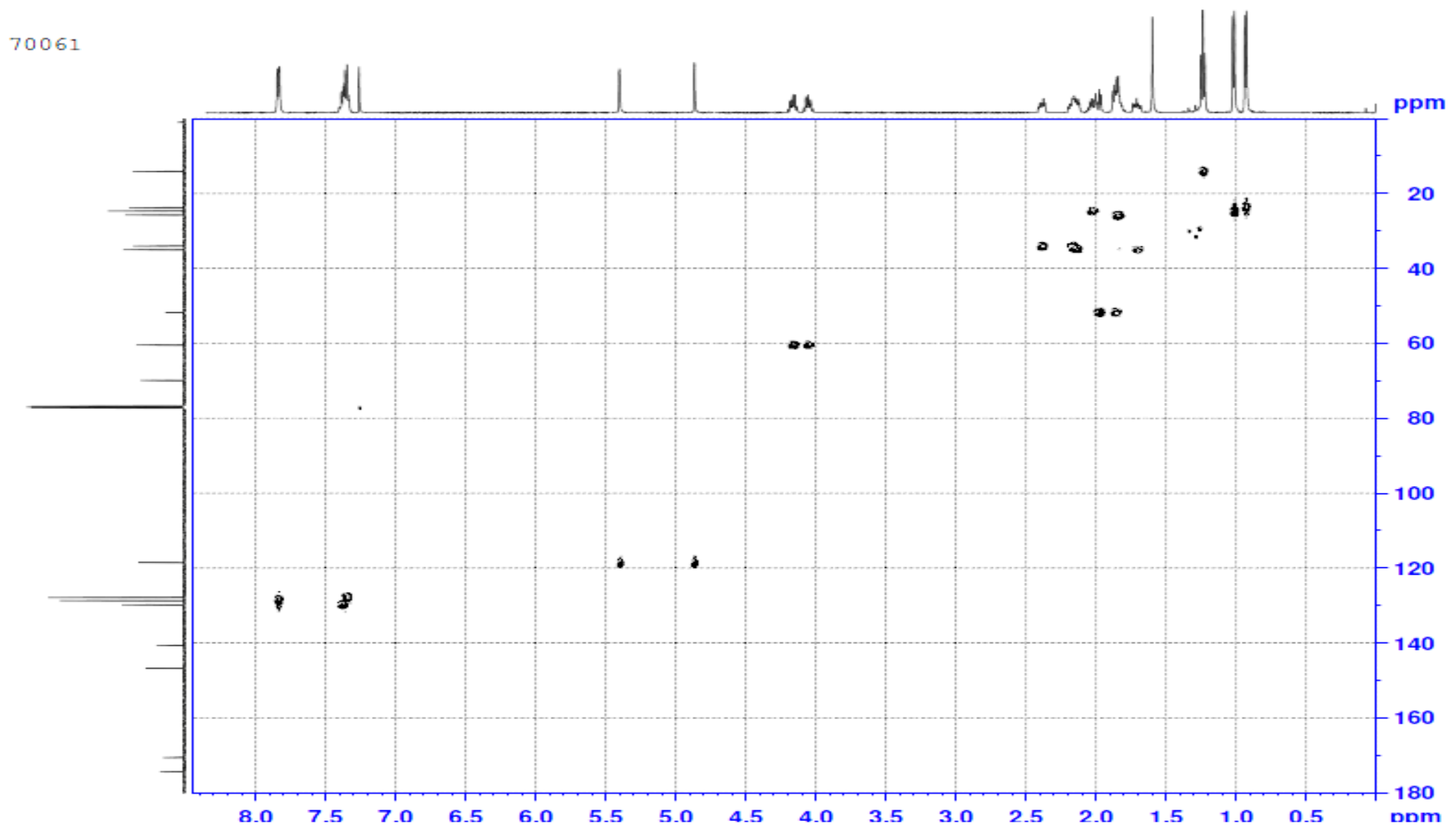


3a-COSY

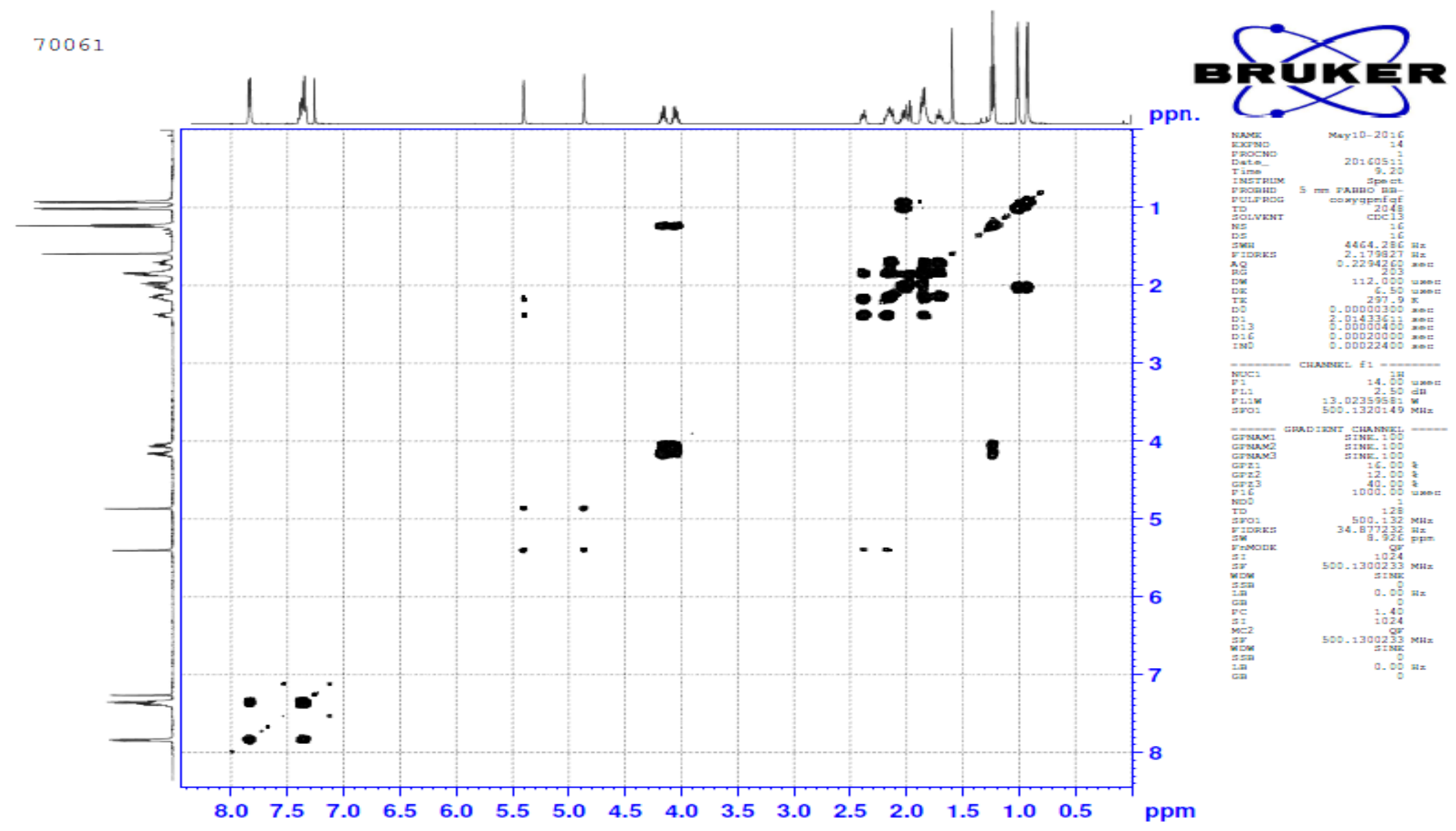




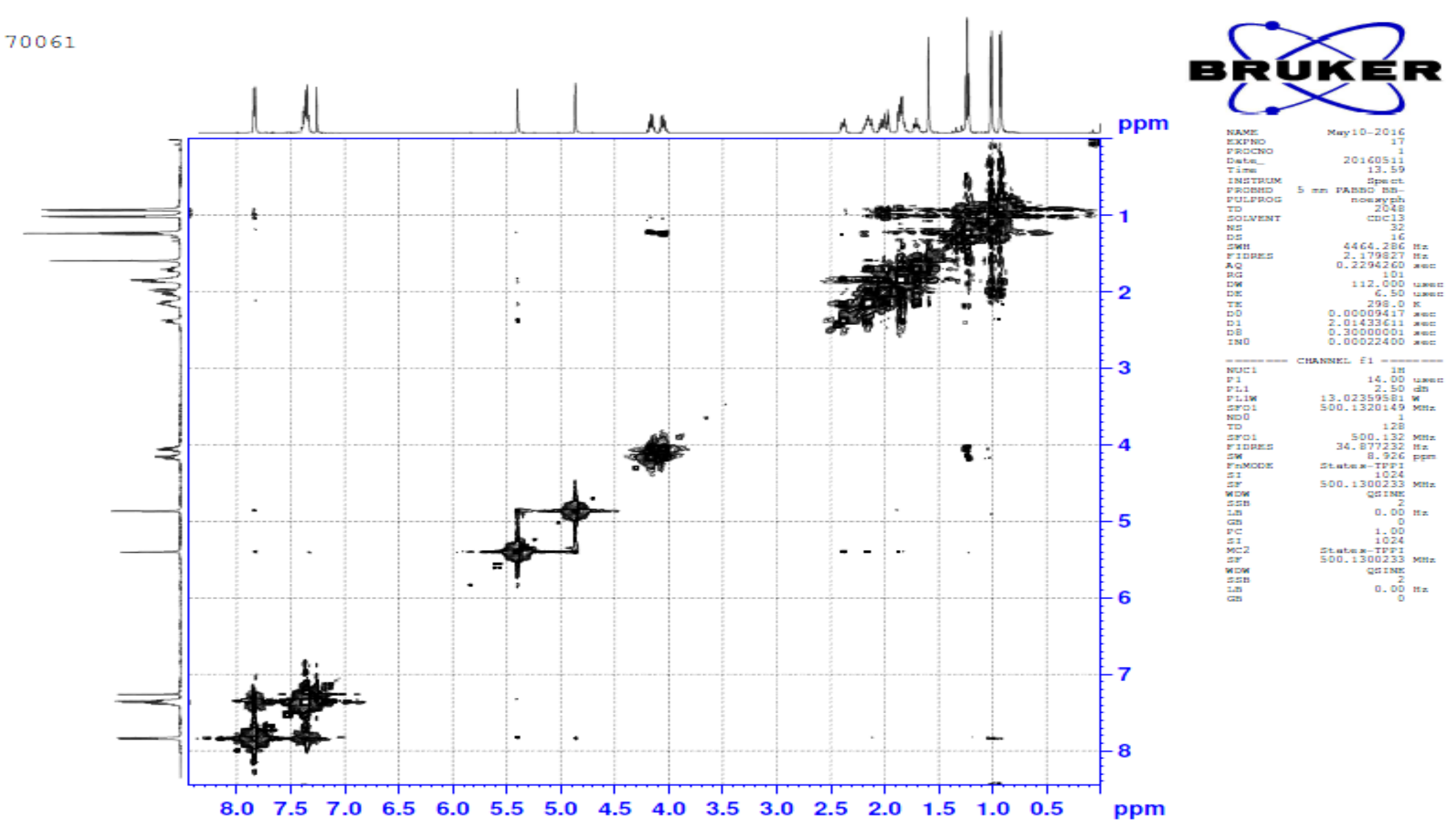




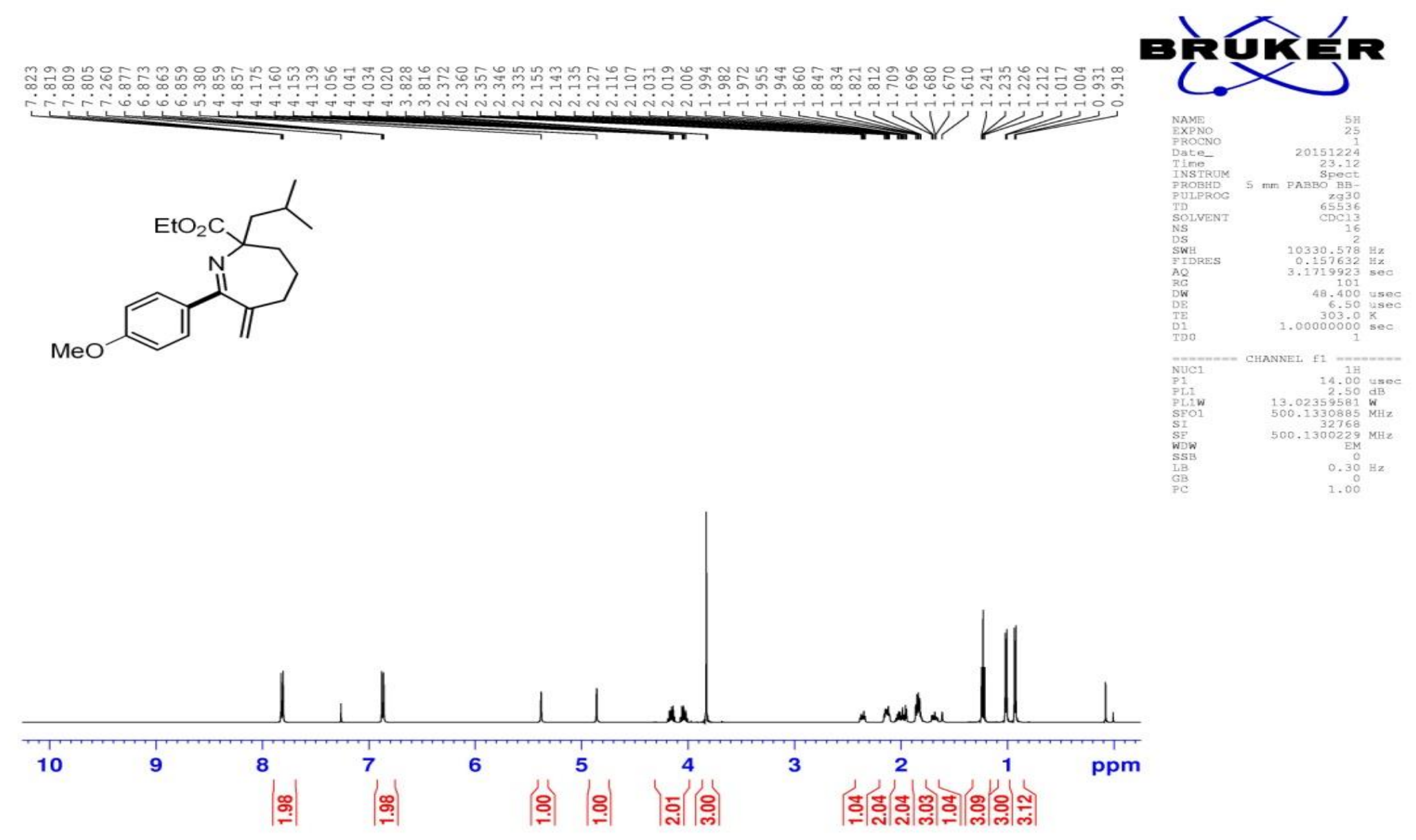




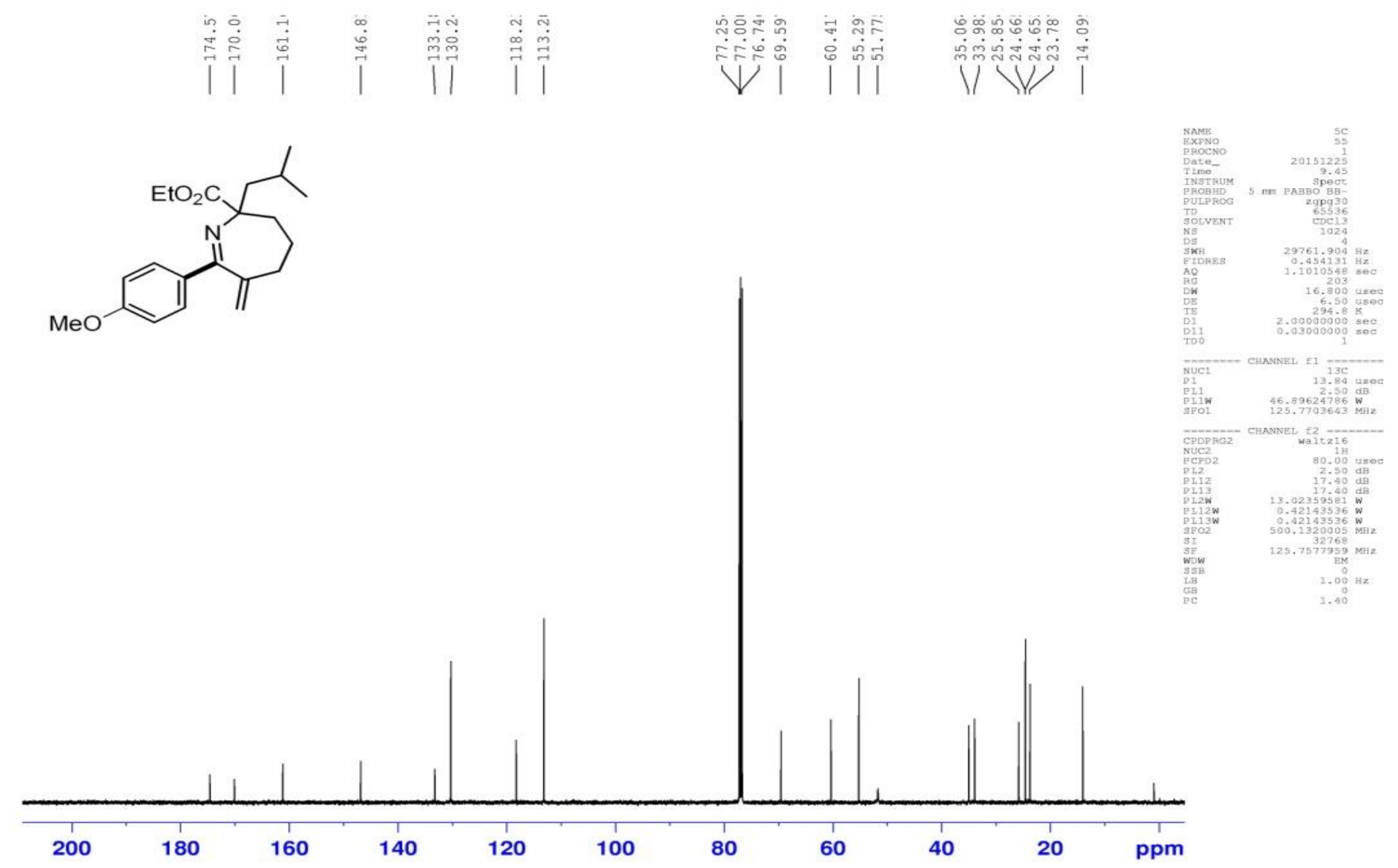




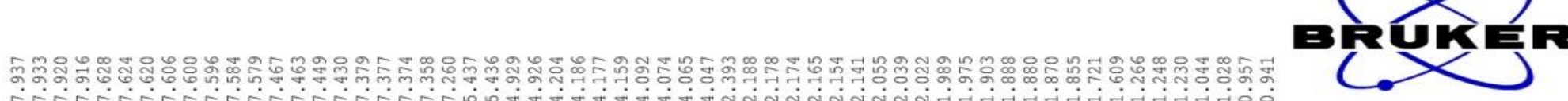

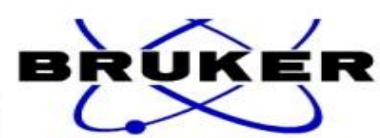<smiles>C=C1CCC(CC(C)C)(CC(C)C)N=C1c1ccc(-c2ccccc2)cc1</smiles>

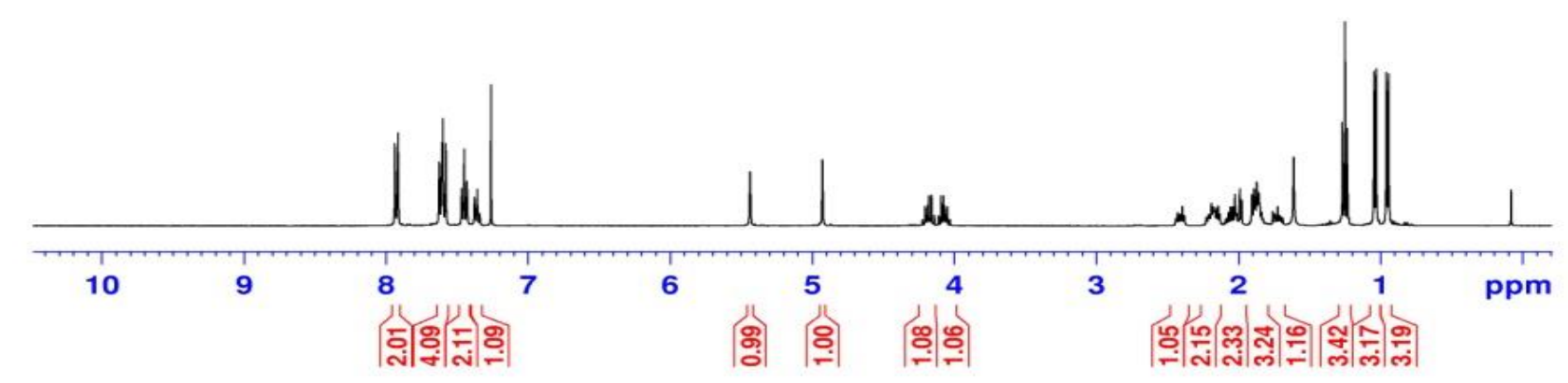




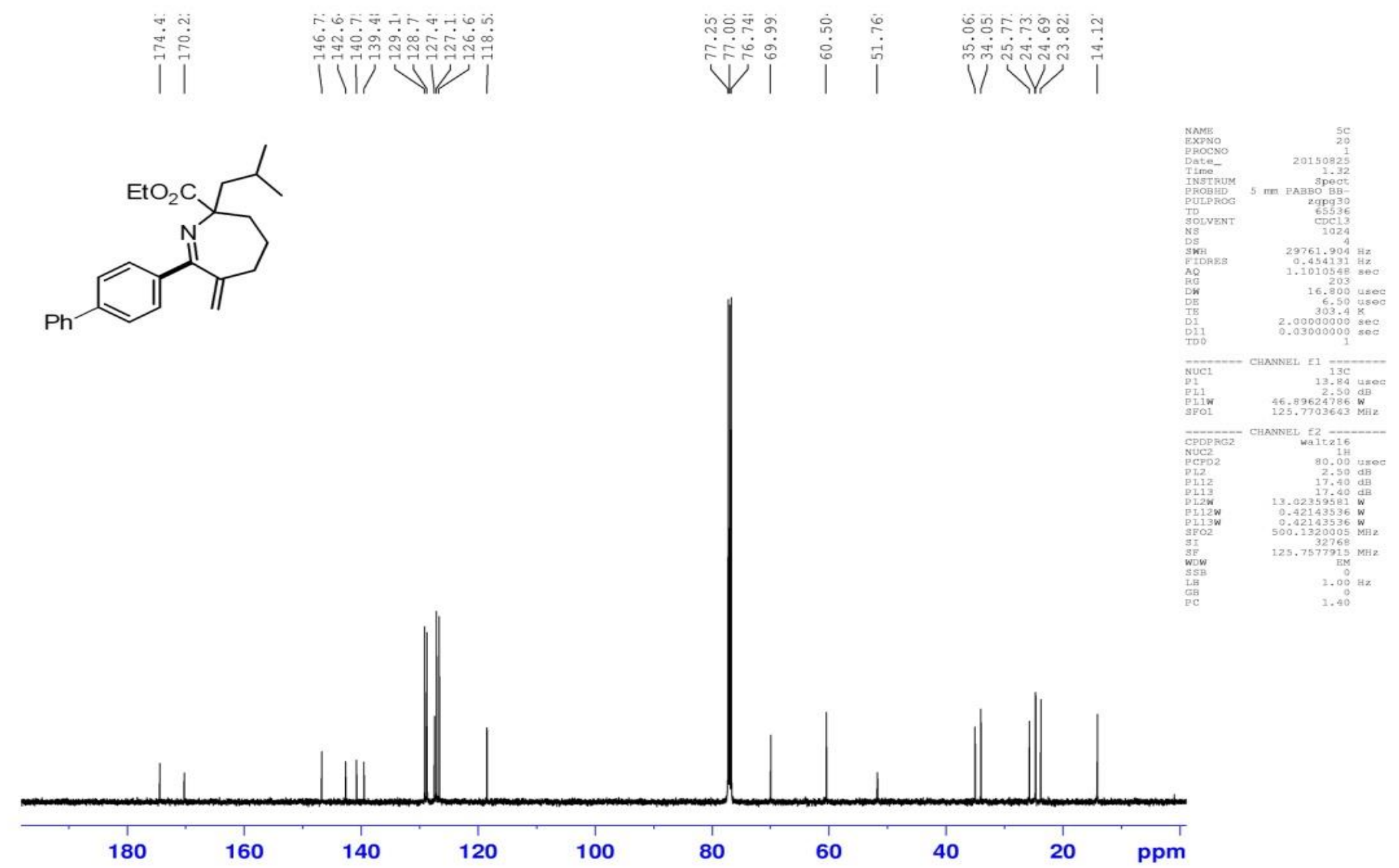




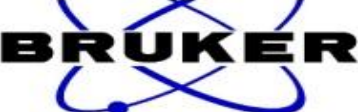

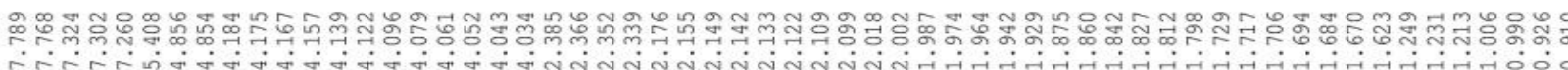
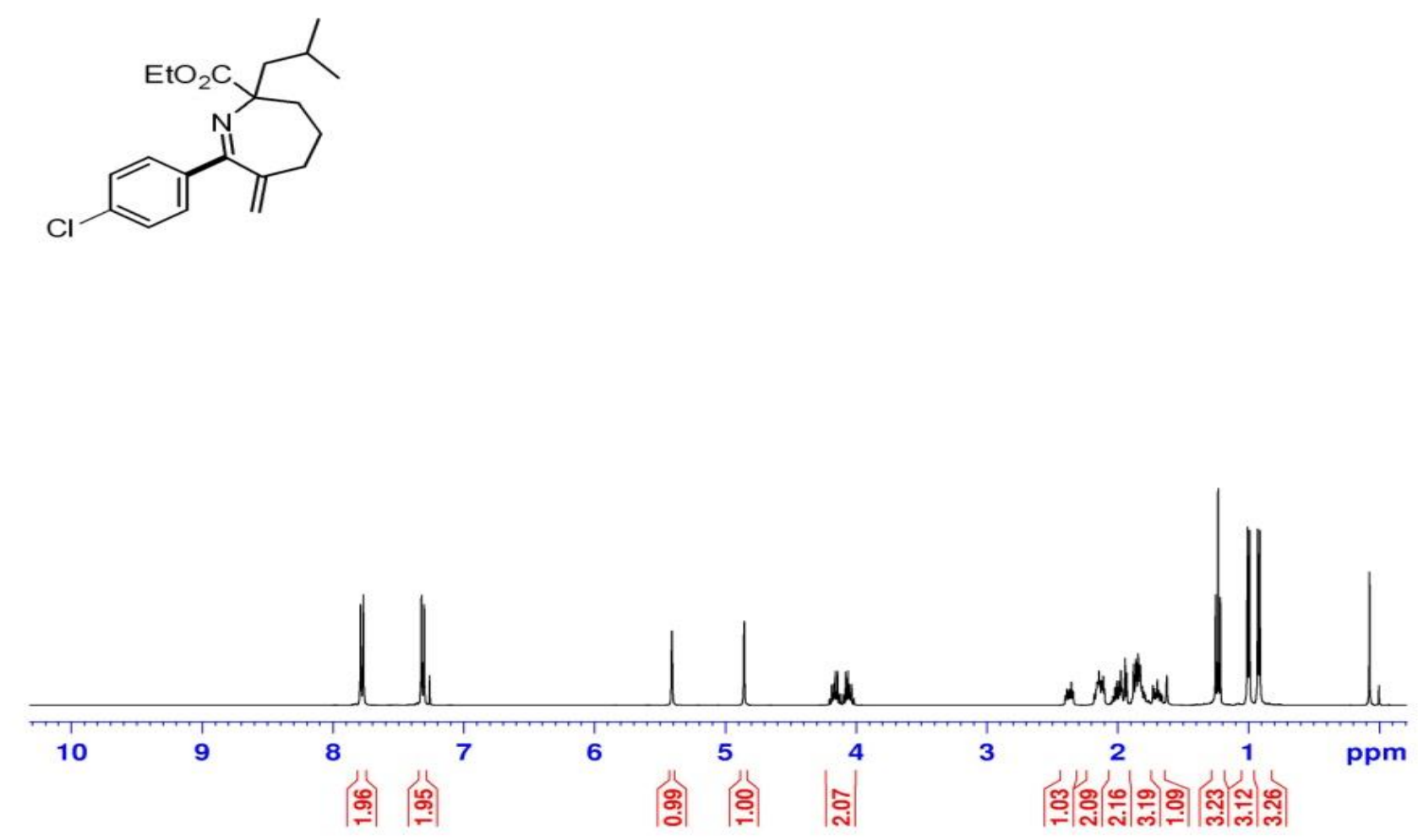


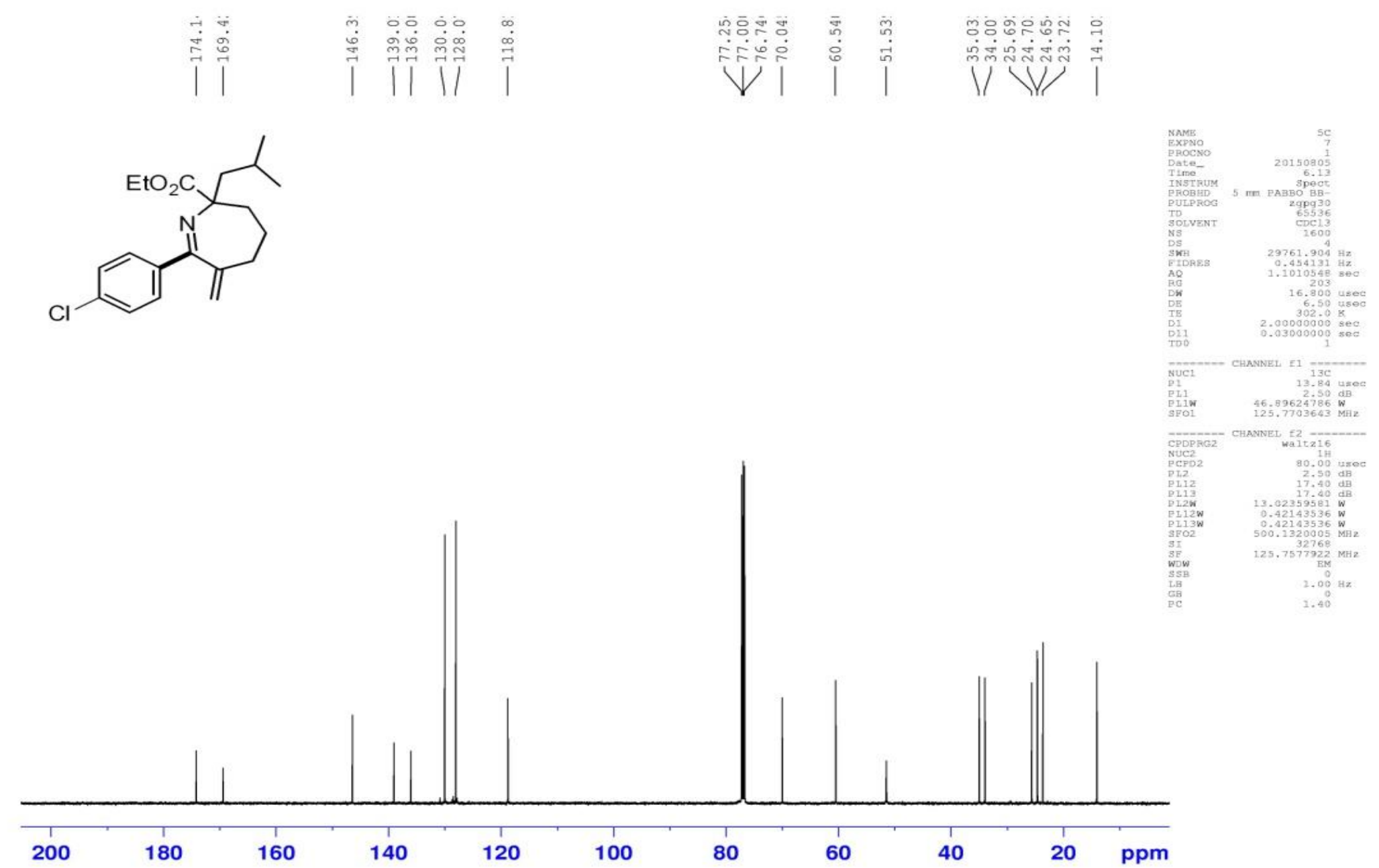



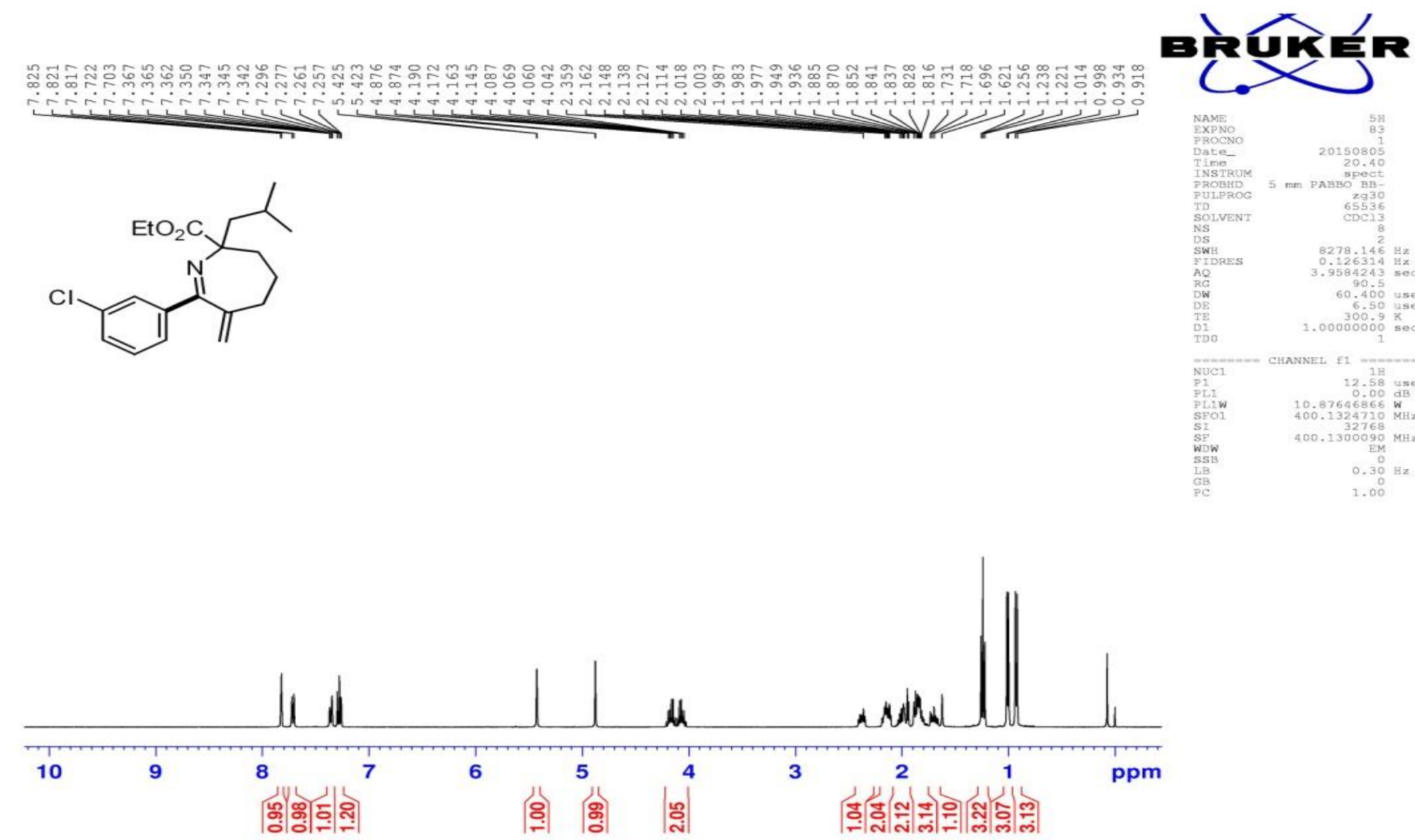


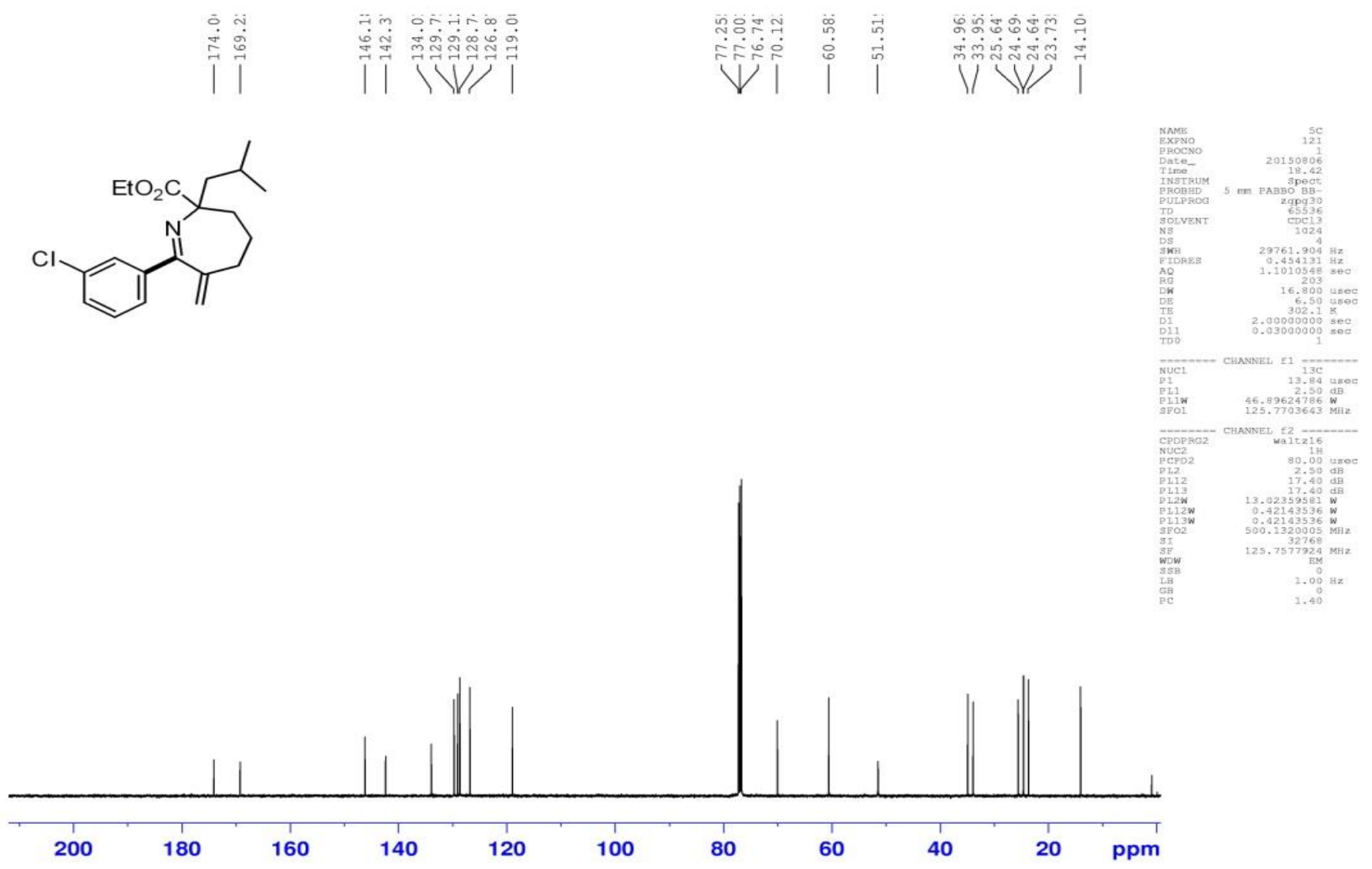




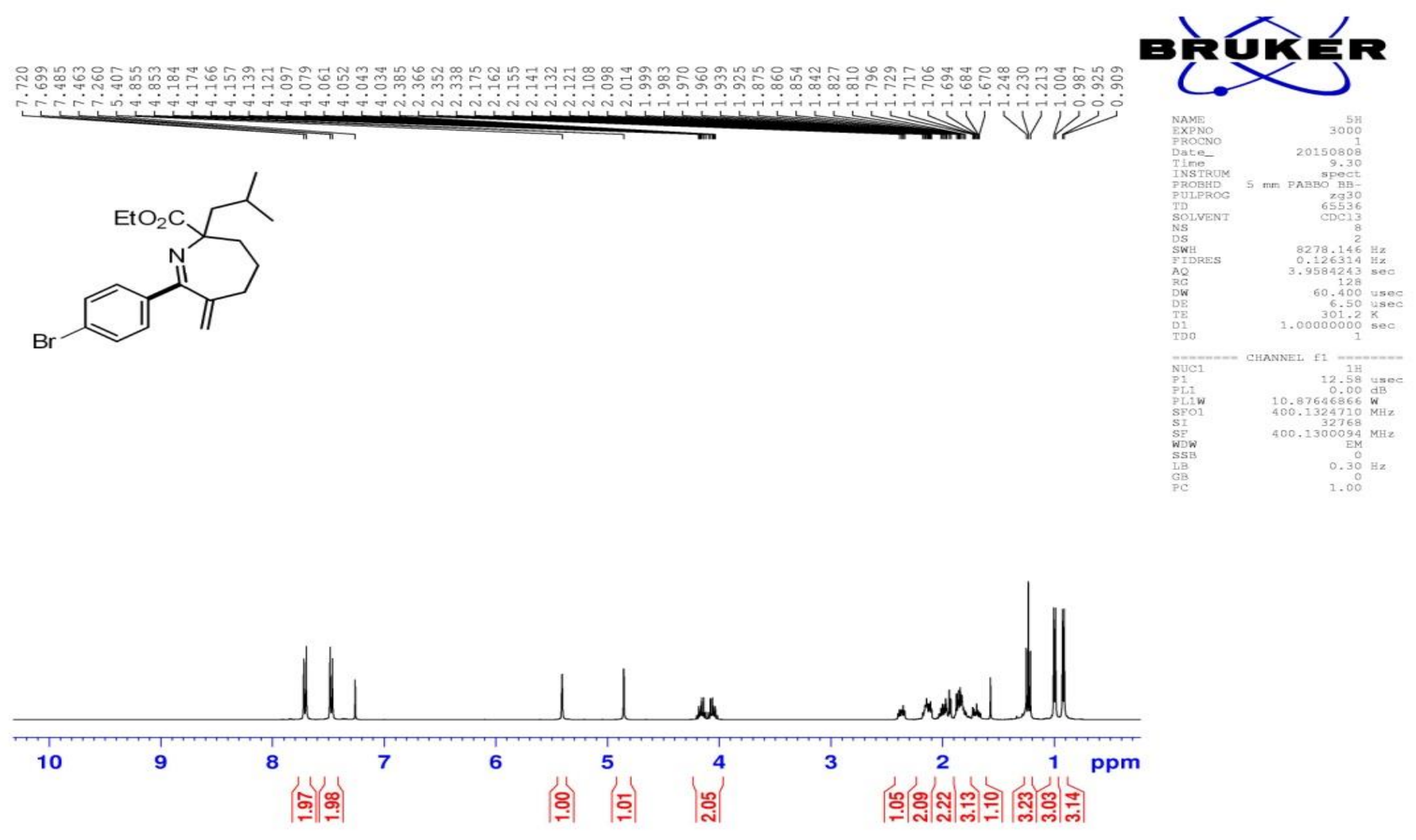




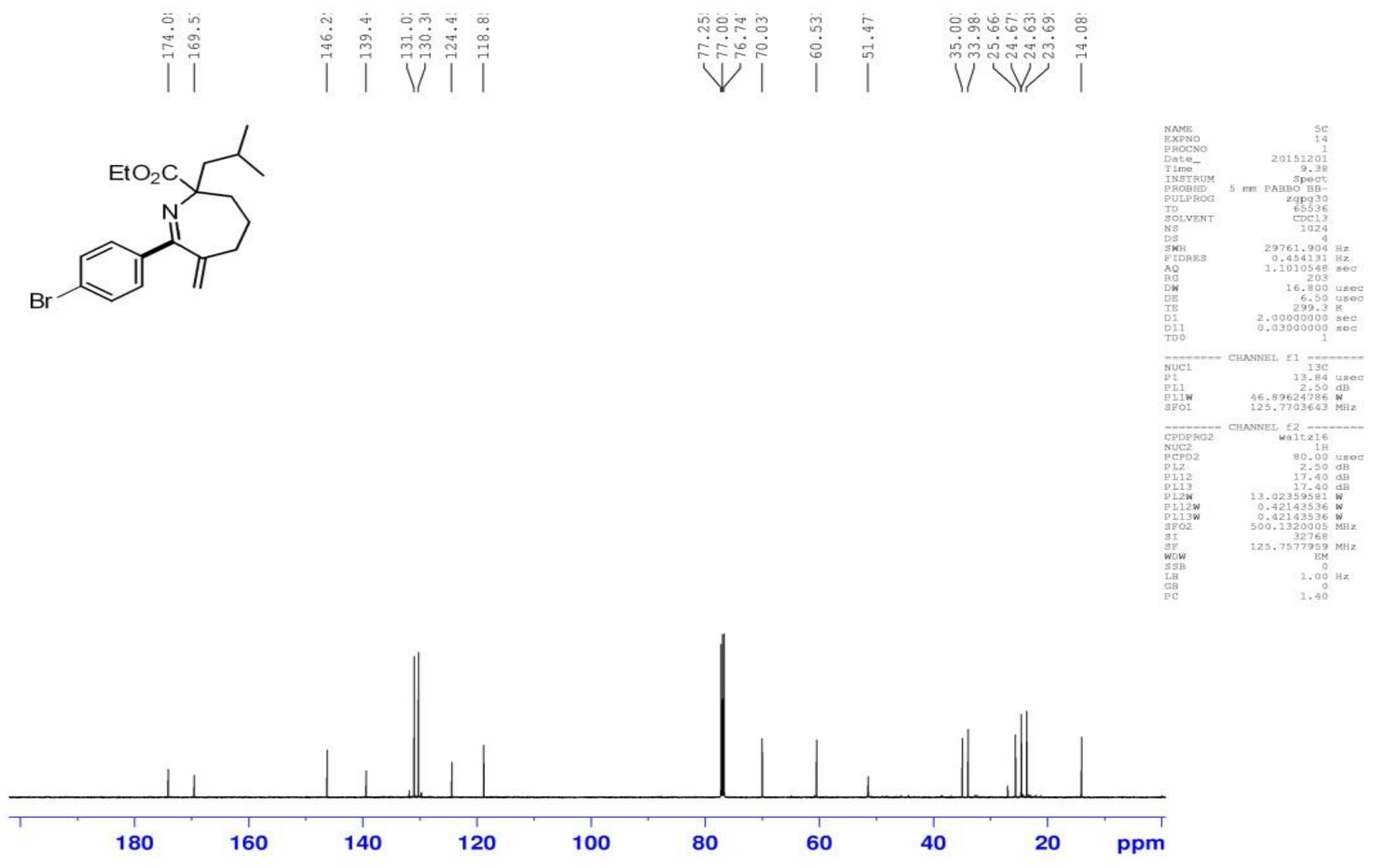




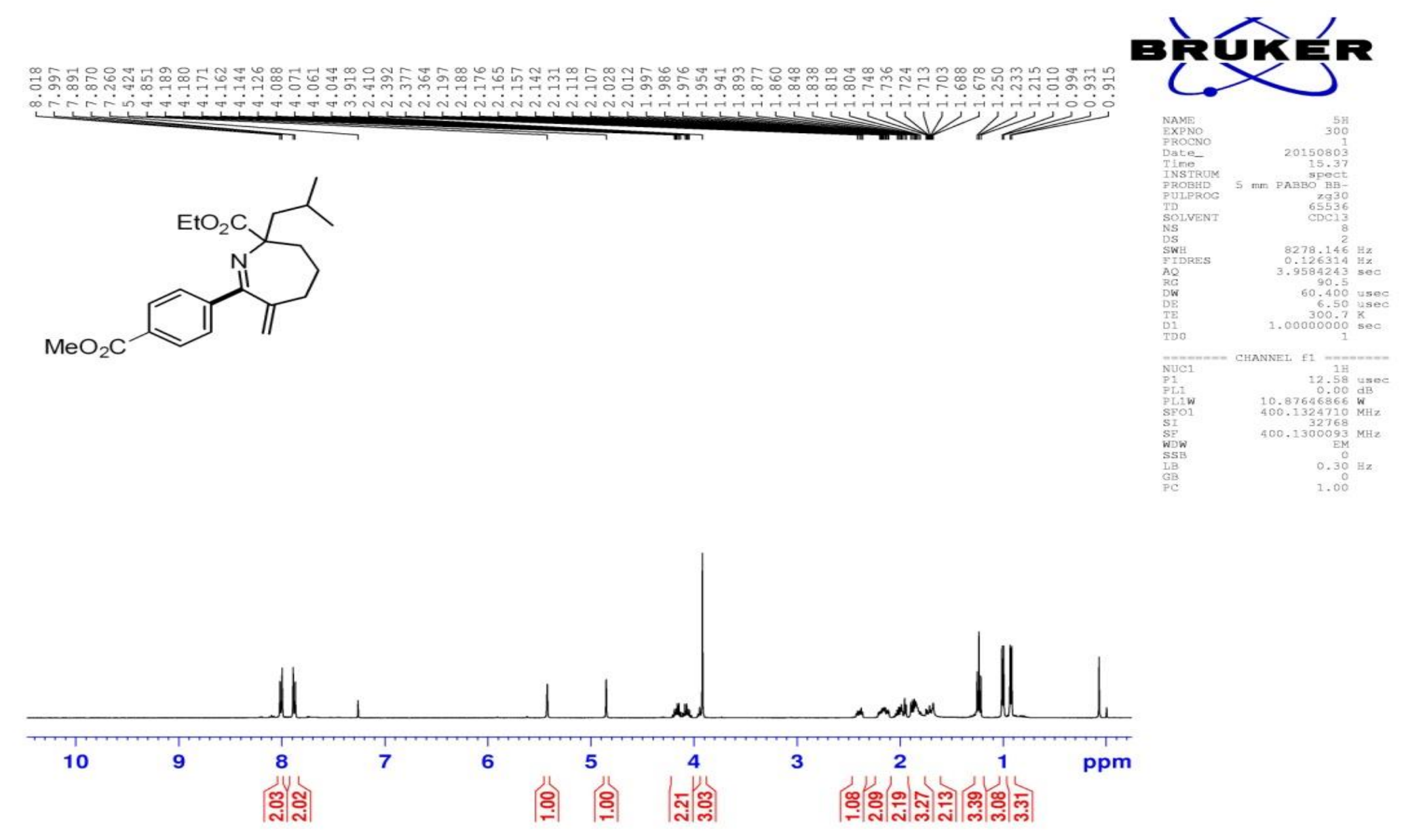




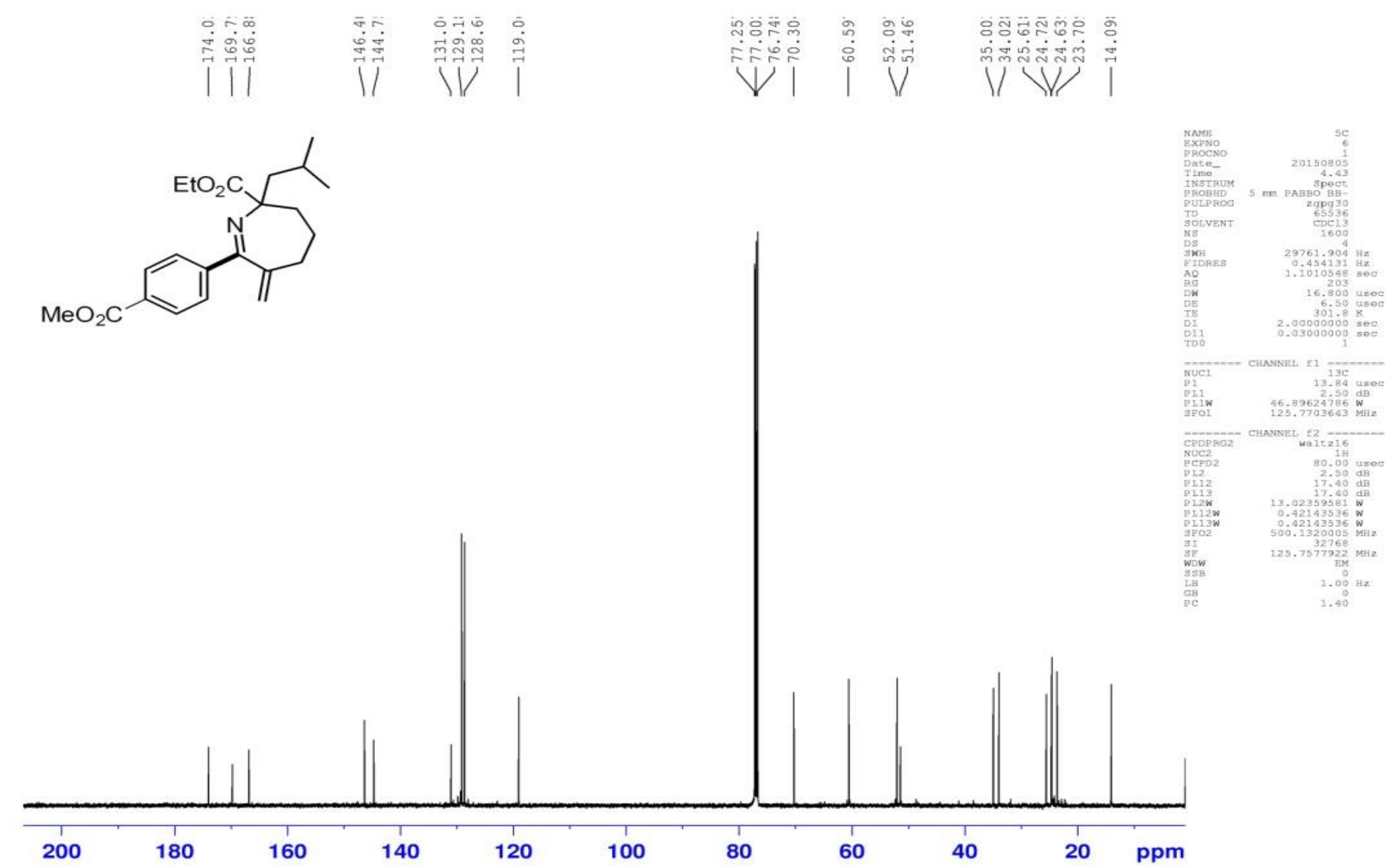




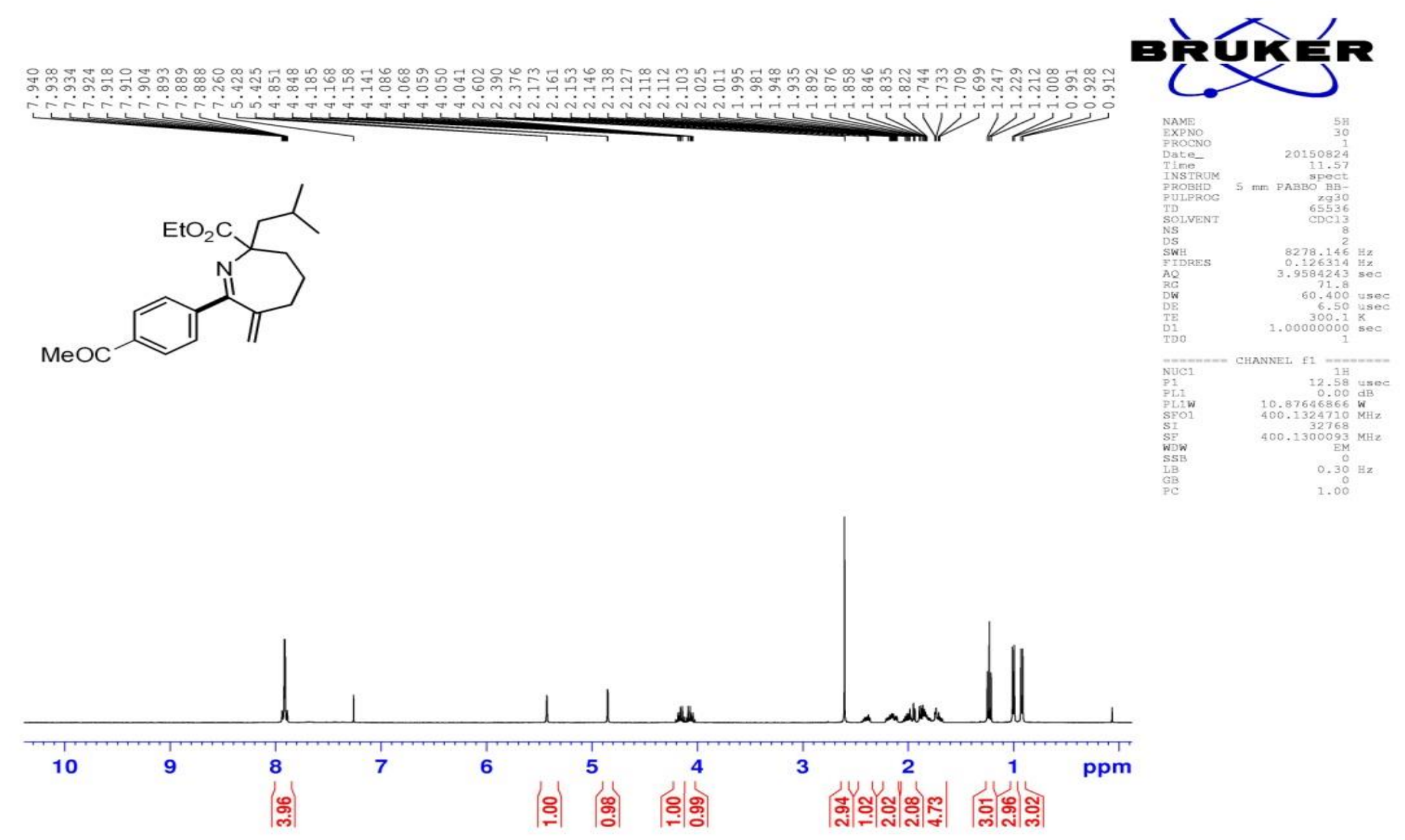




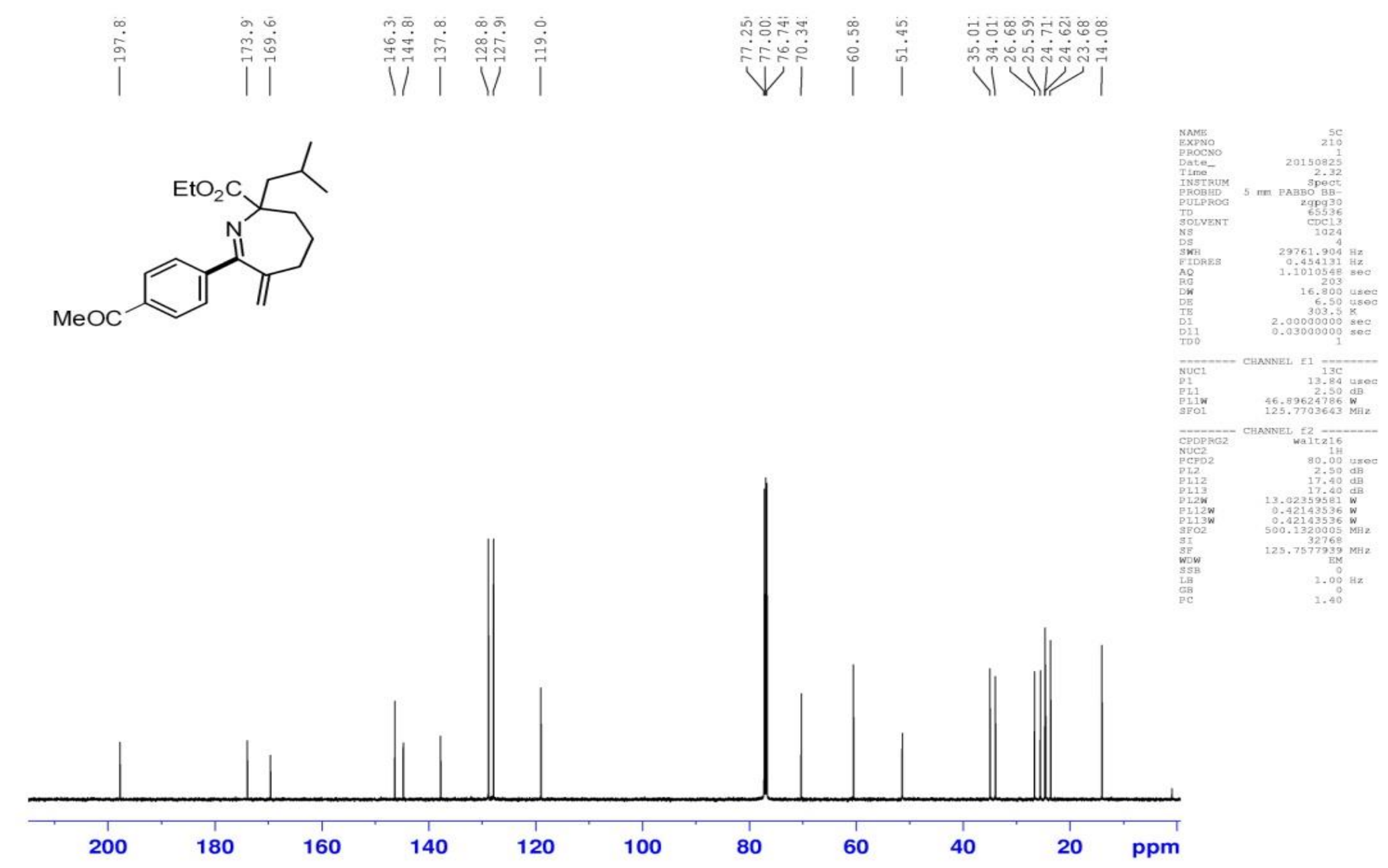




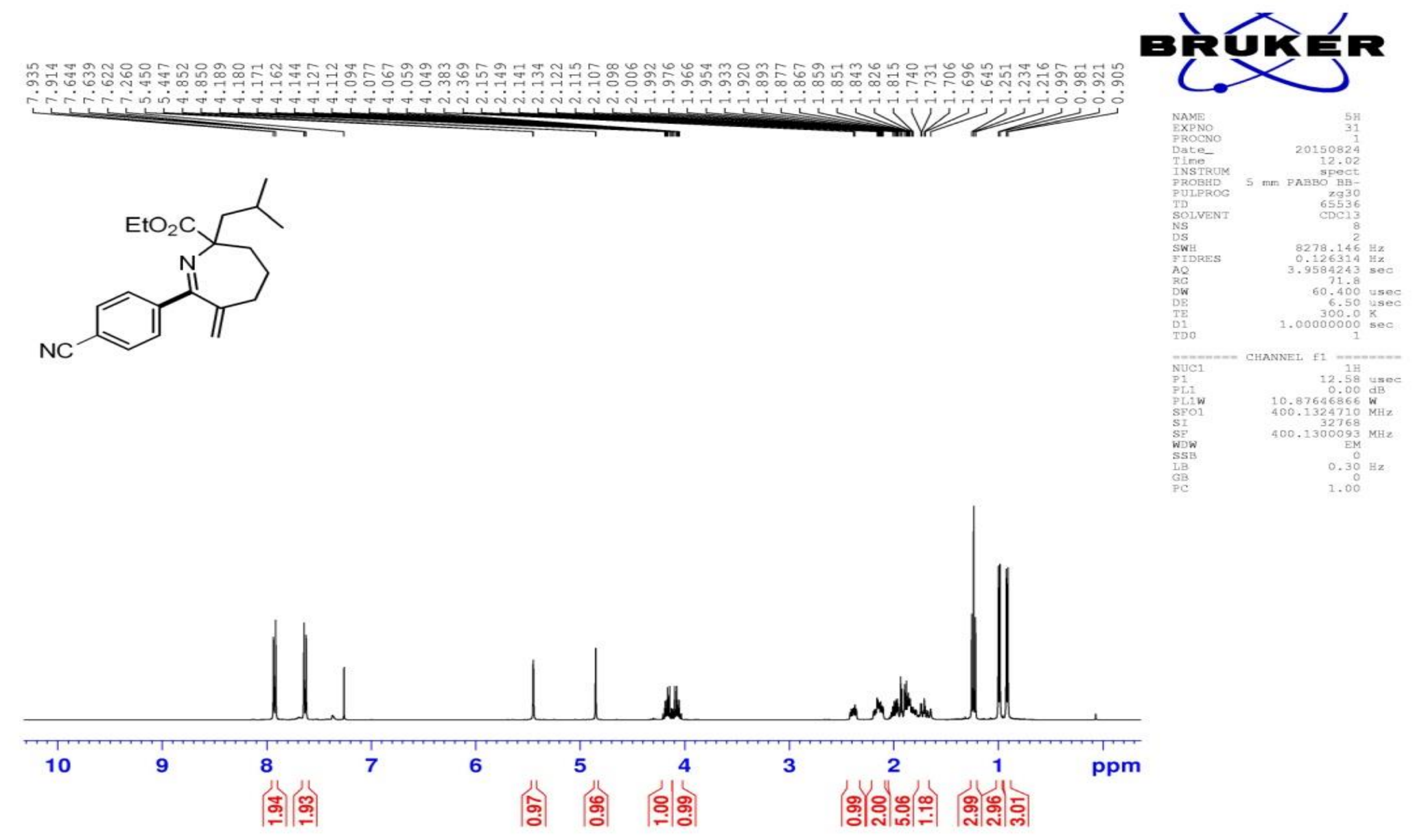




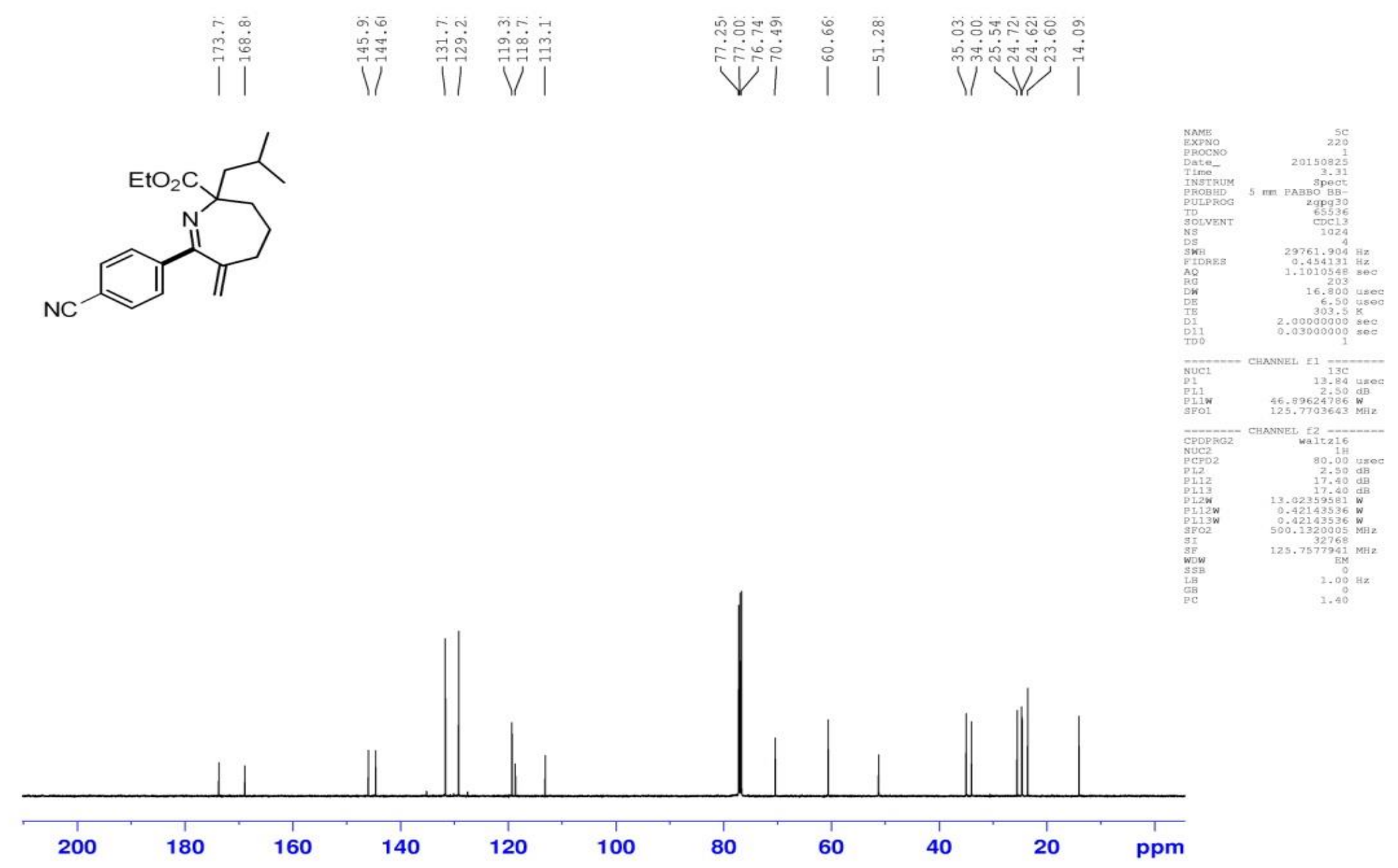




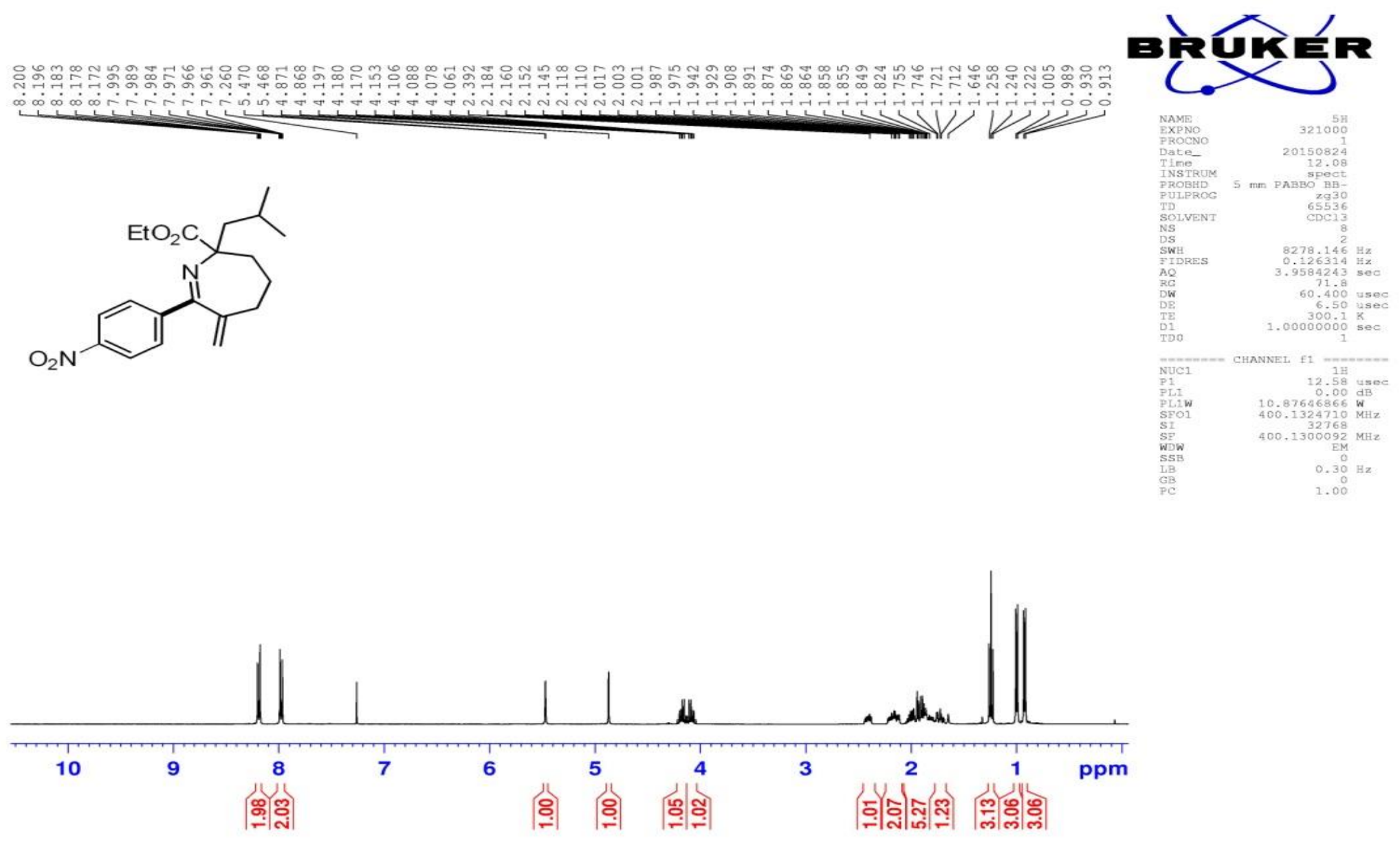




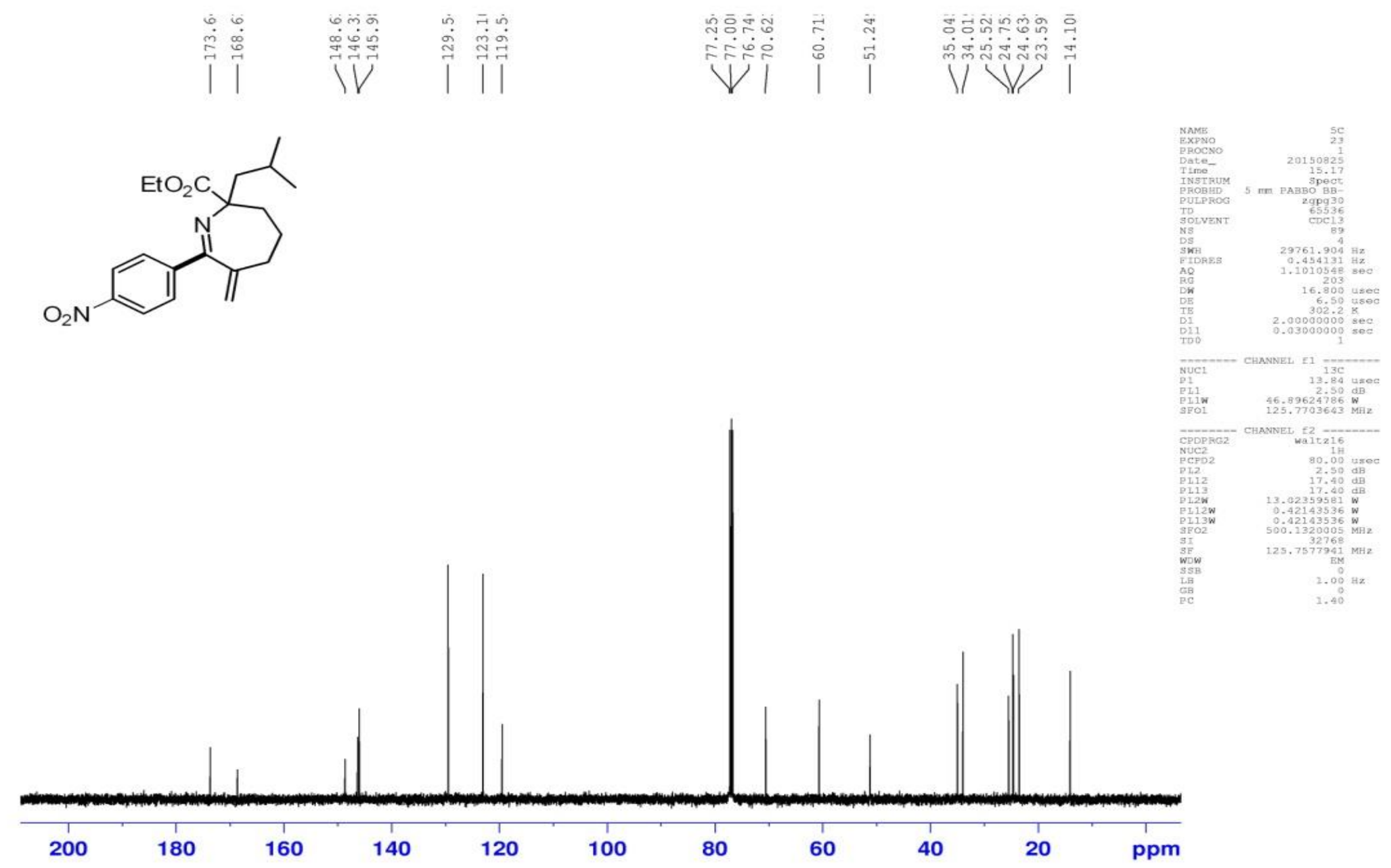




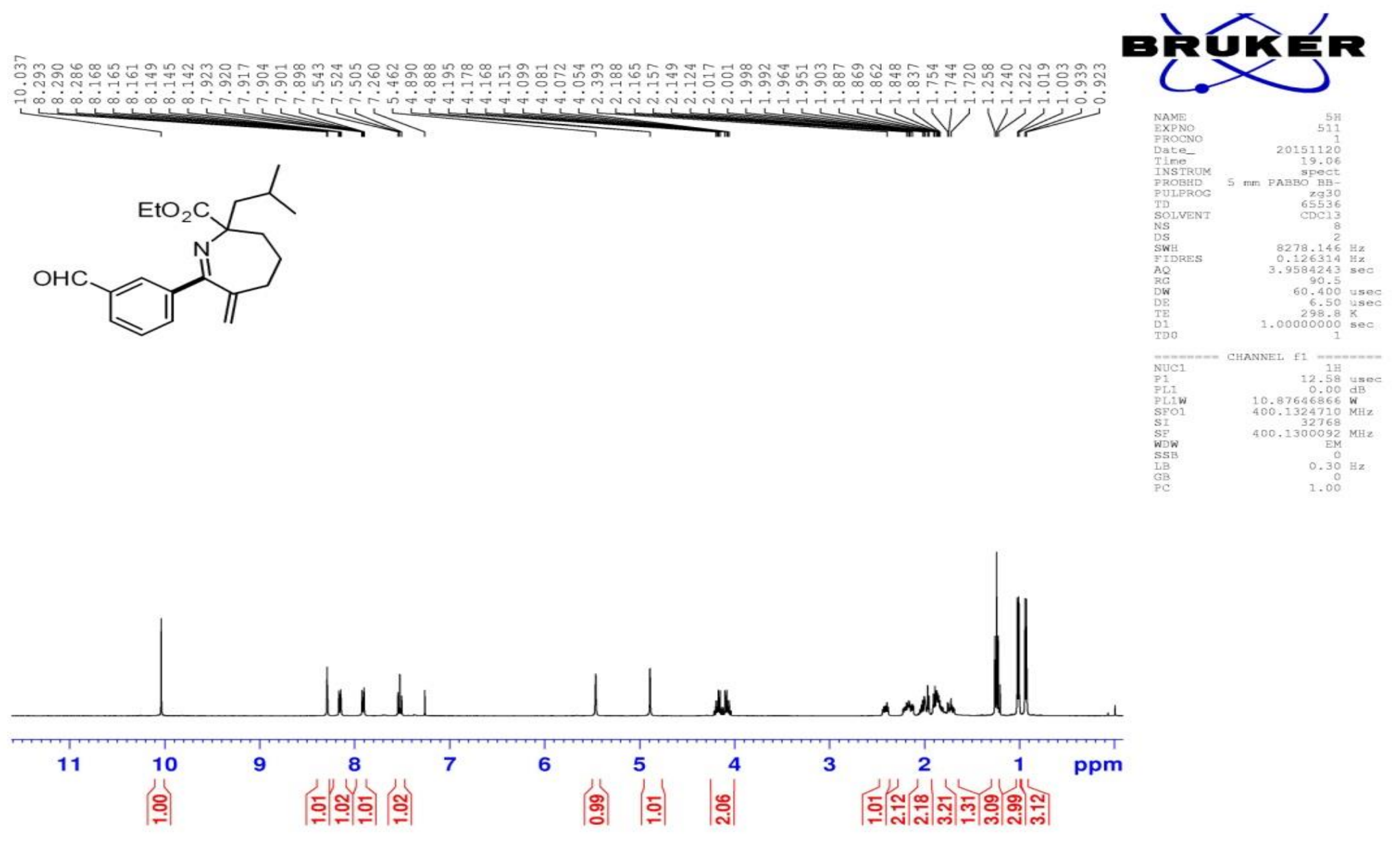




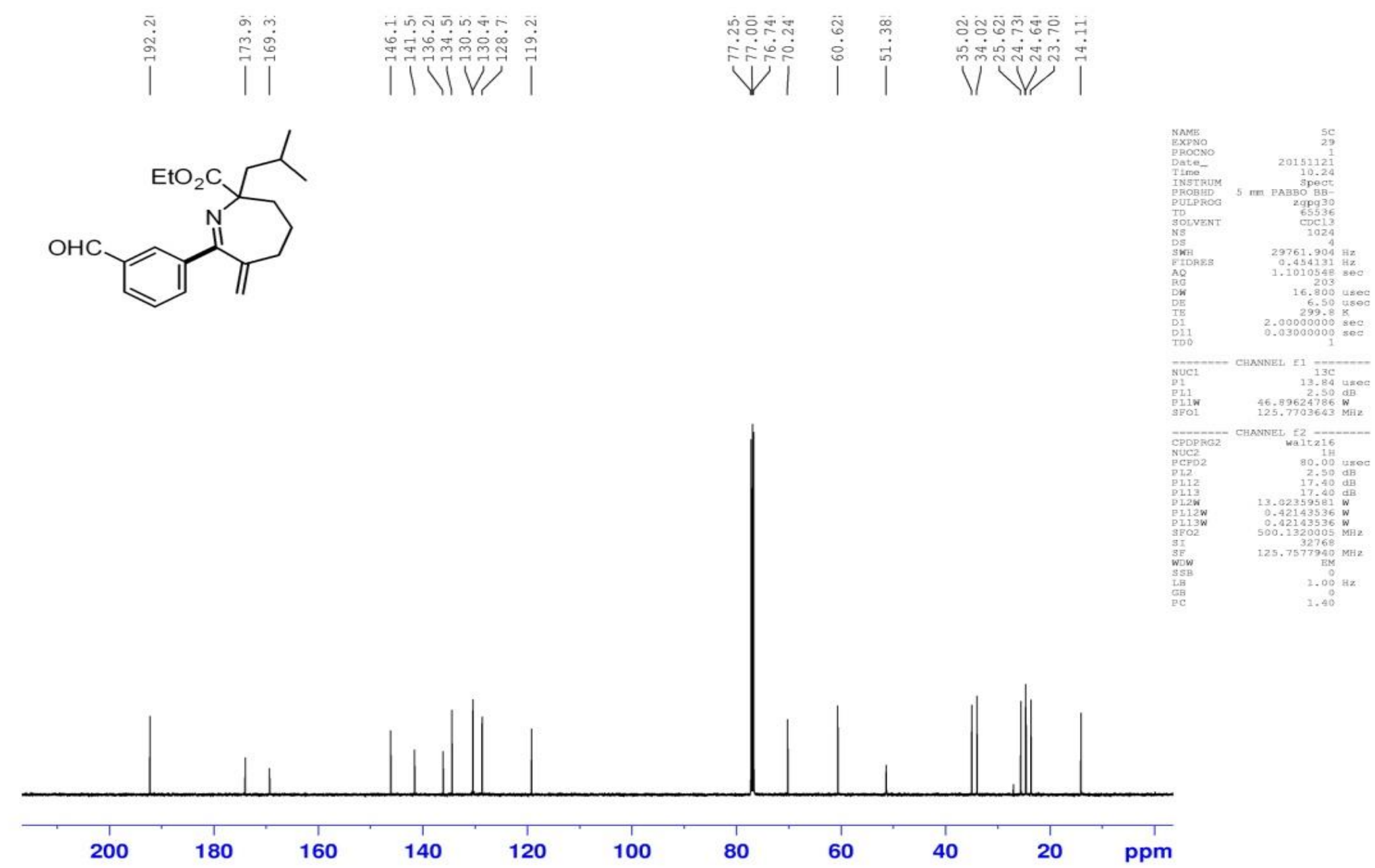




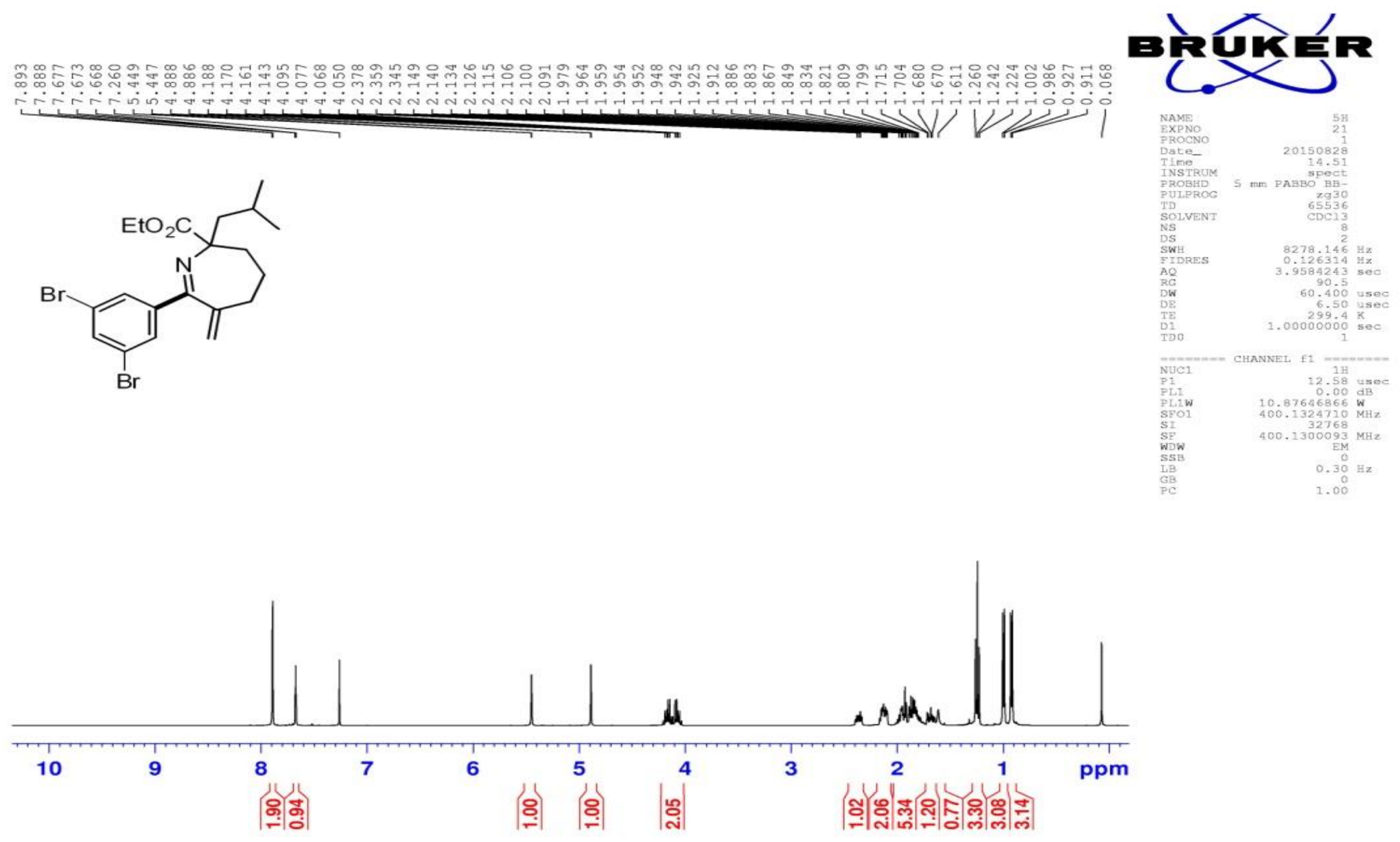




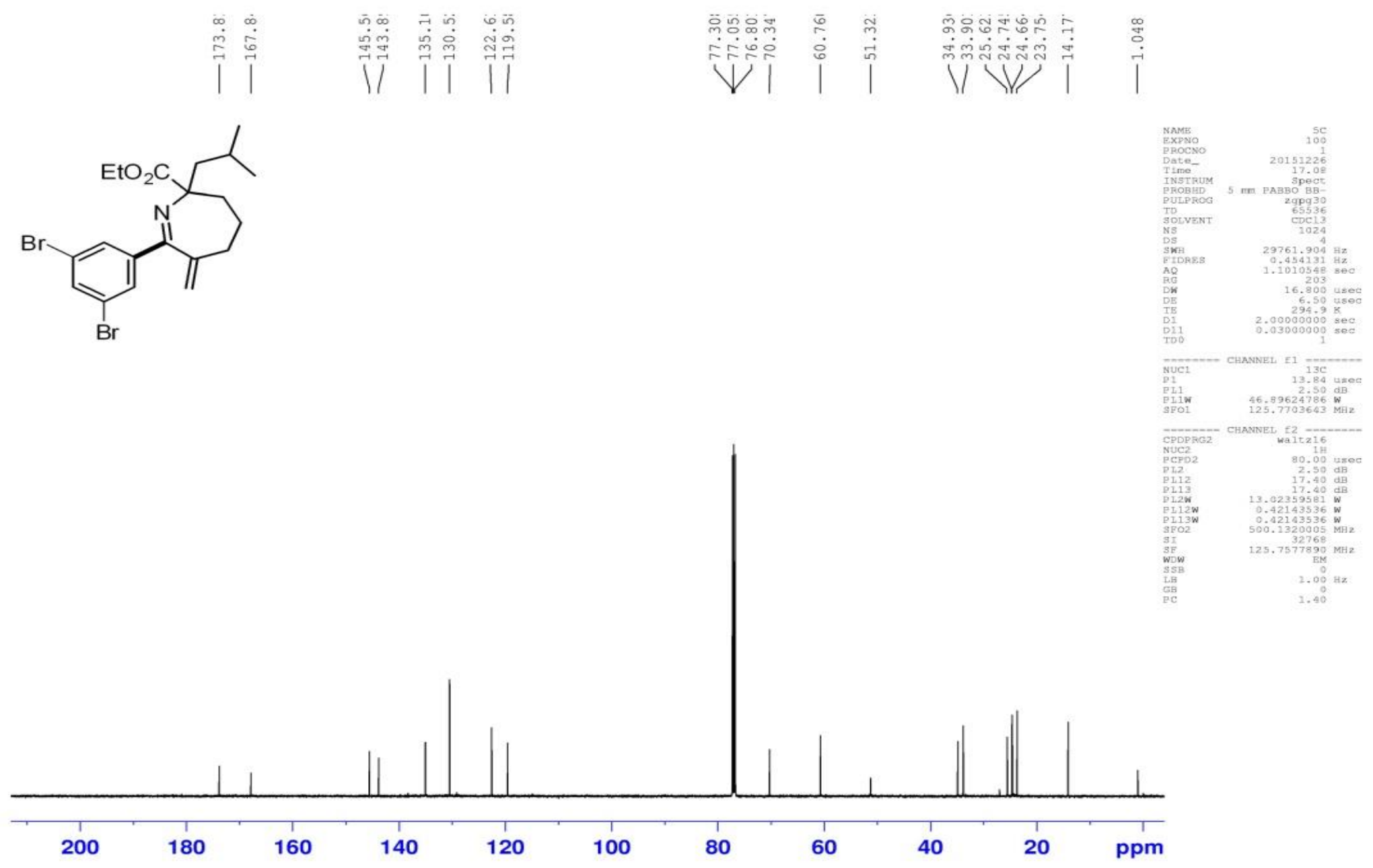




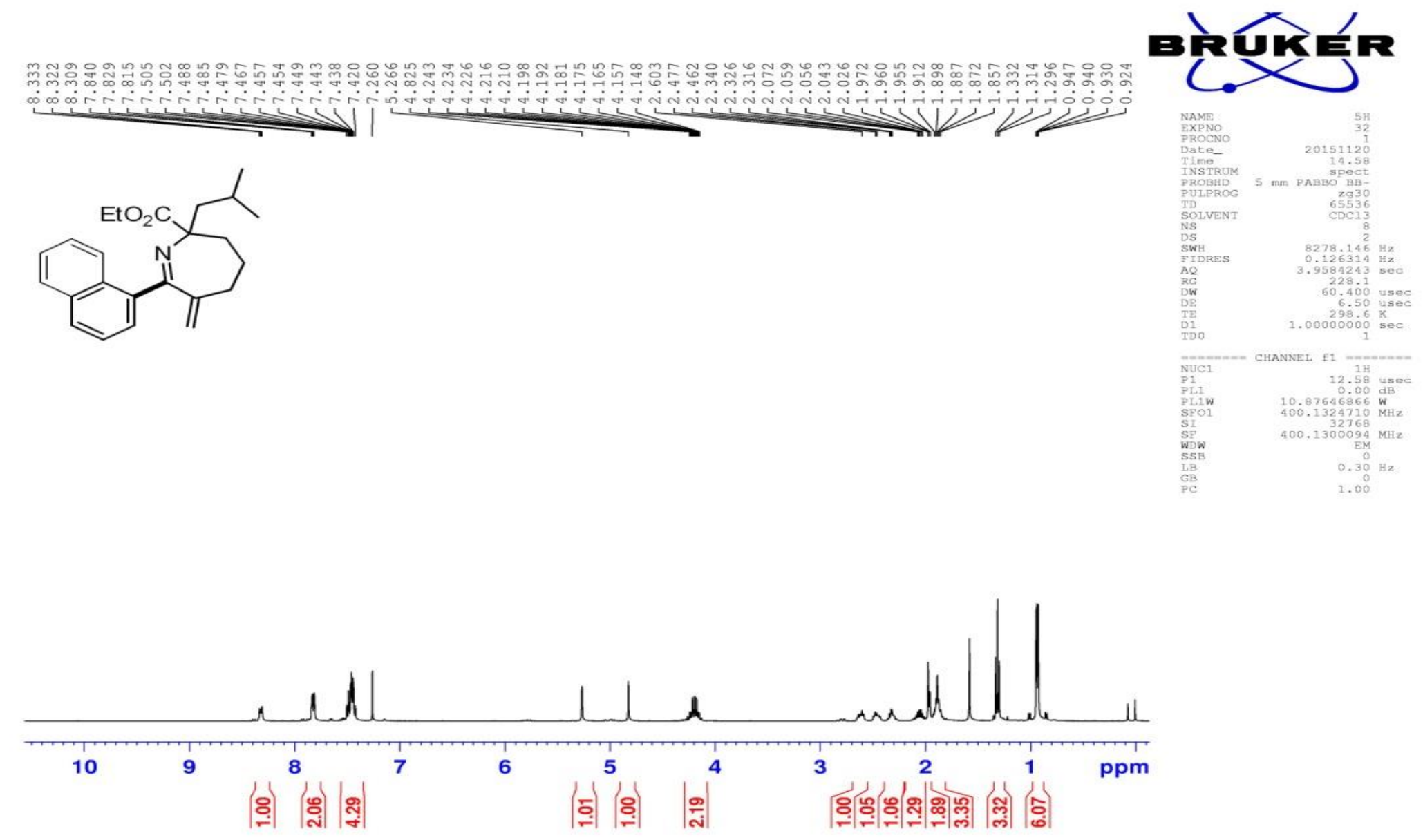




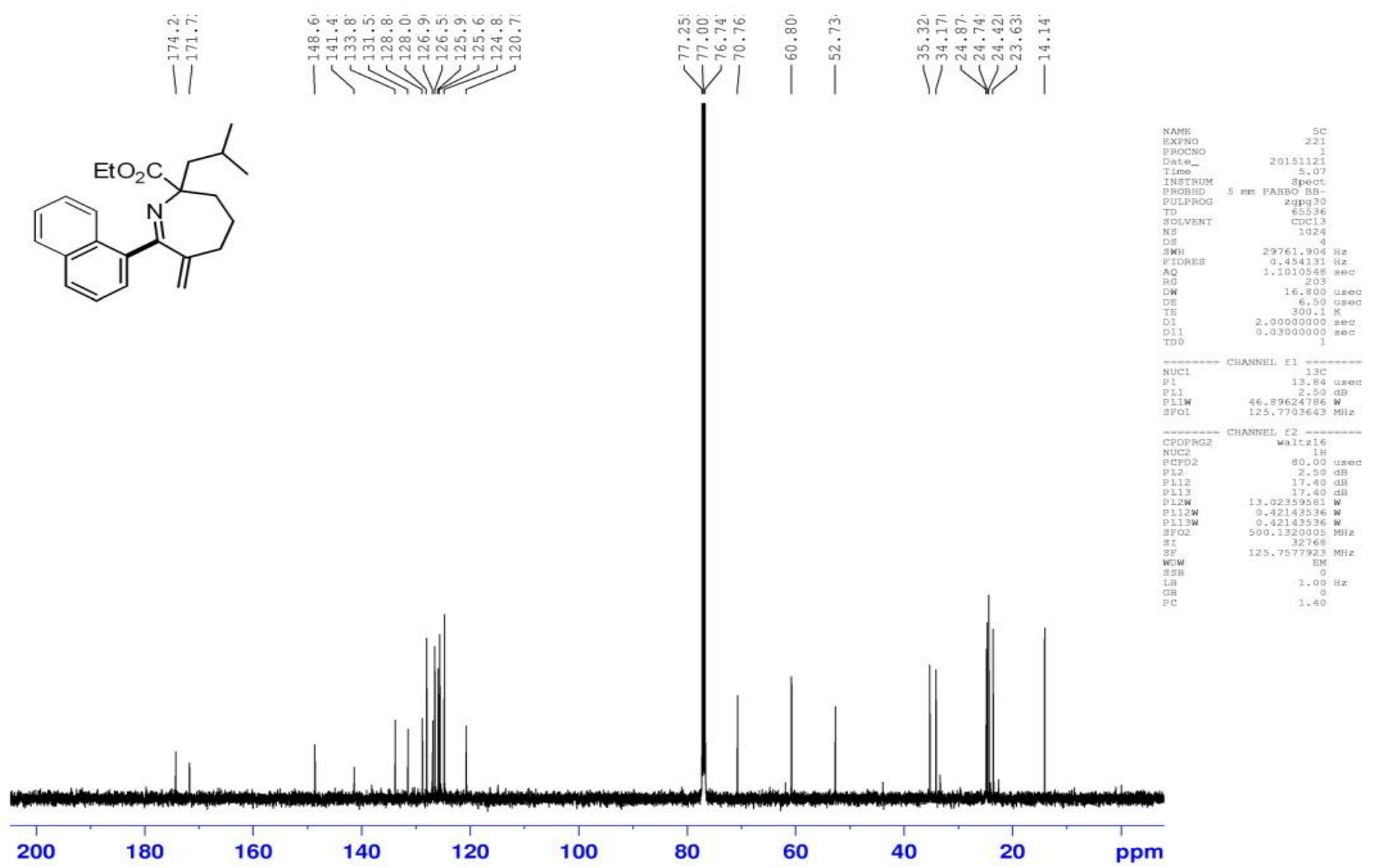




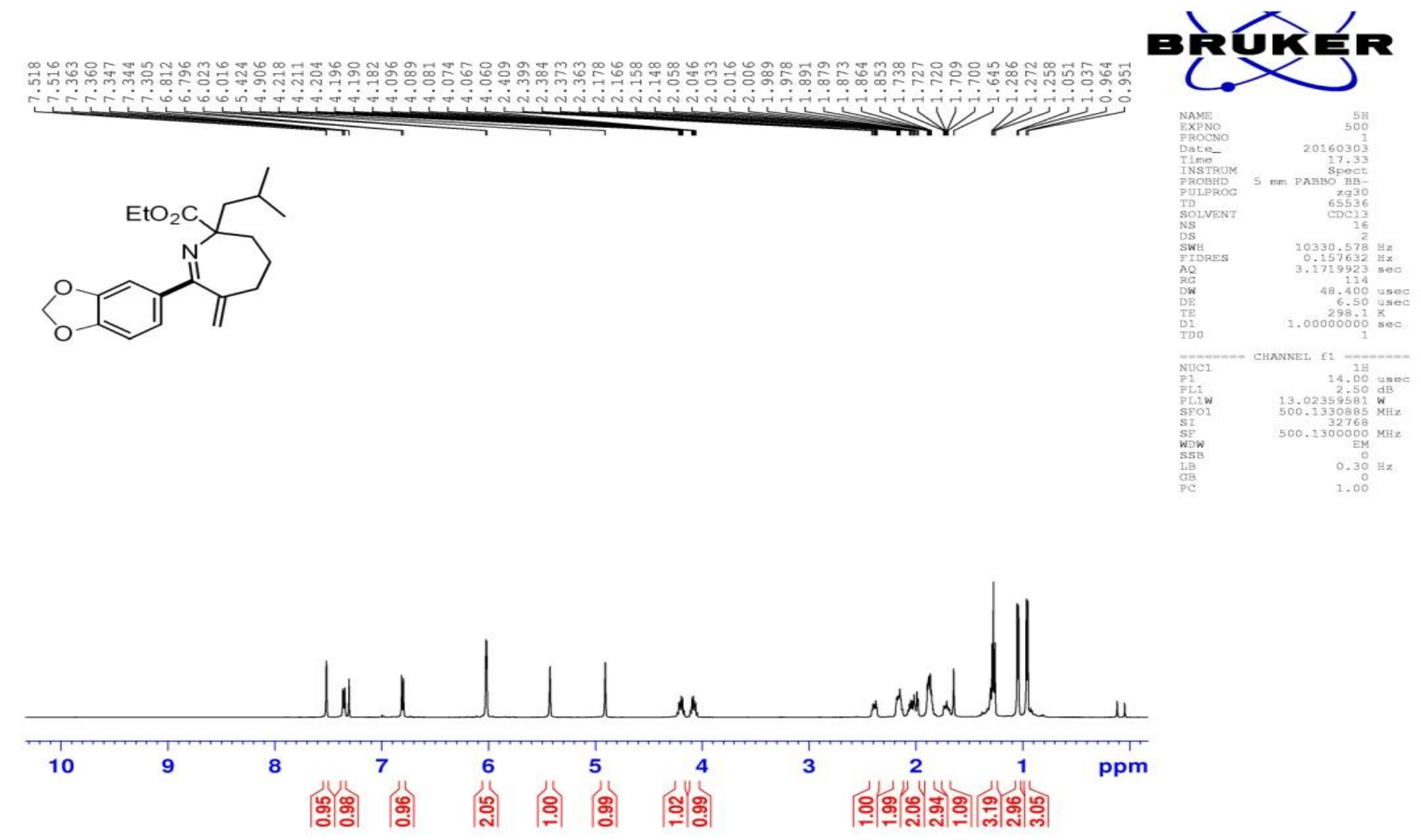




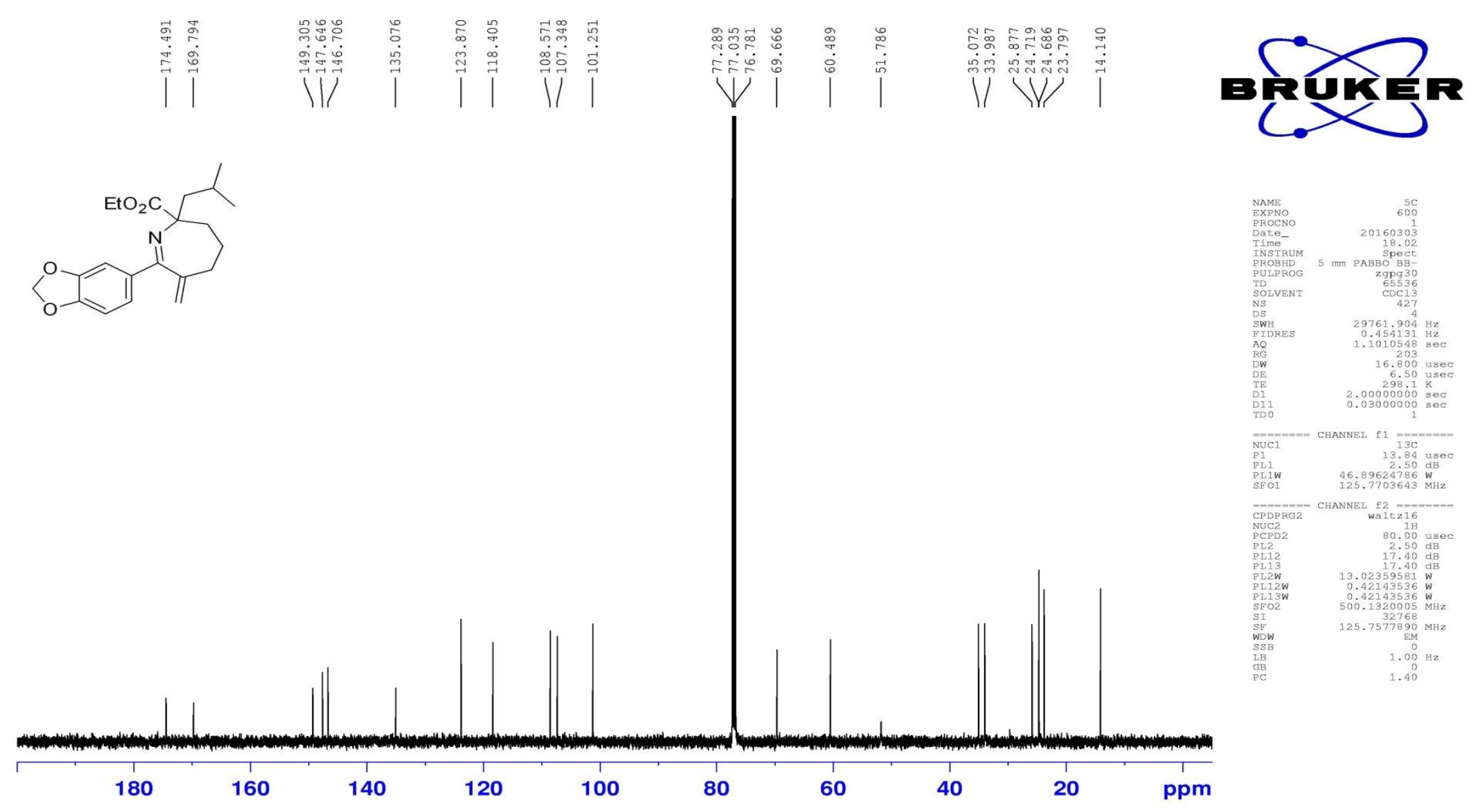




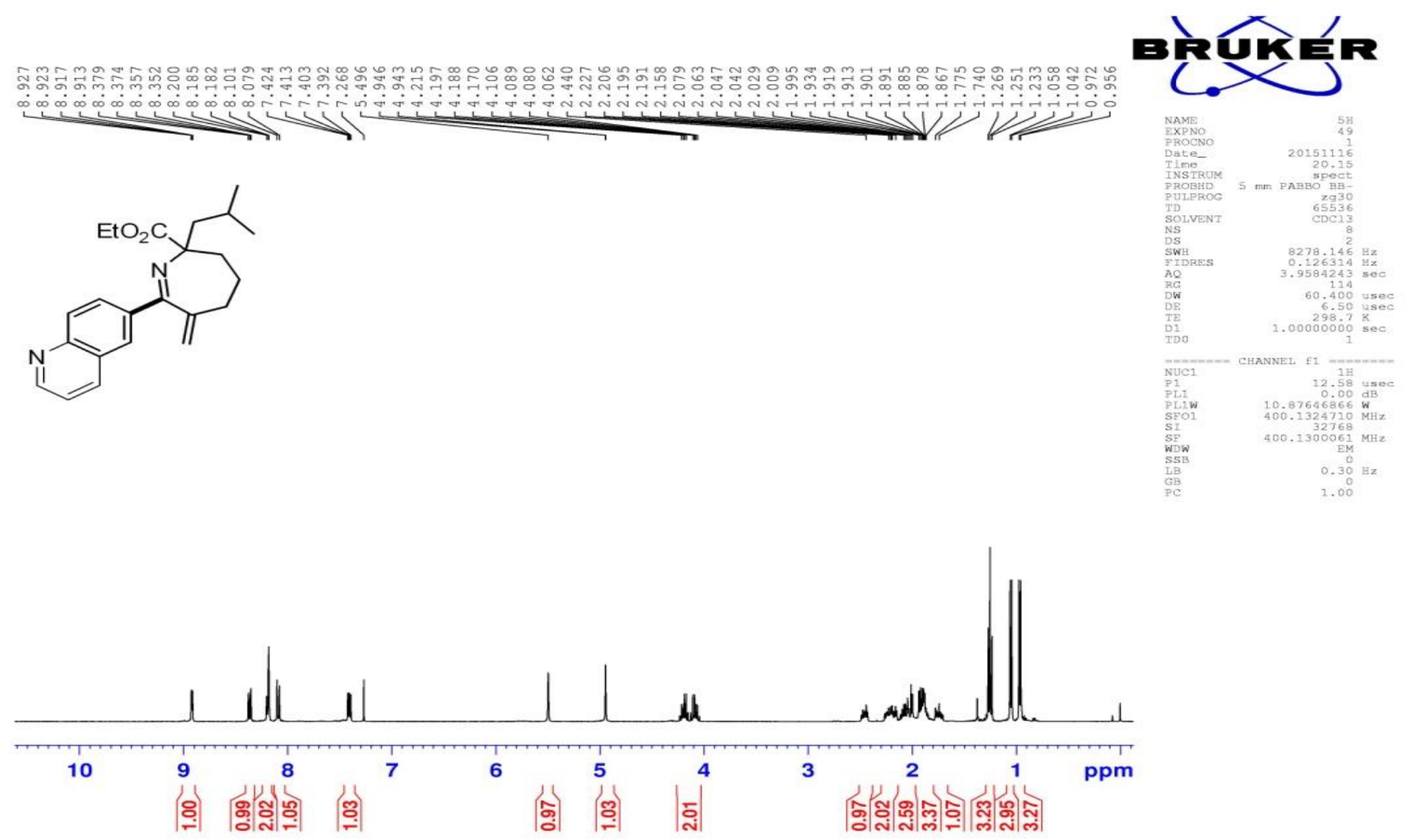




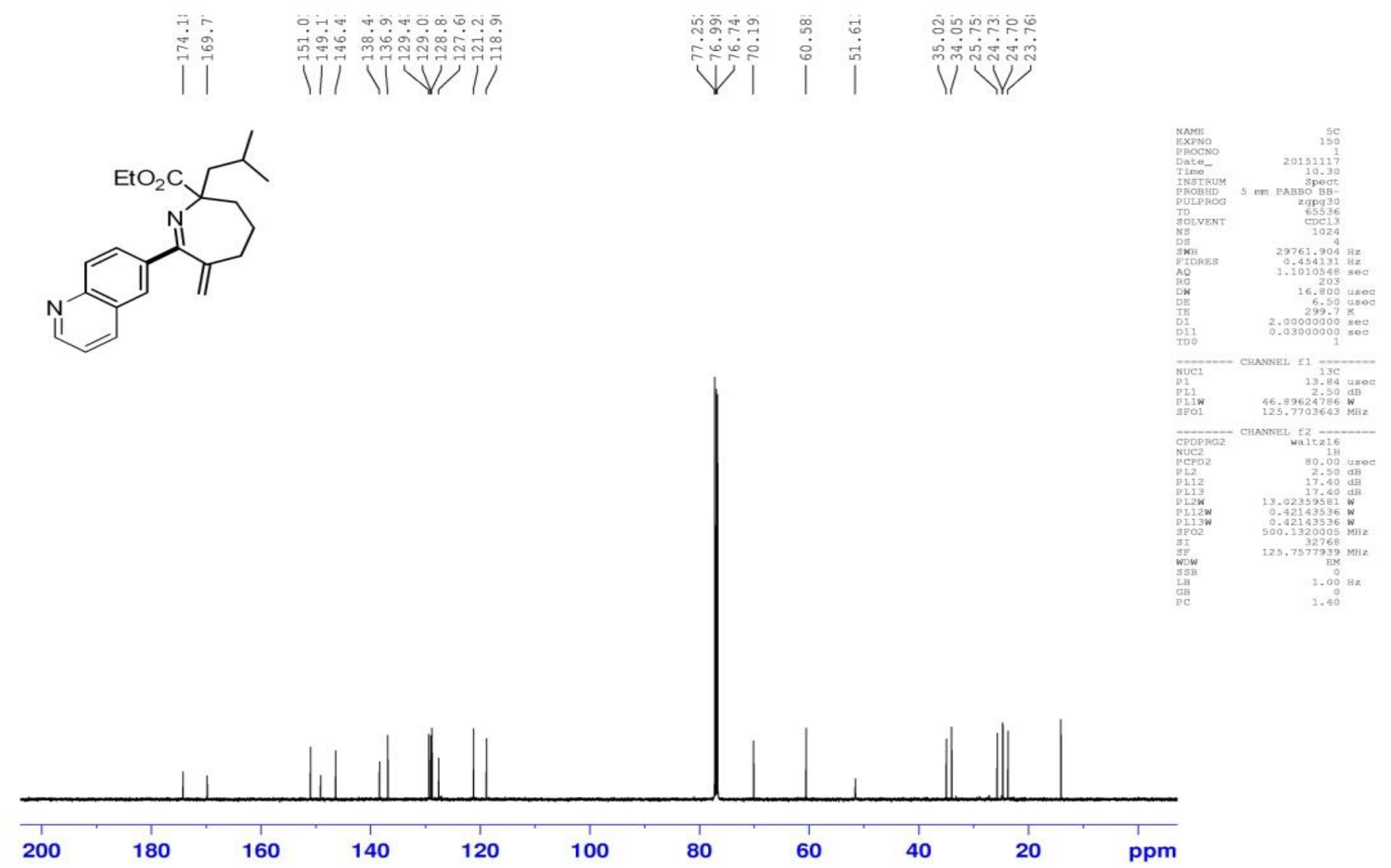




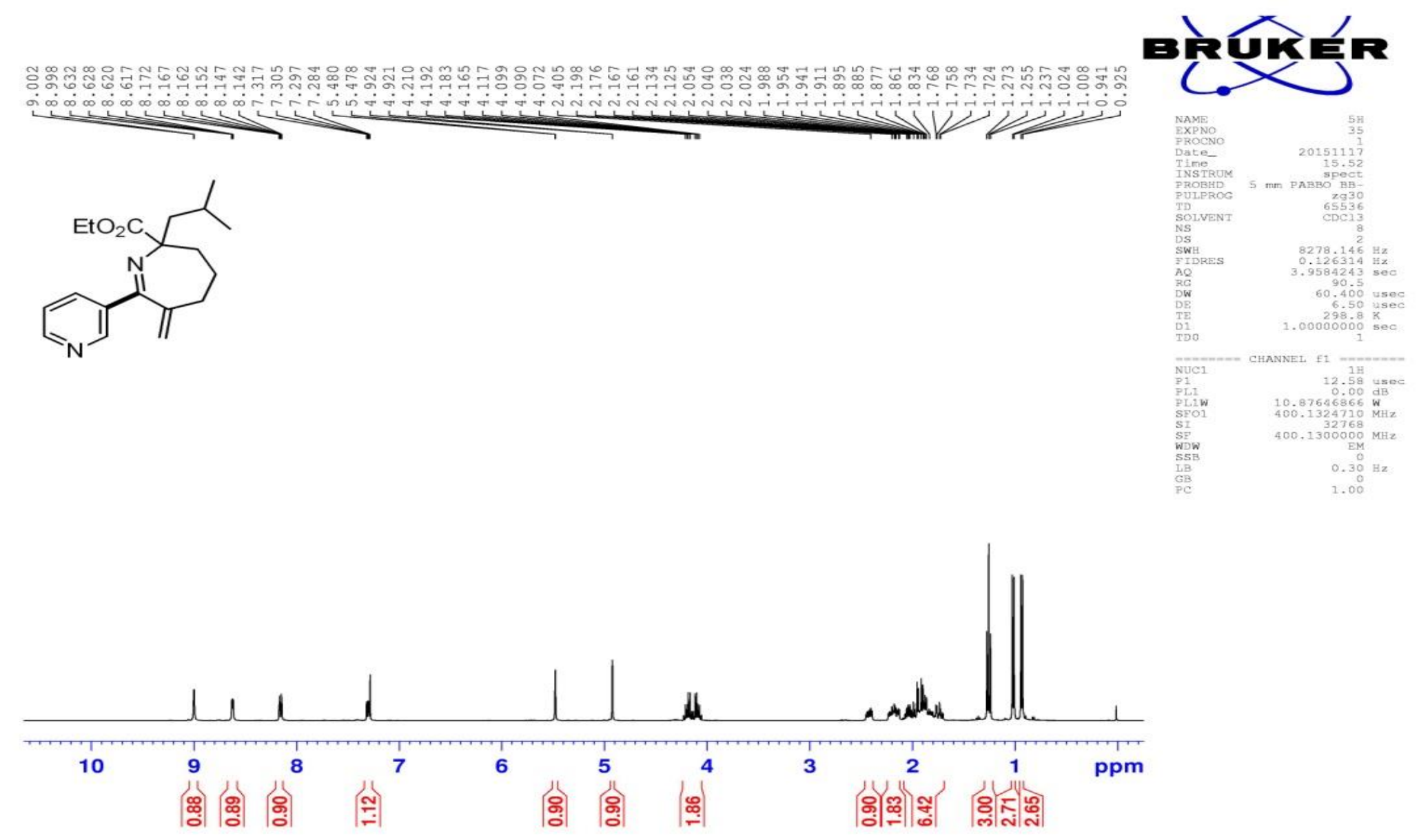




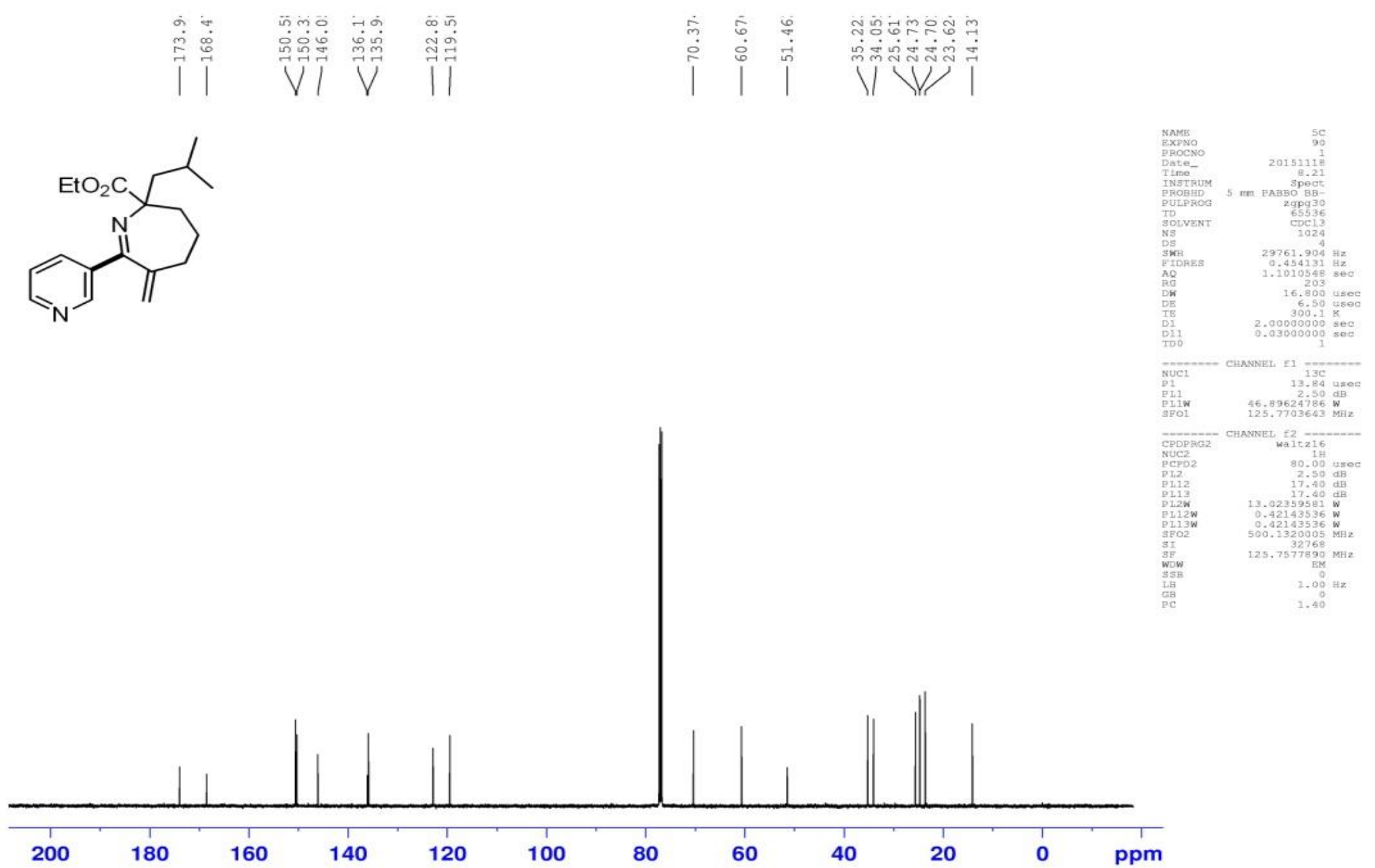




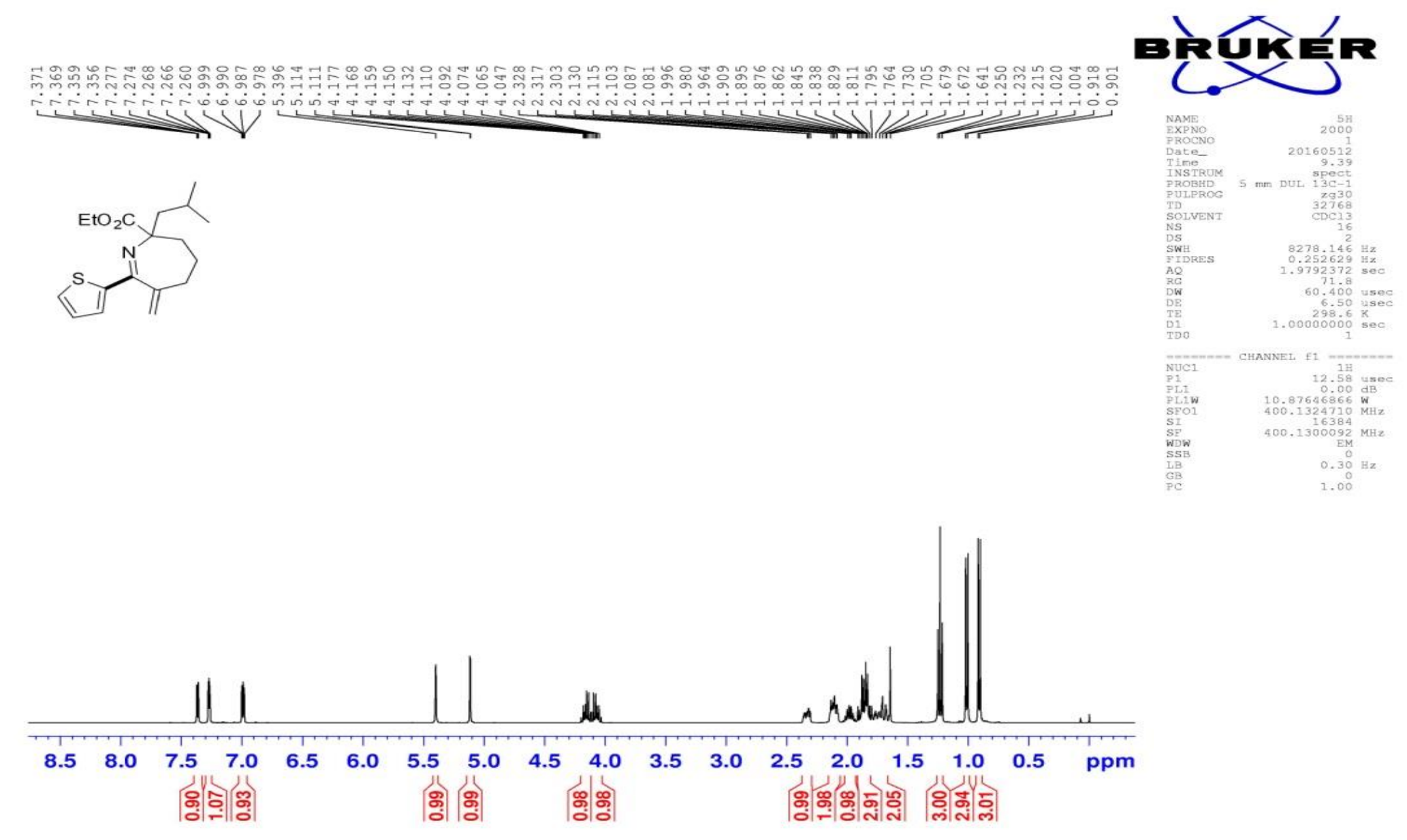




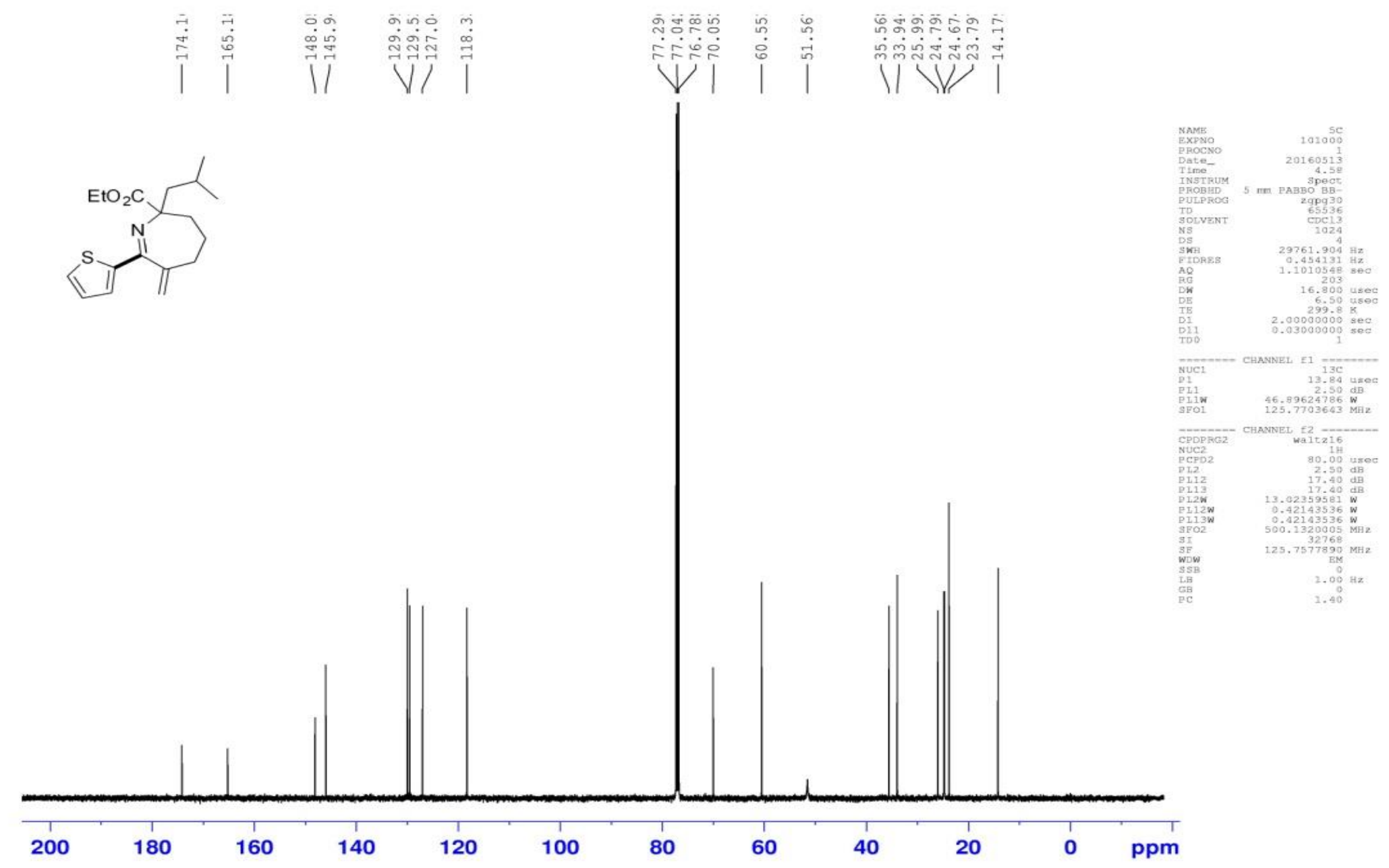




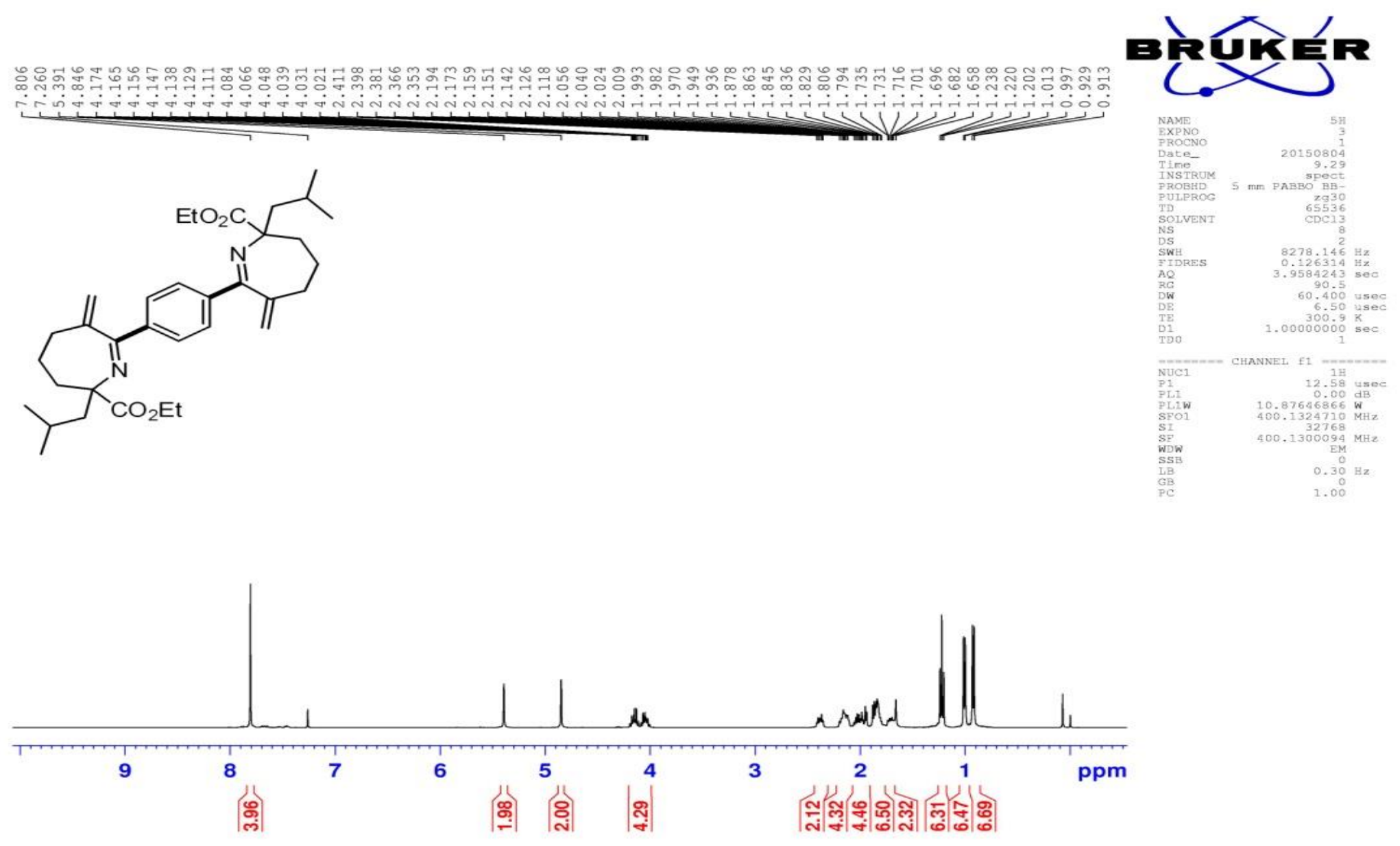




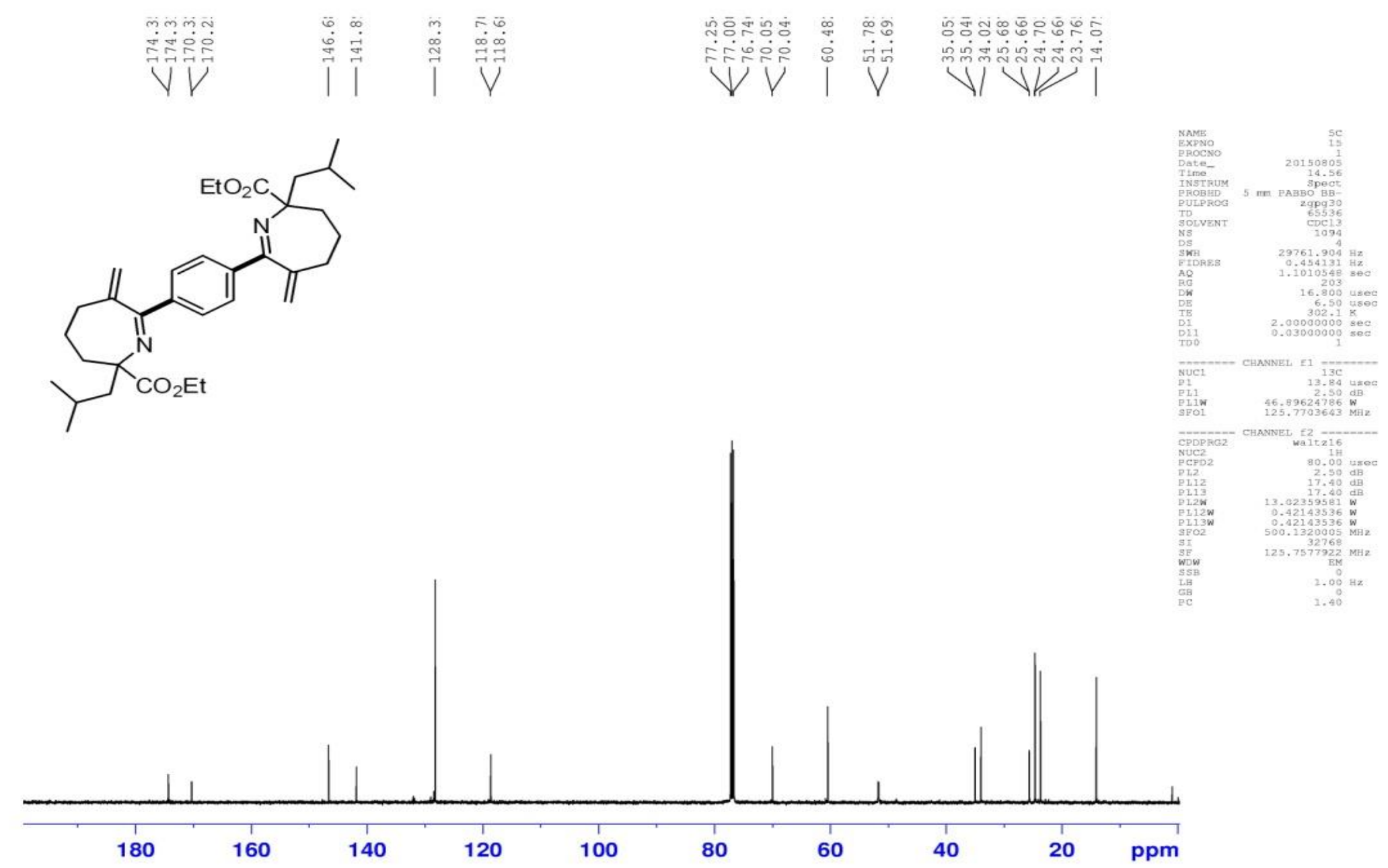




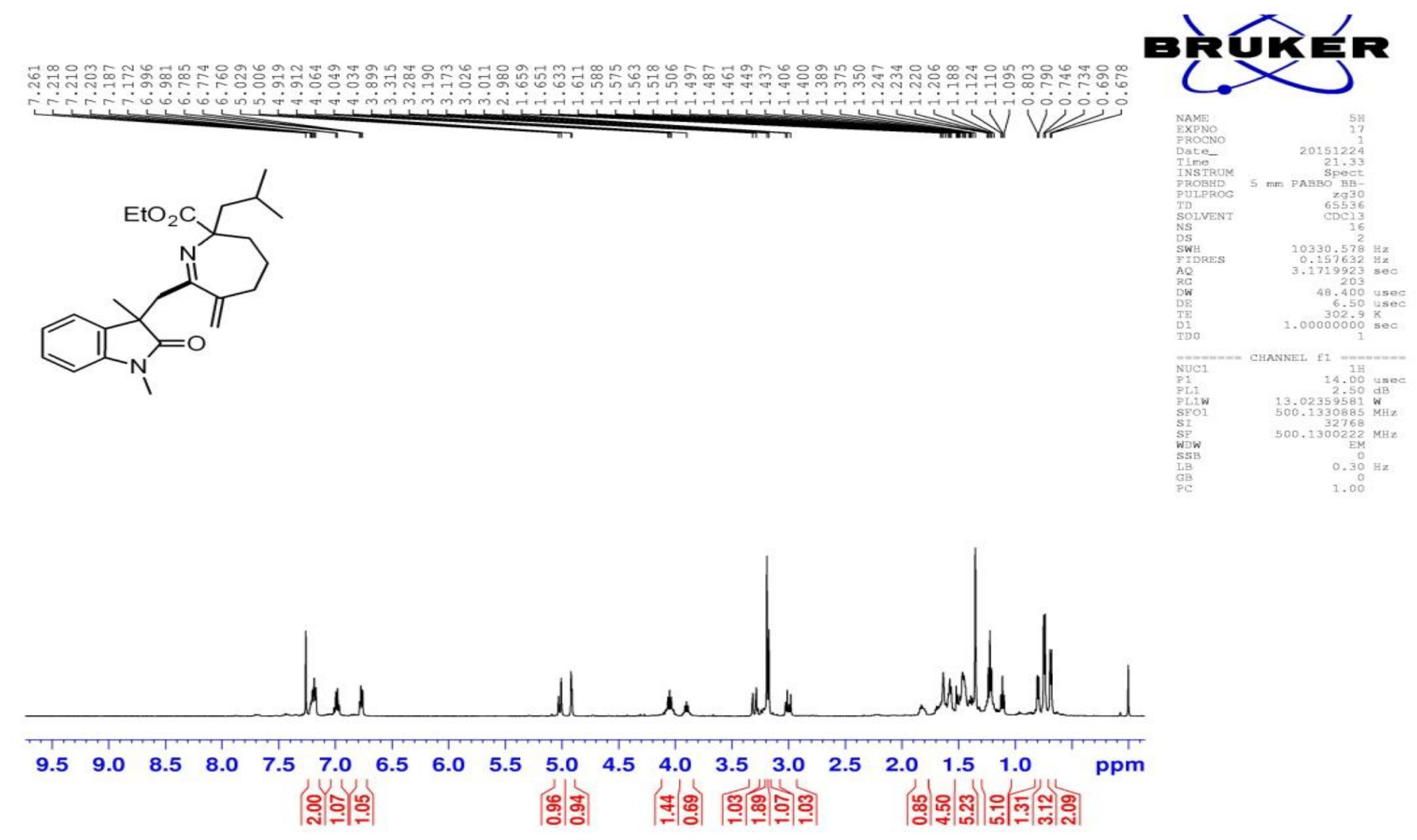




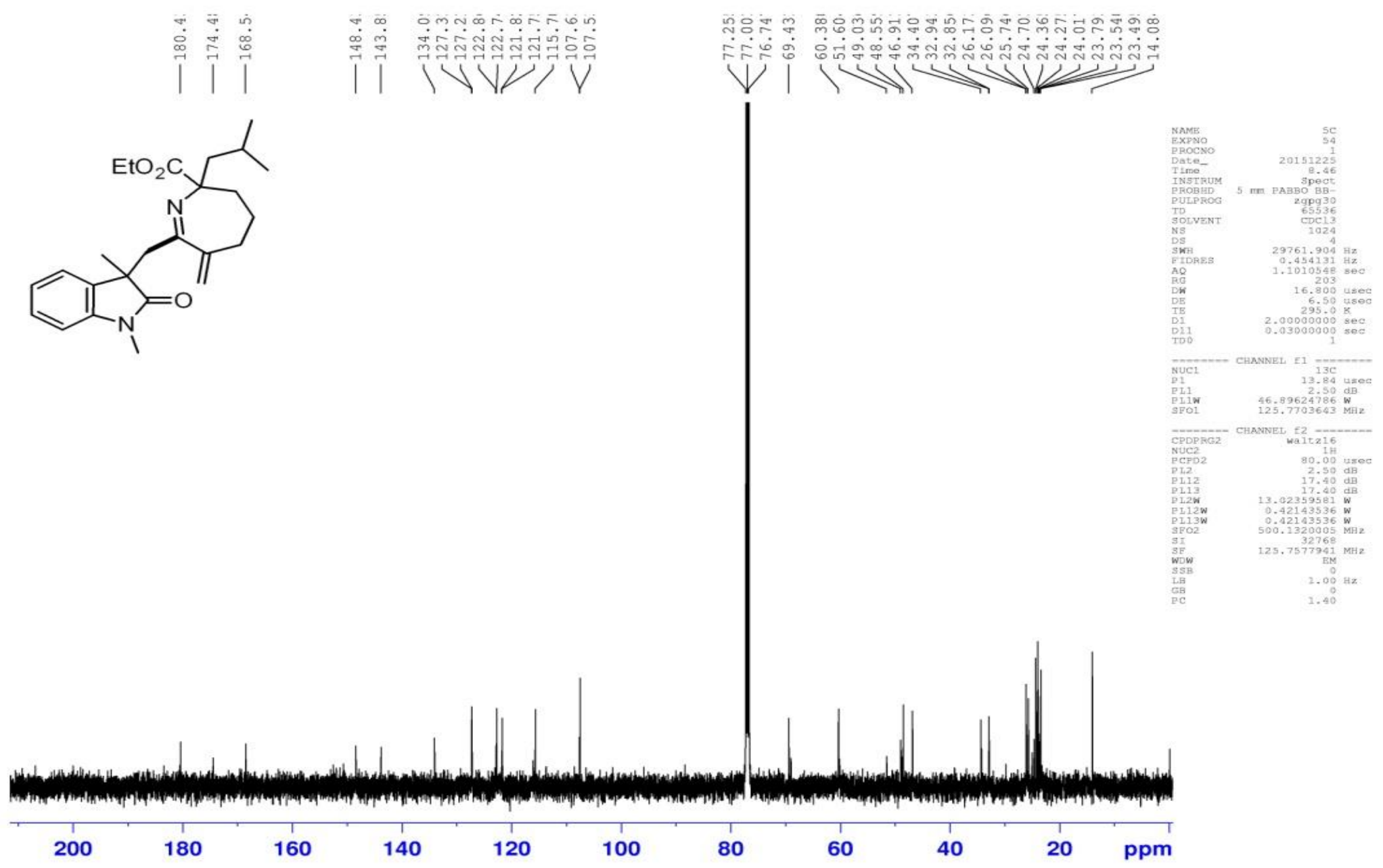




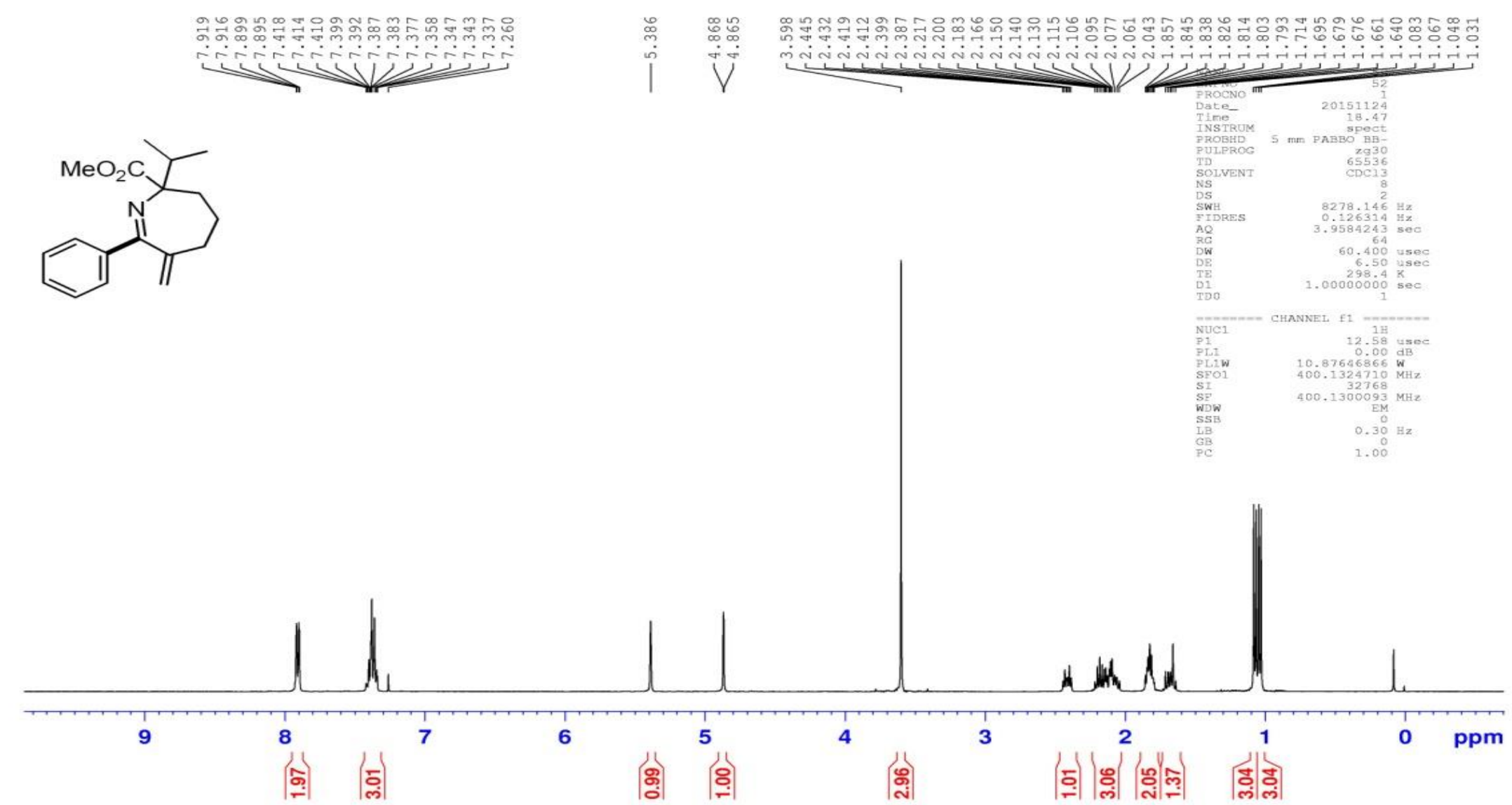




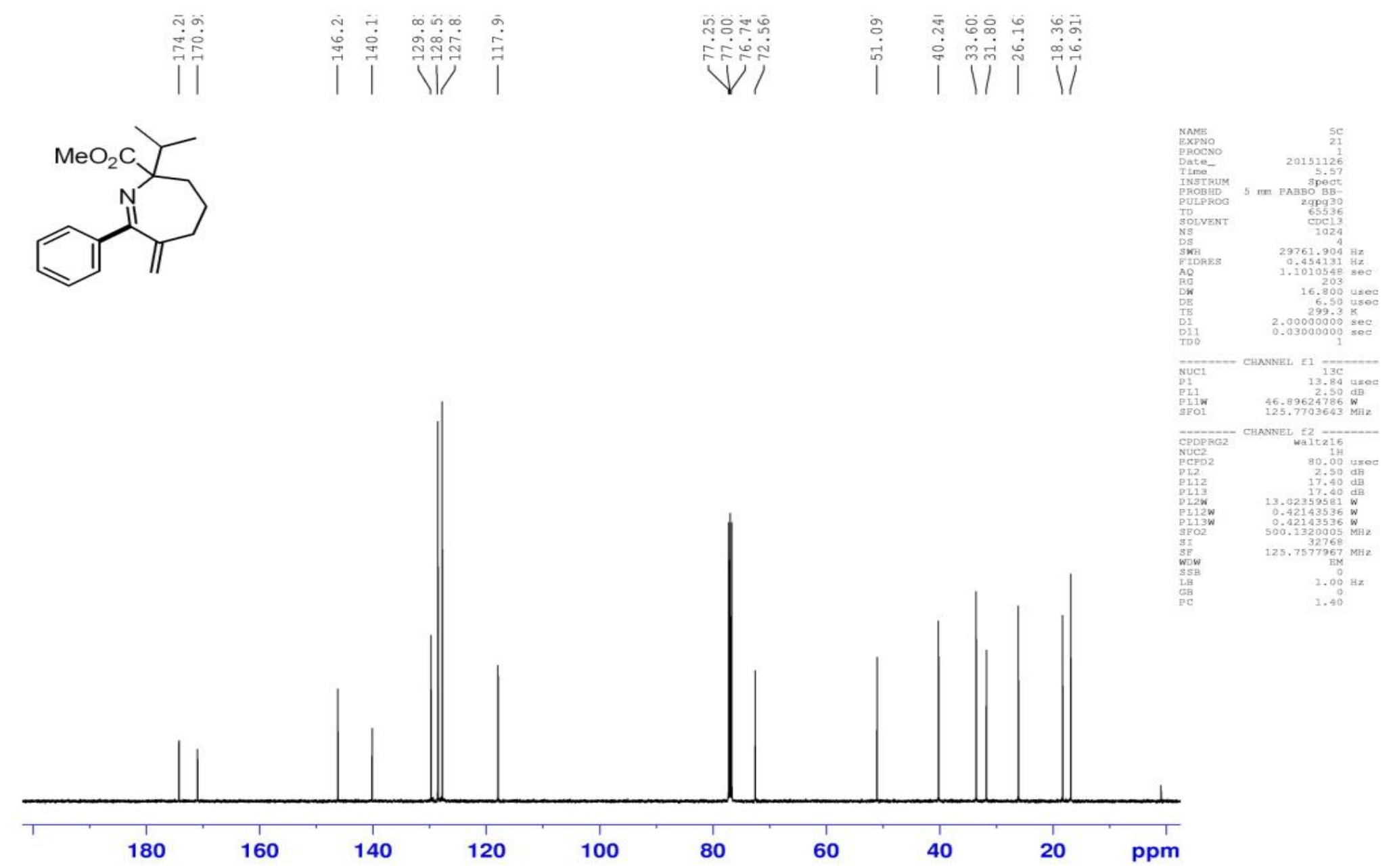




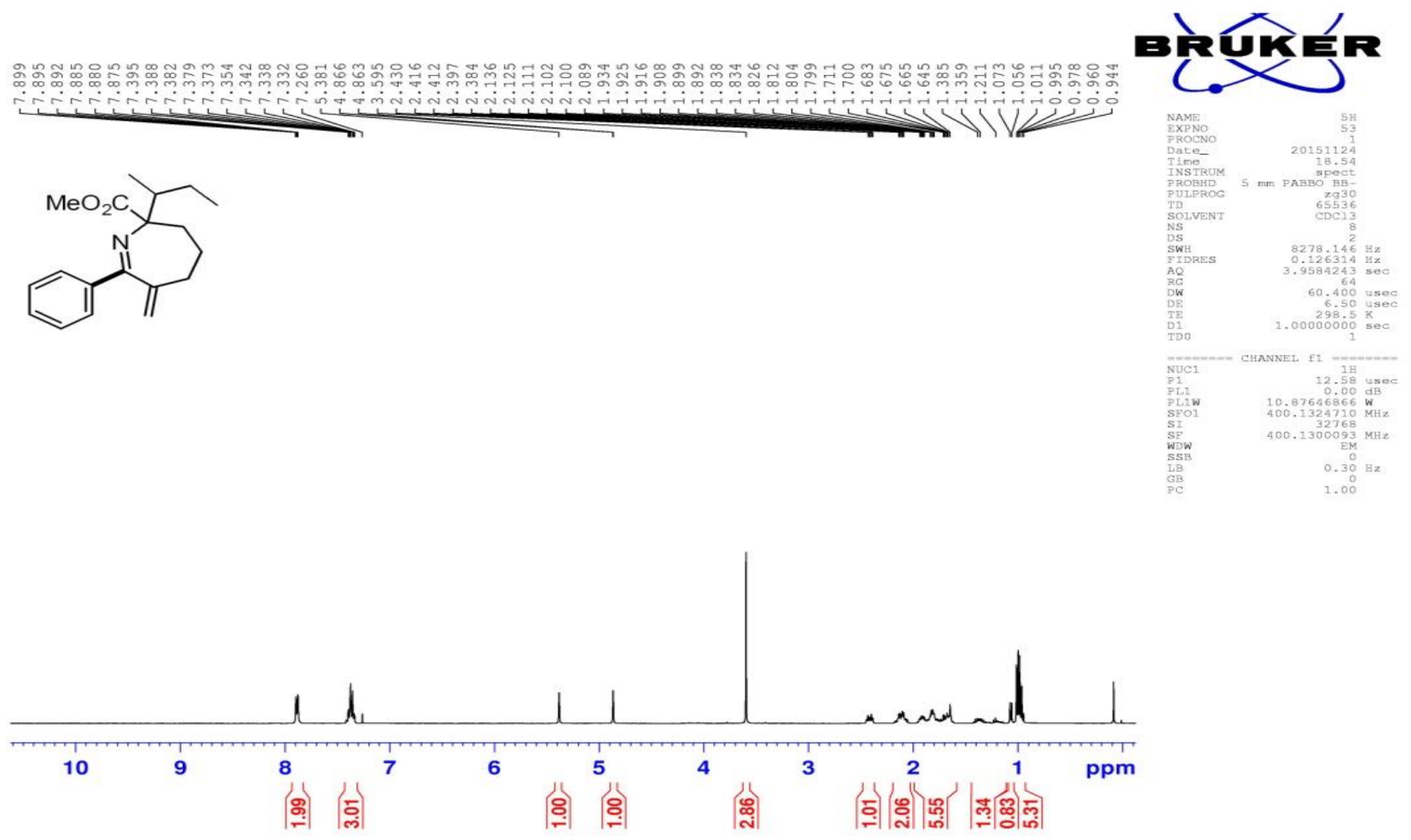




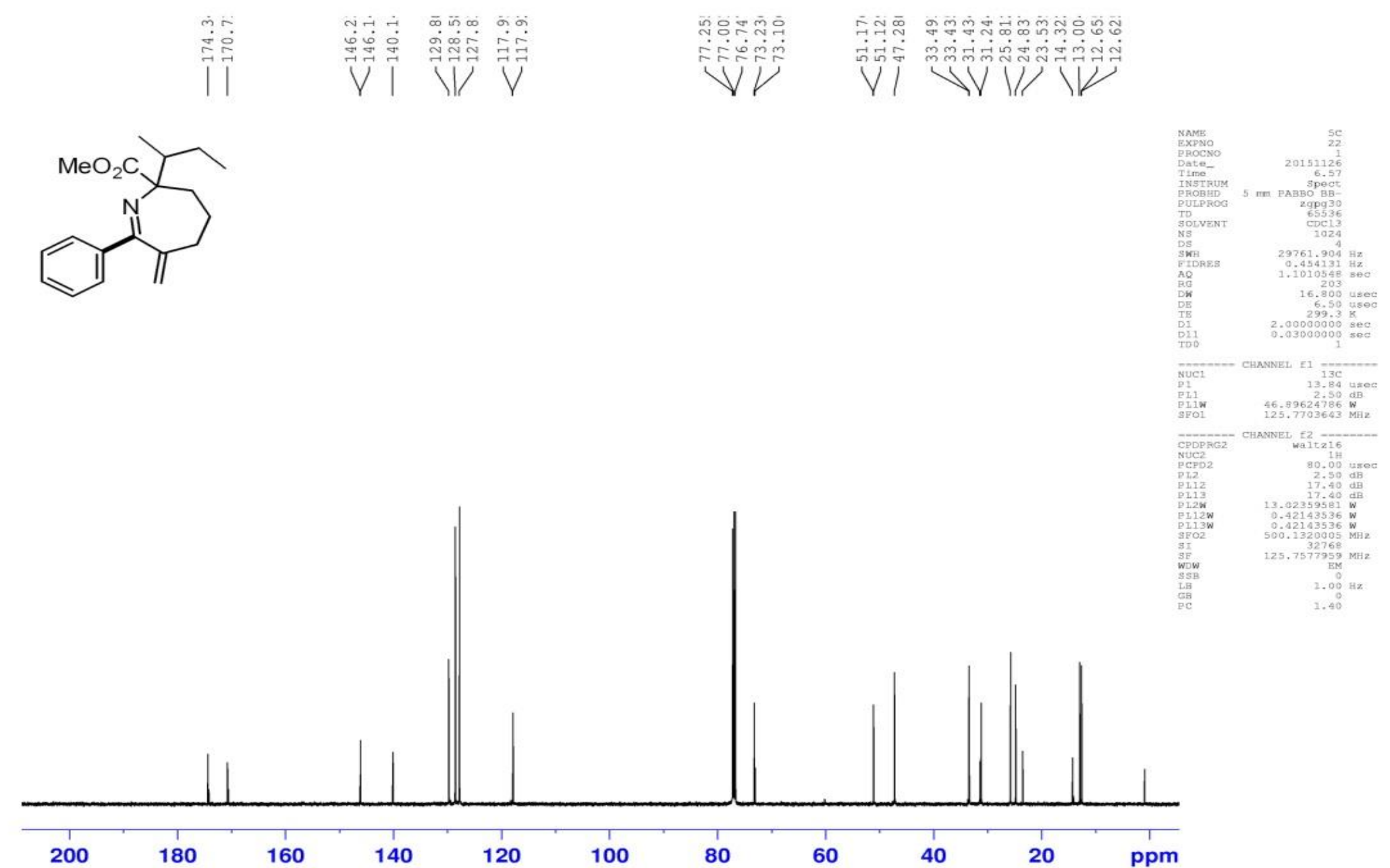




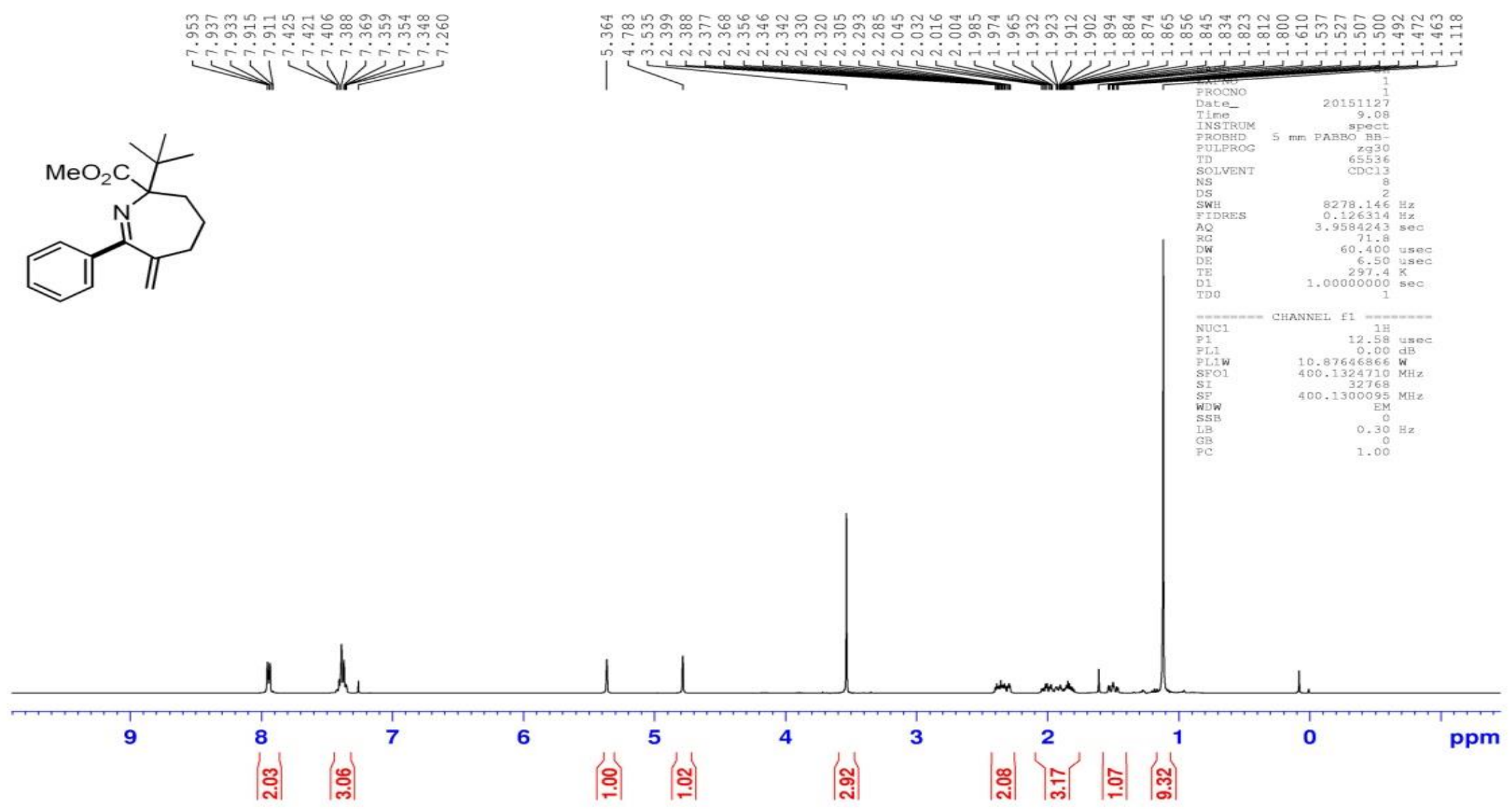




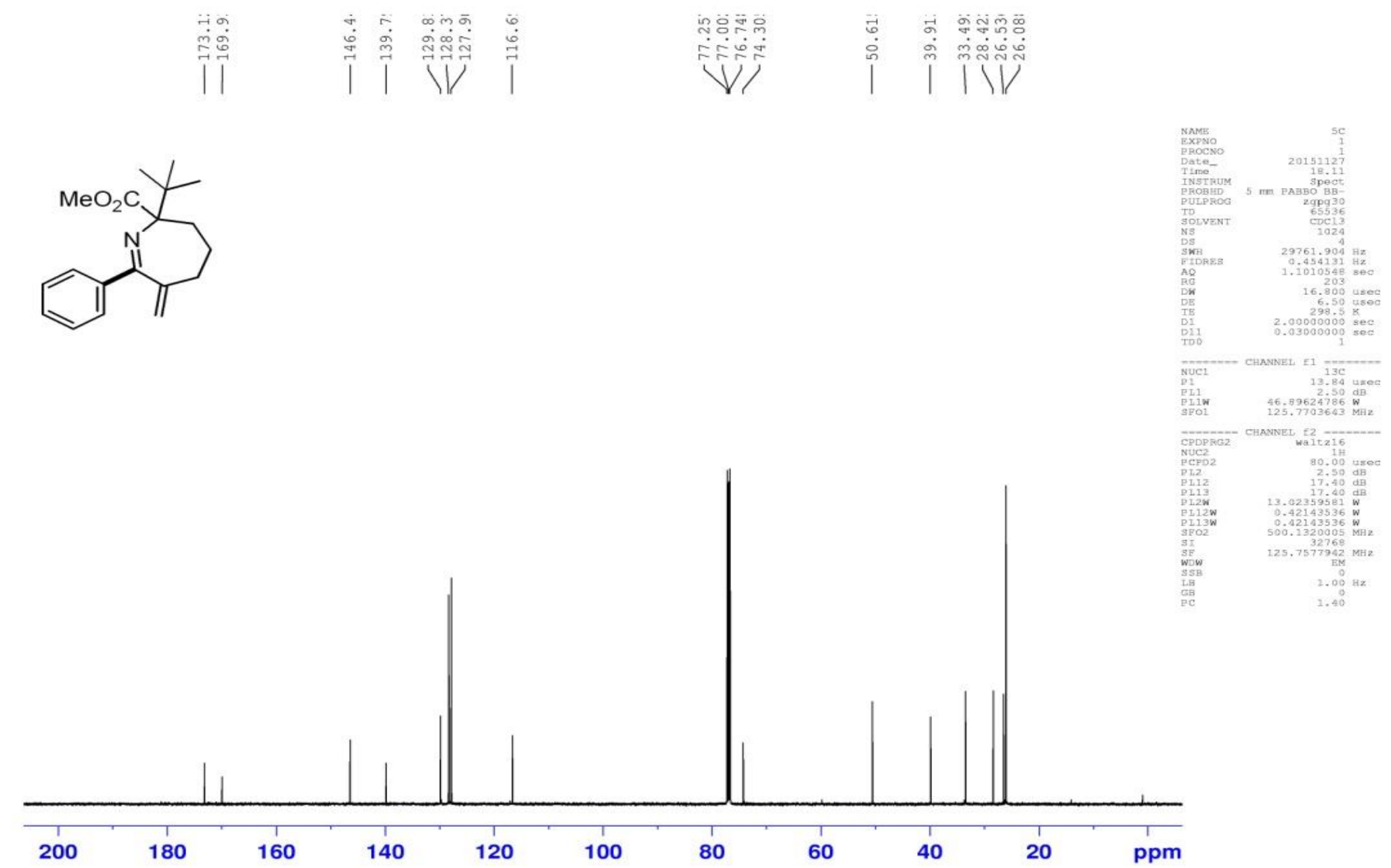




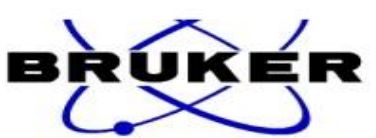

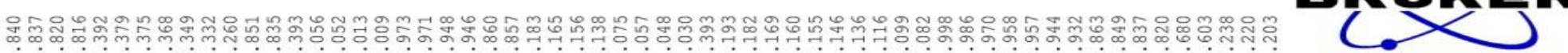
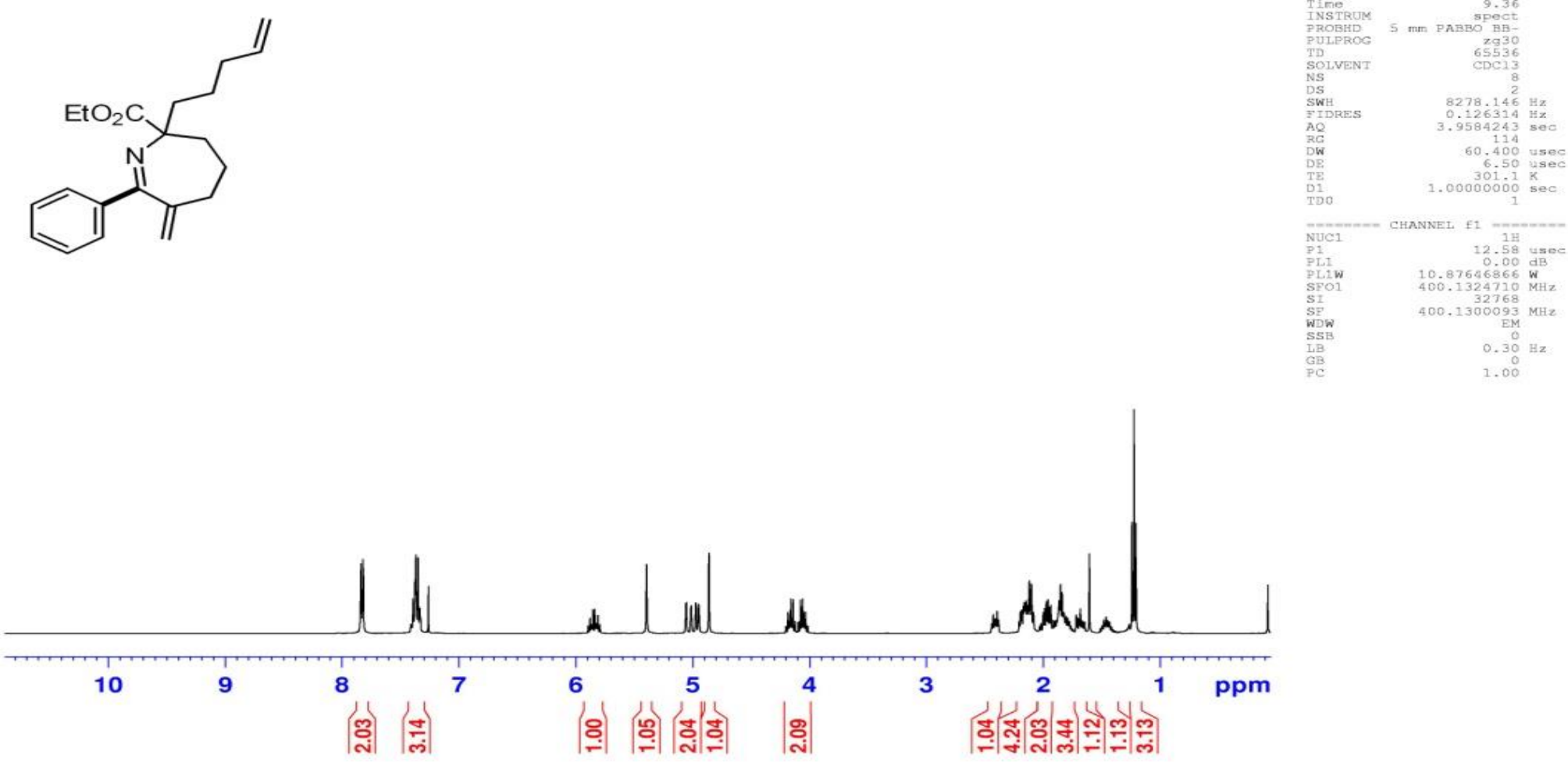


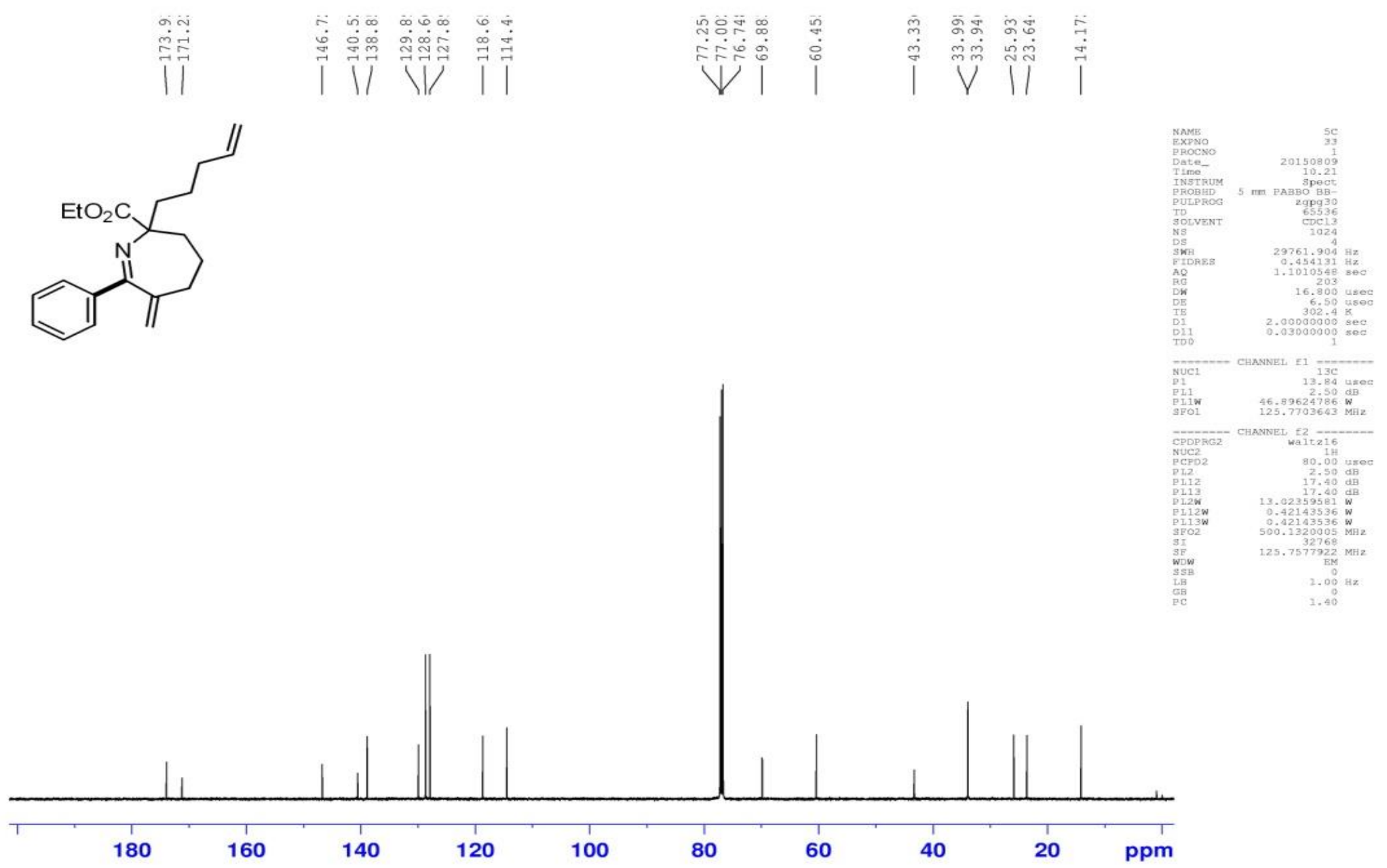




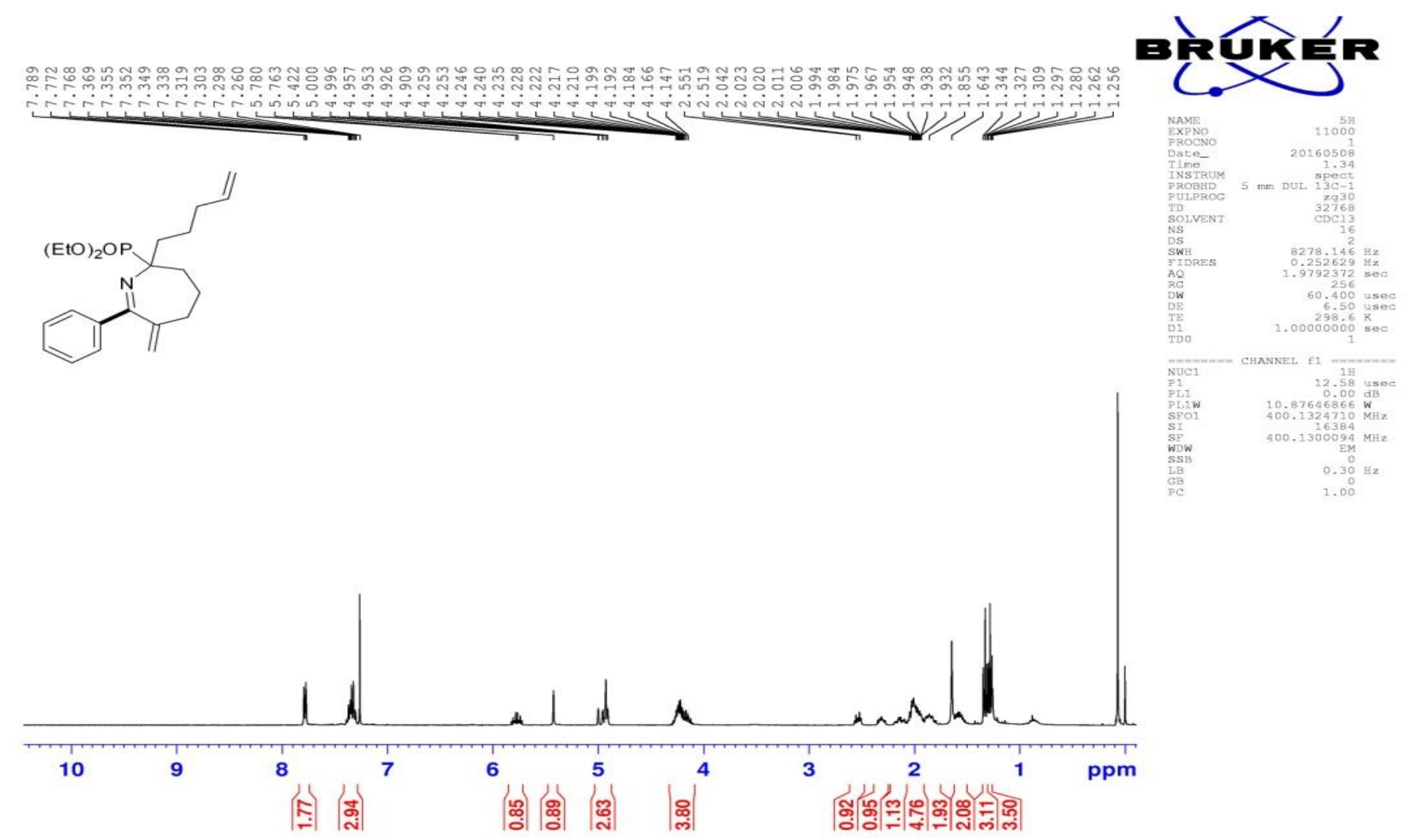




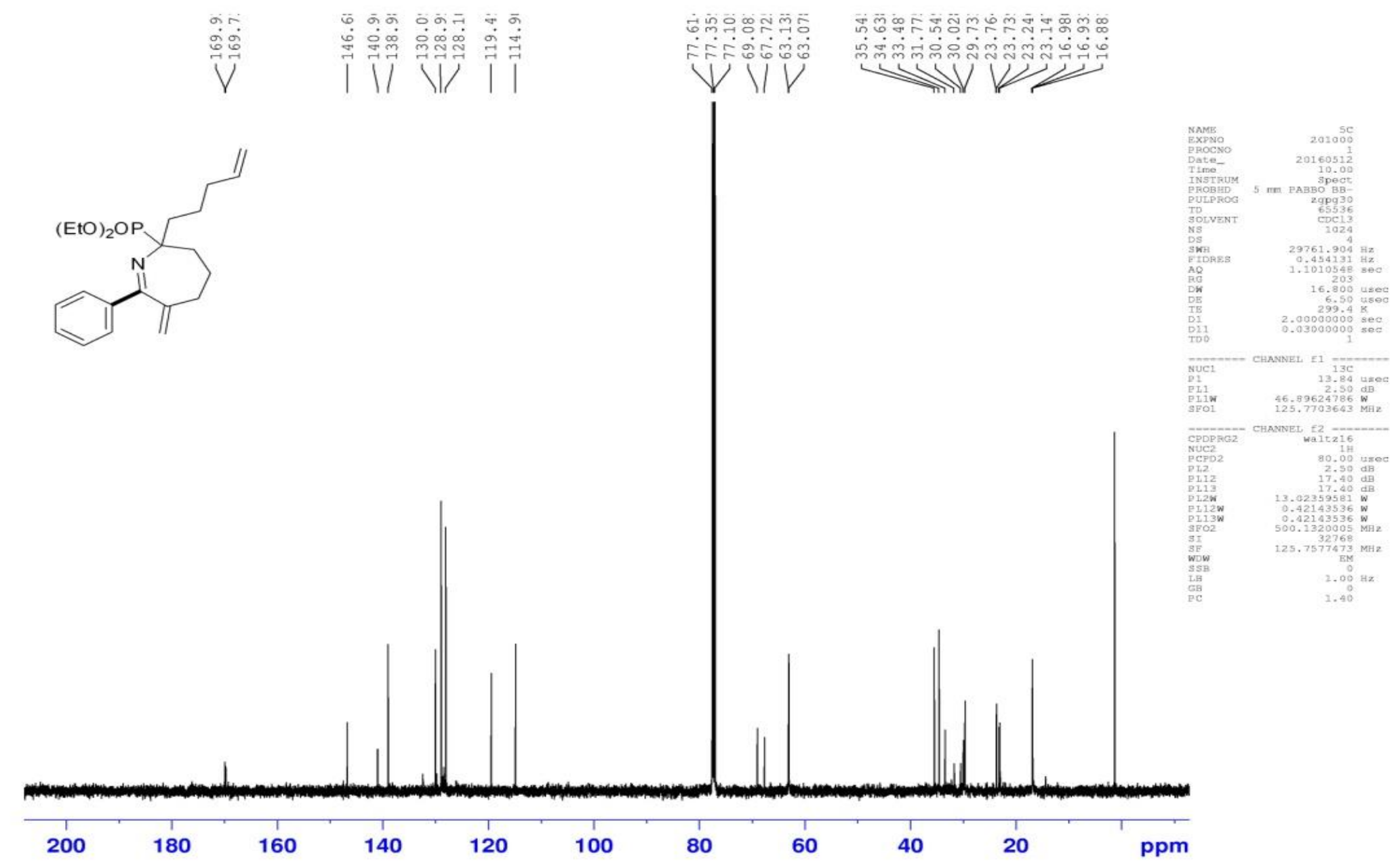




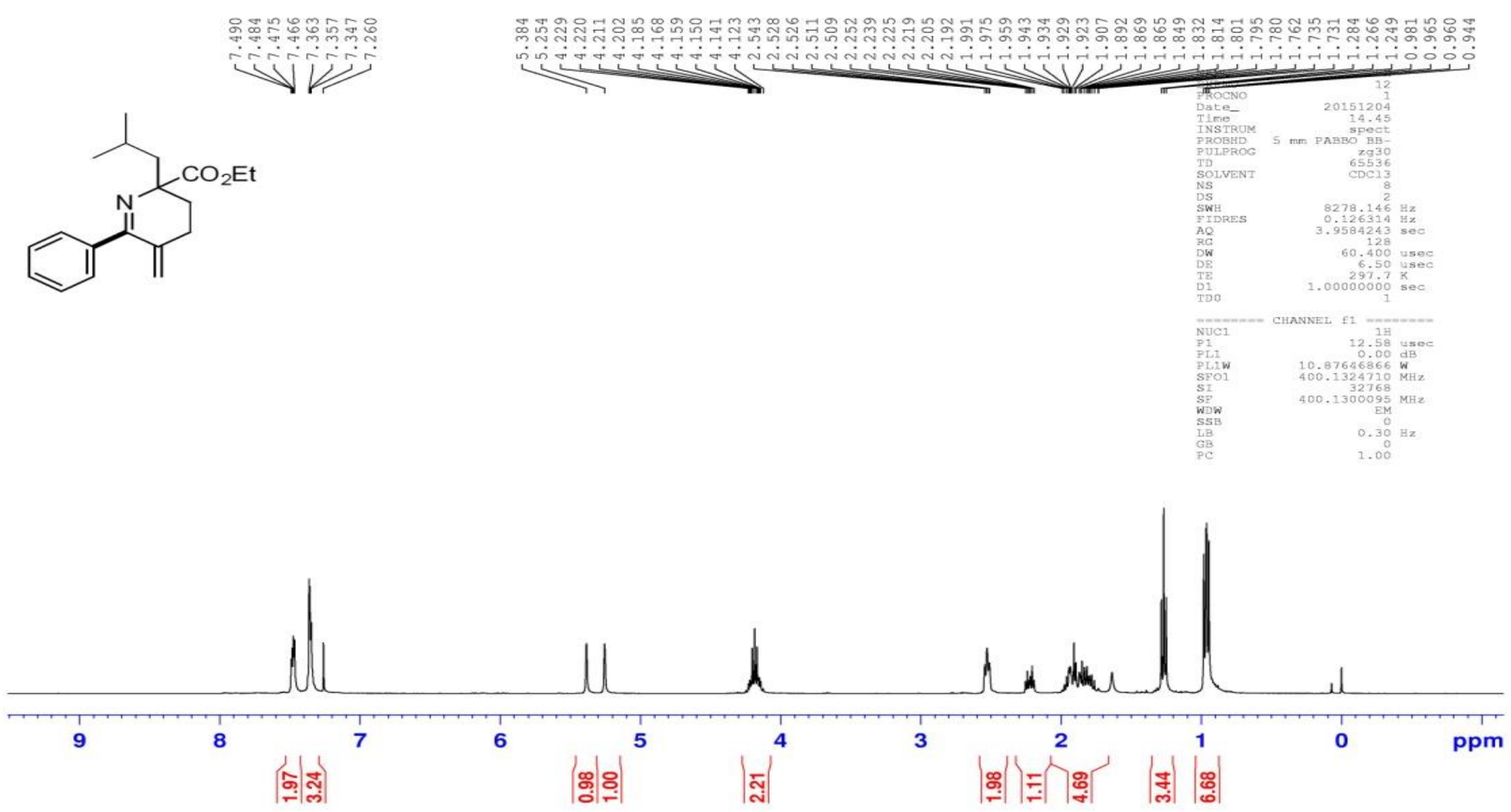




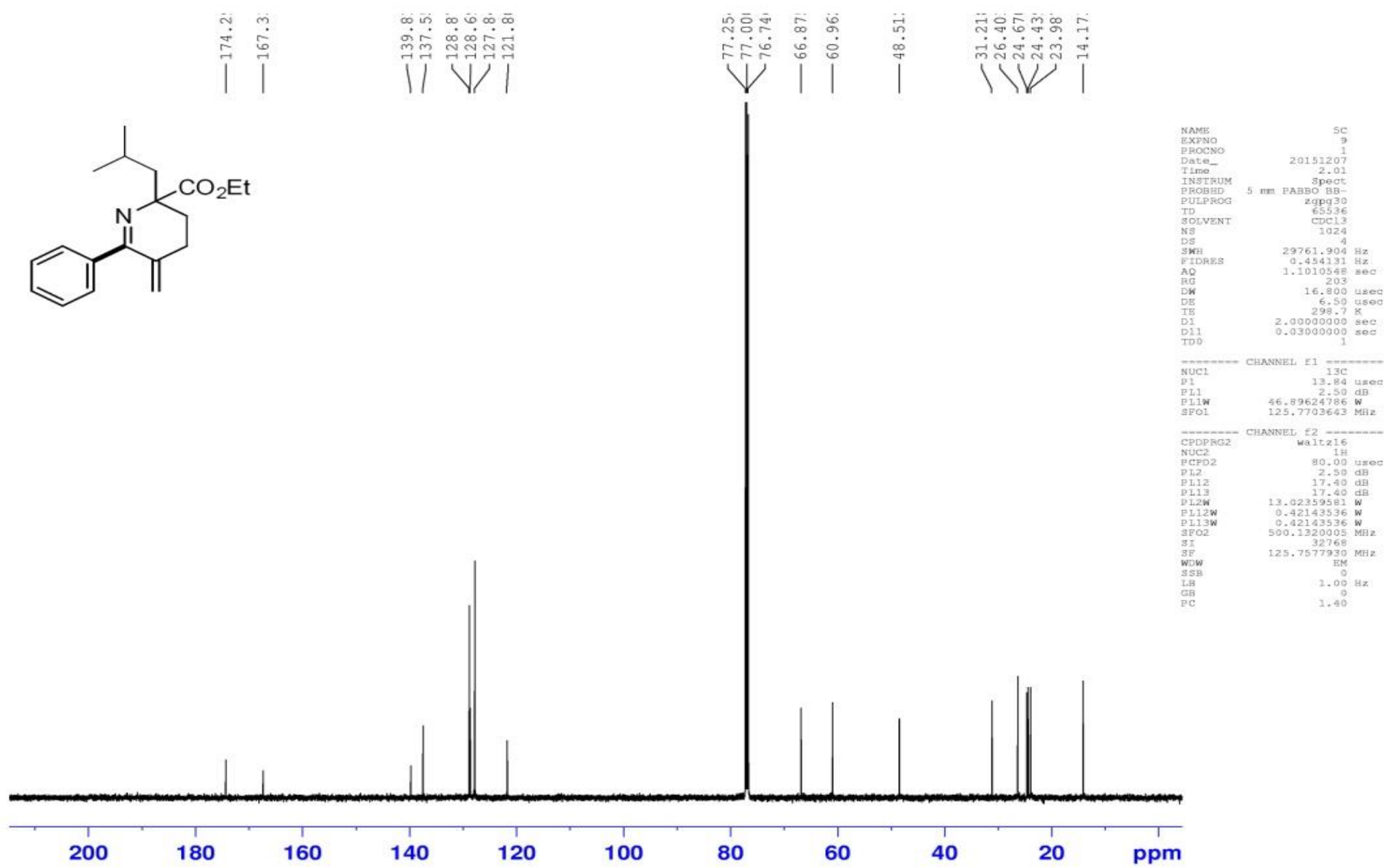

102 


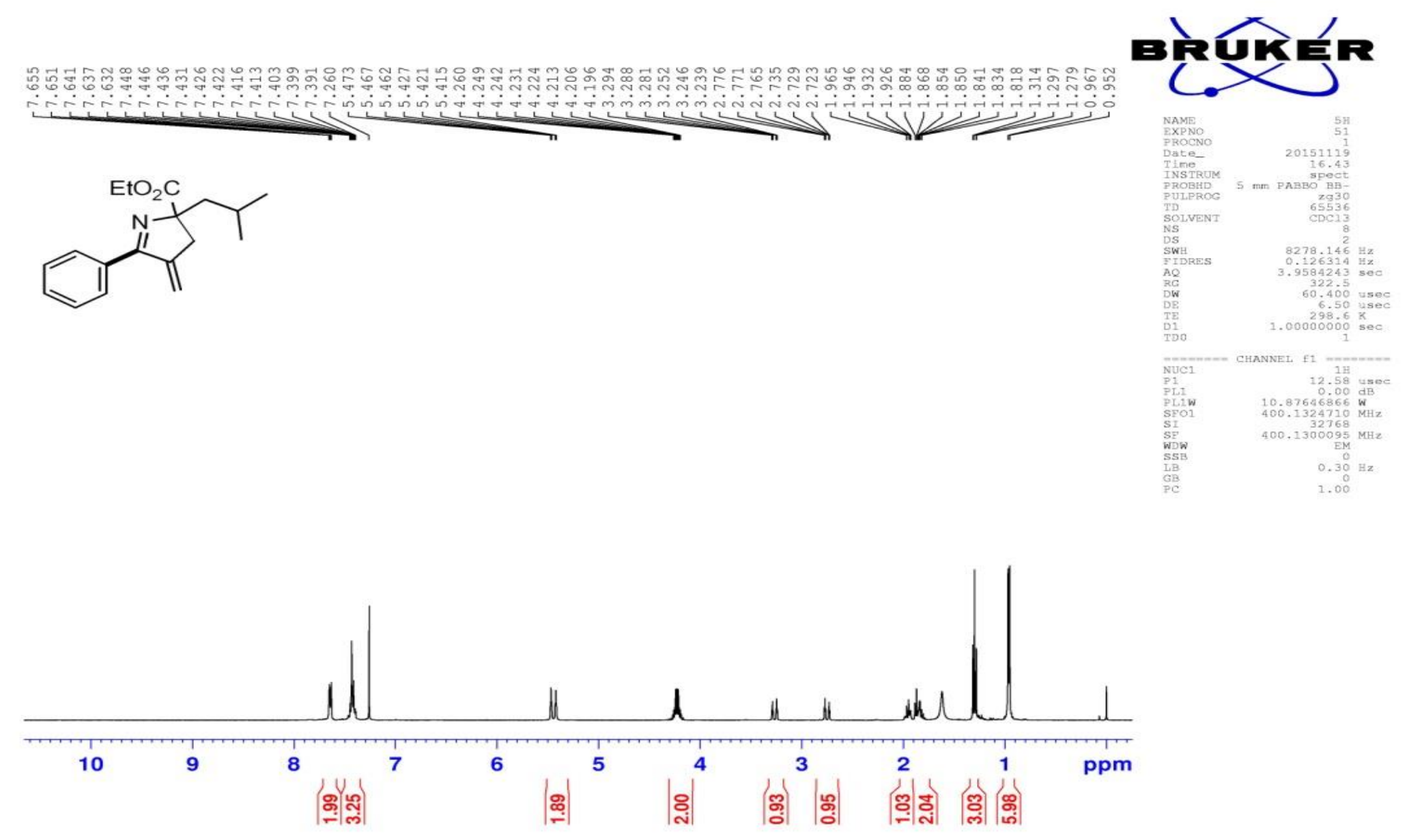




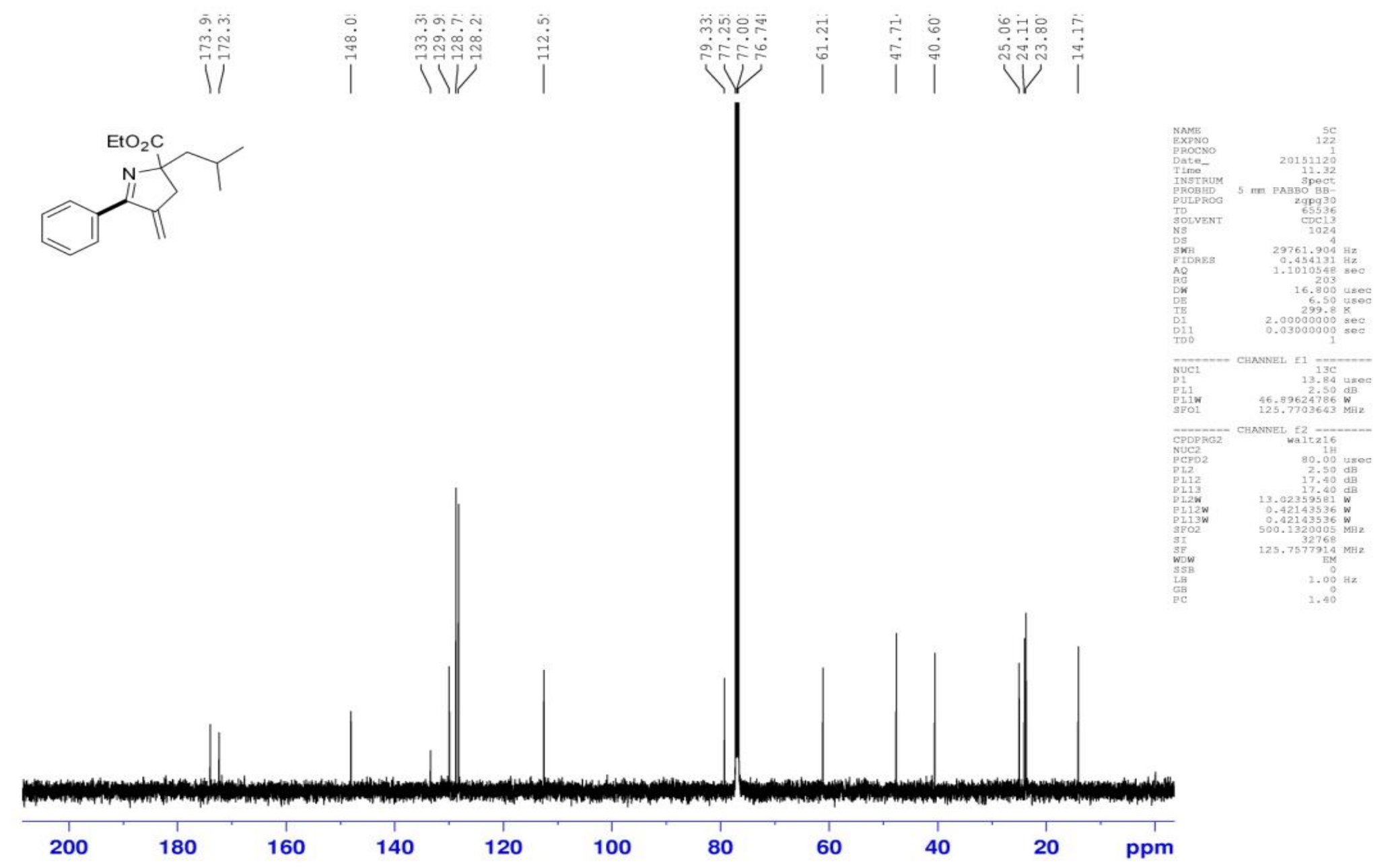

104 


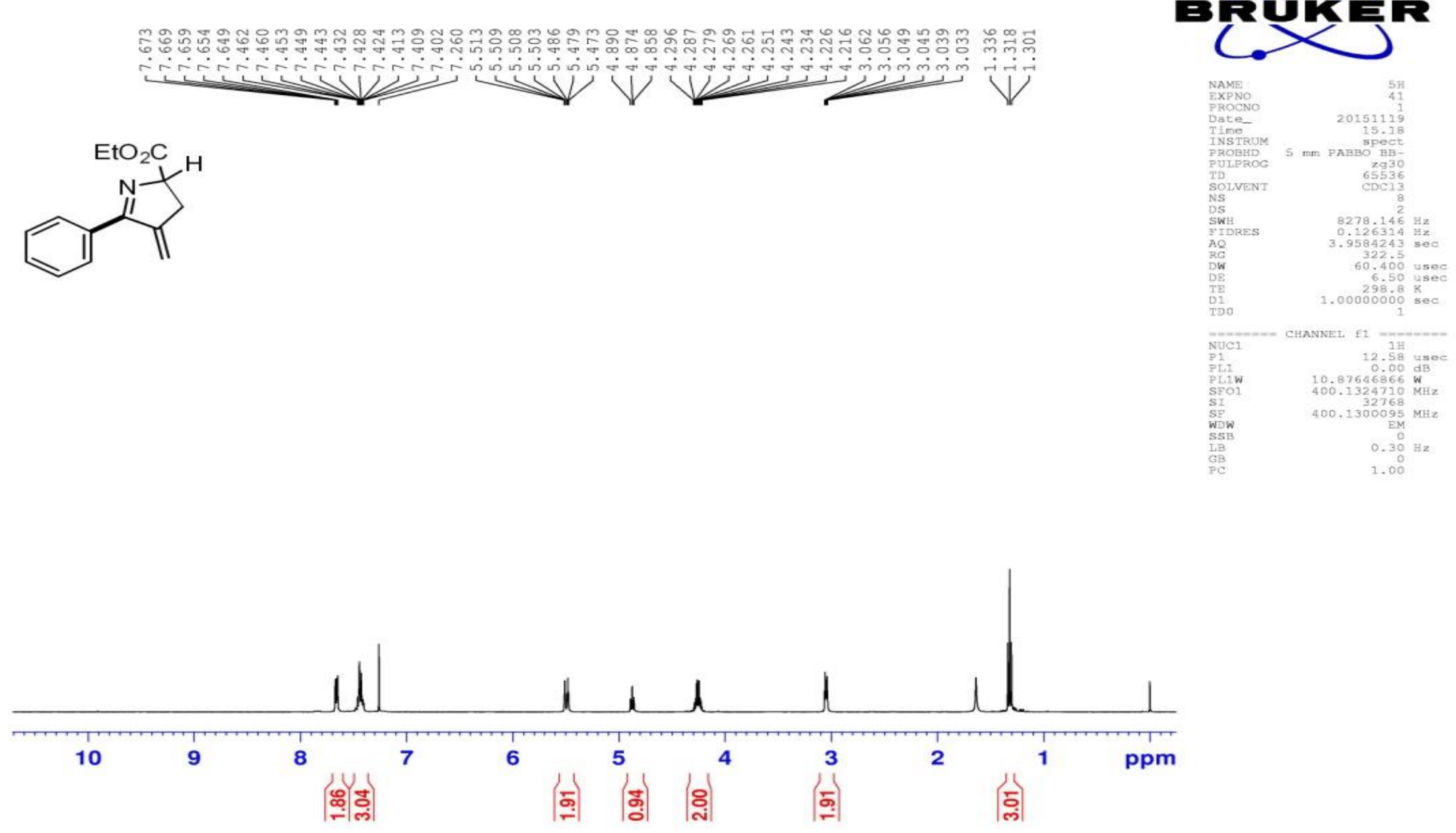




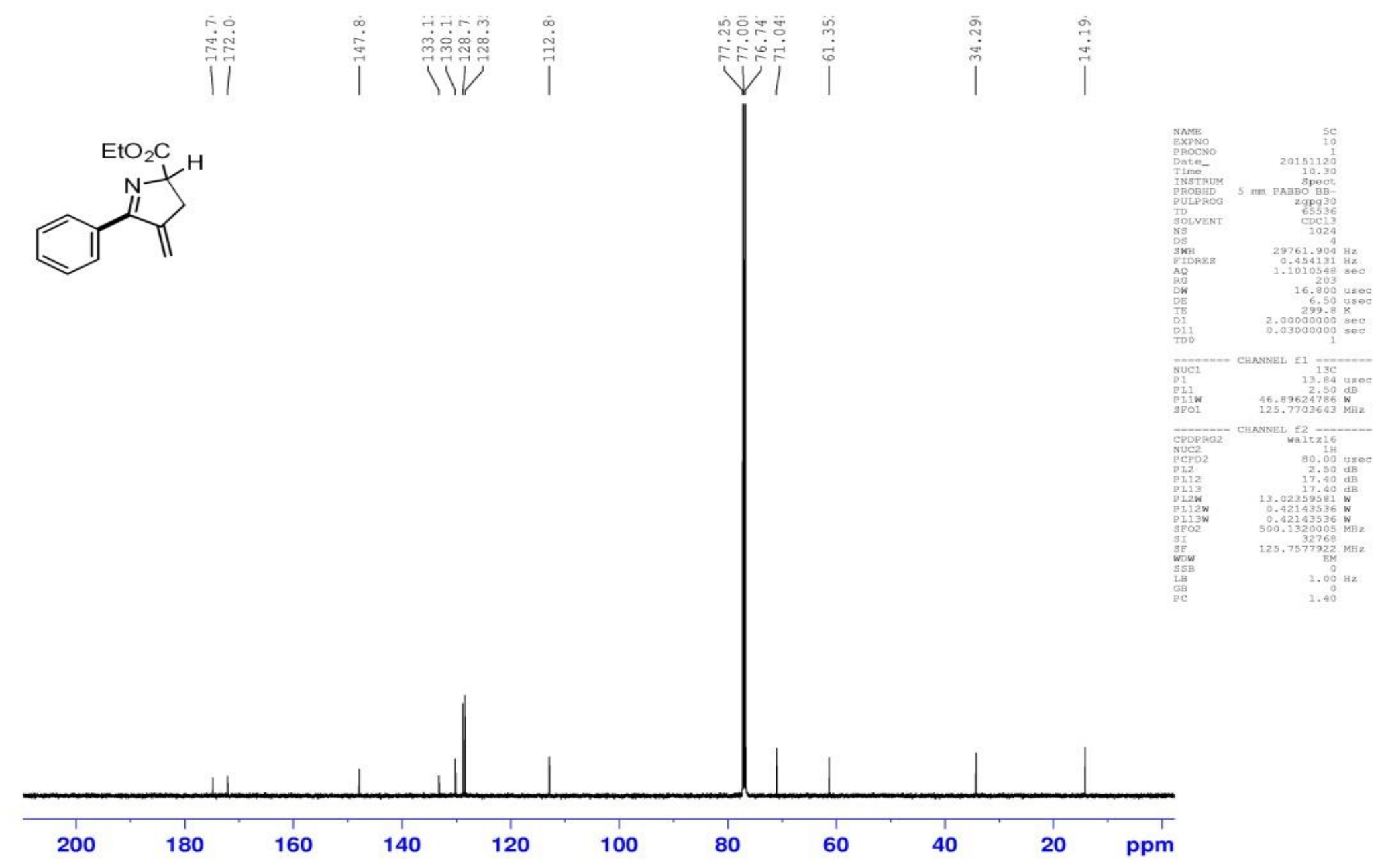




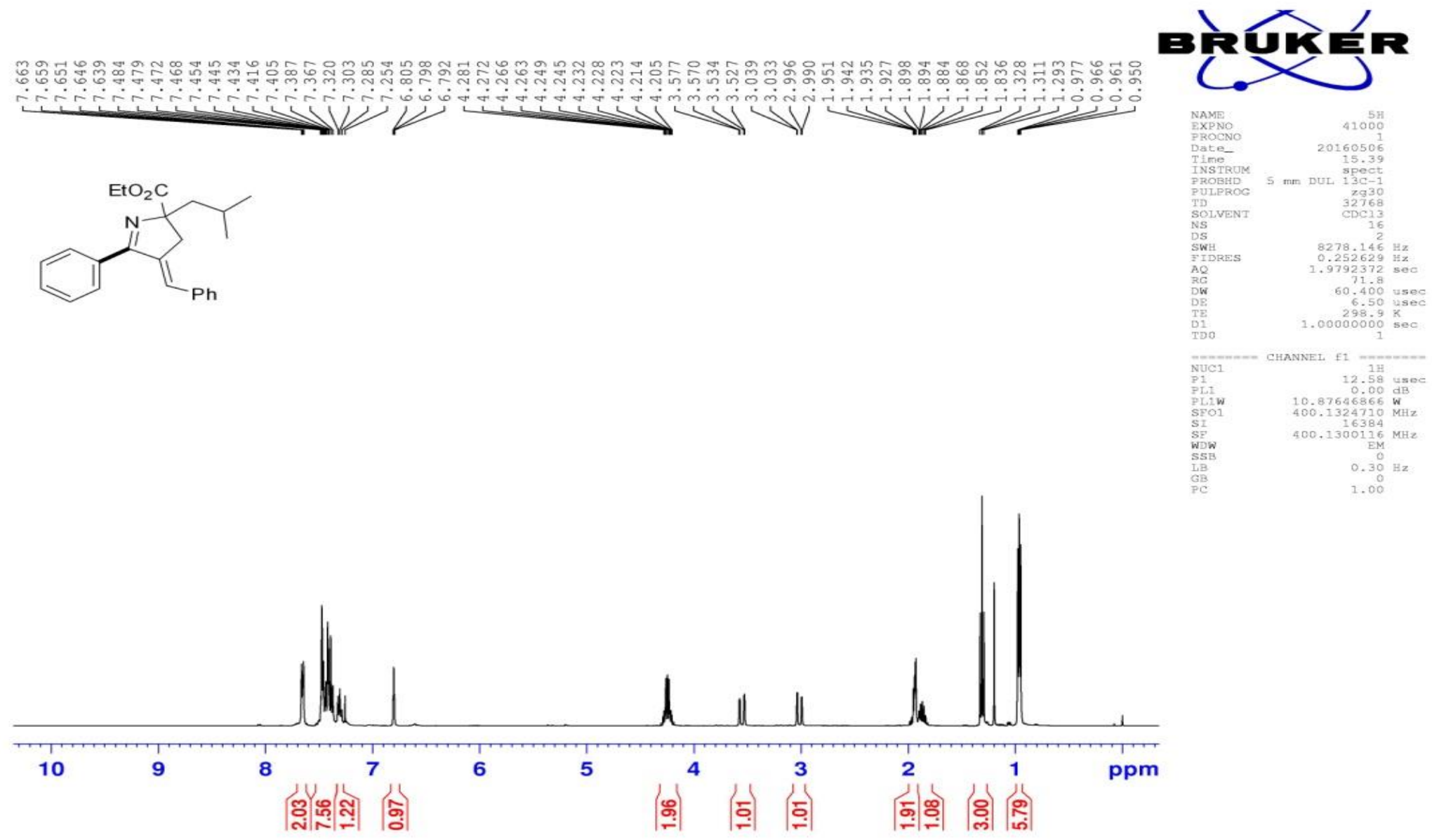




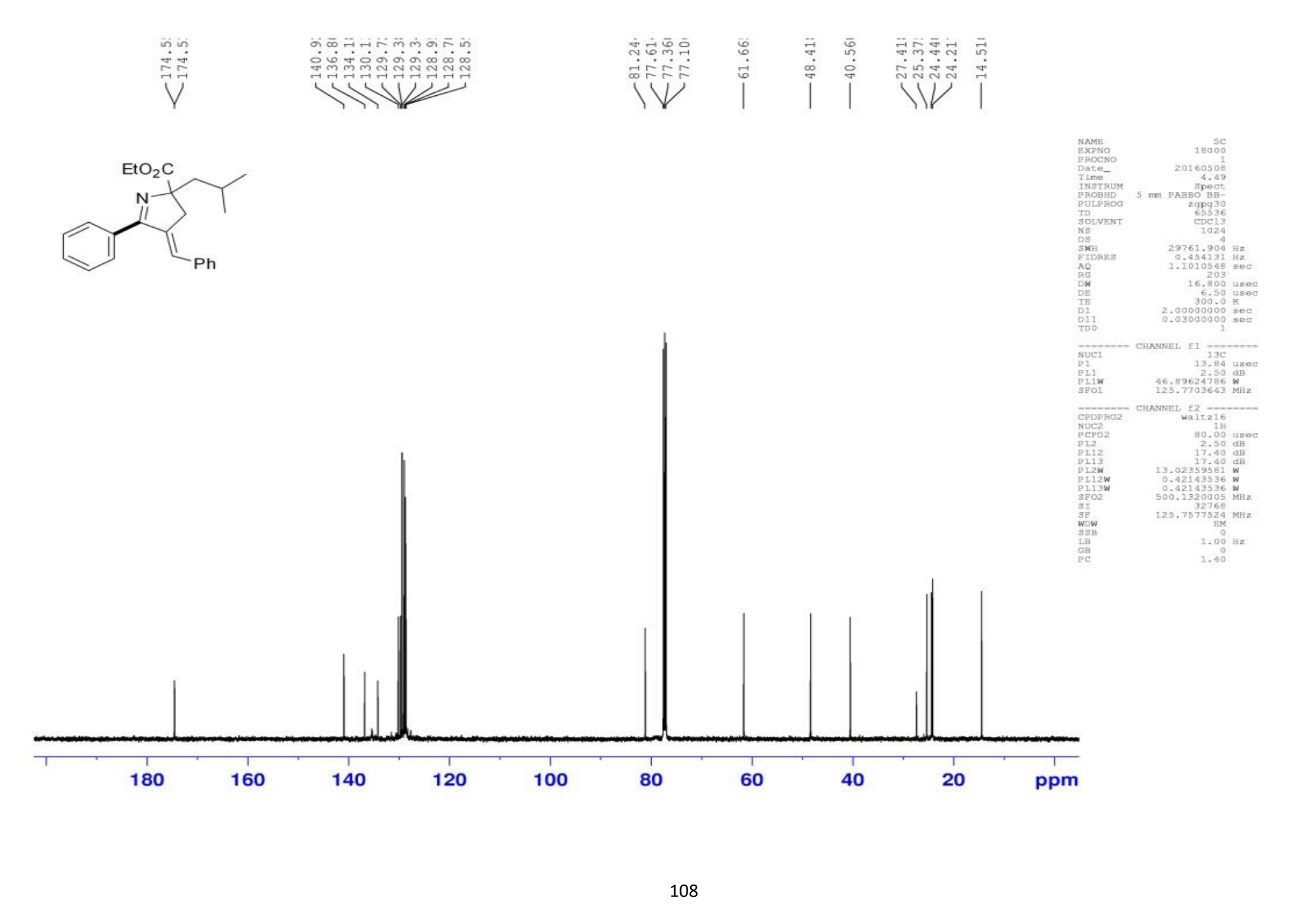




\section{4i-HMBC}

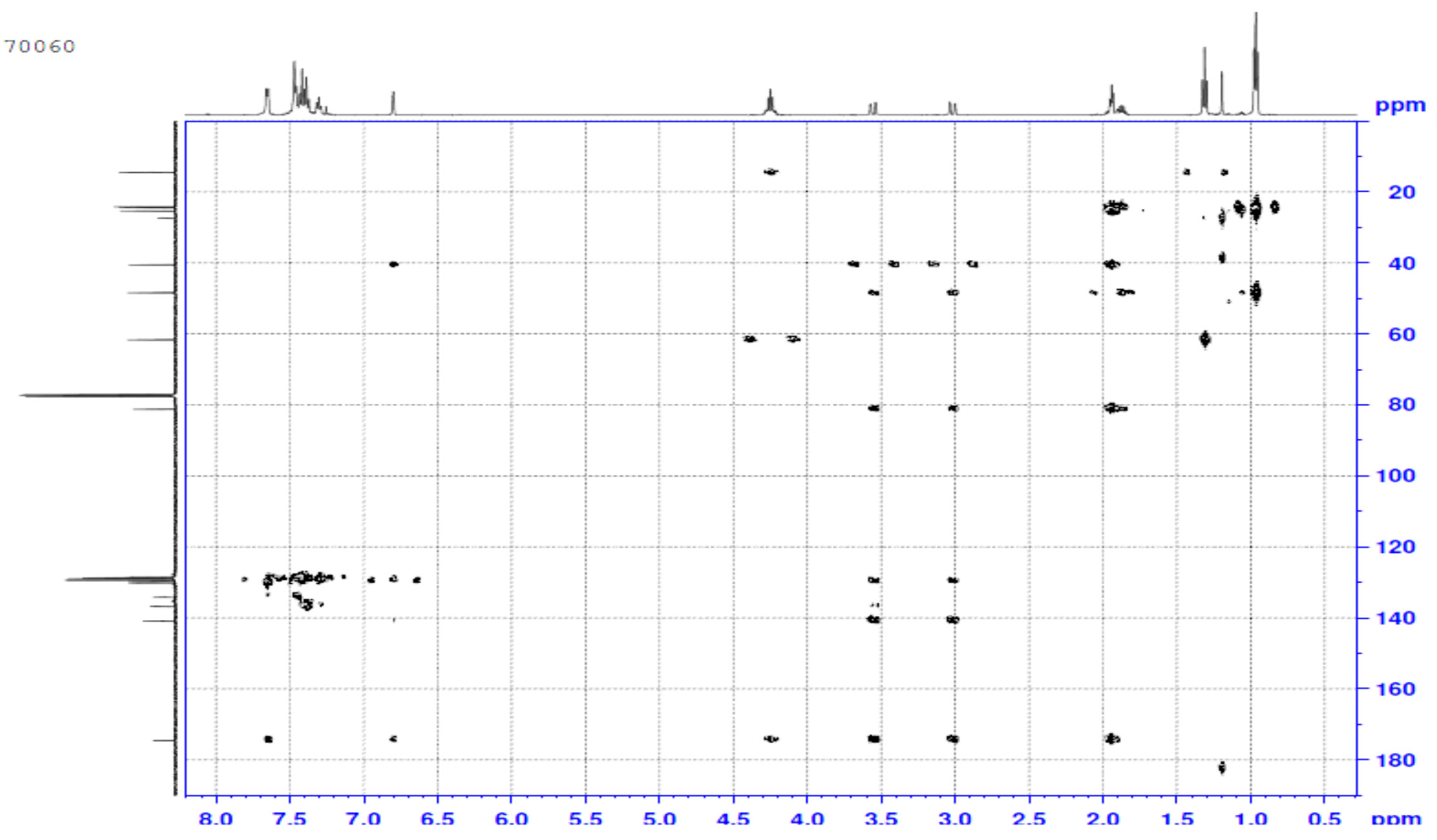


4i-NOE

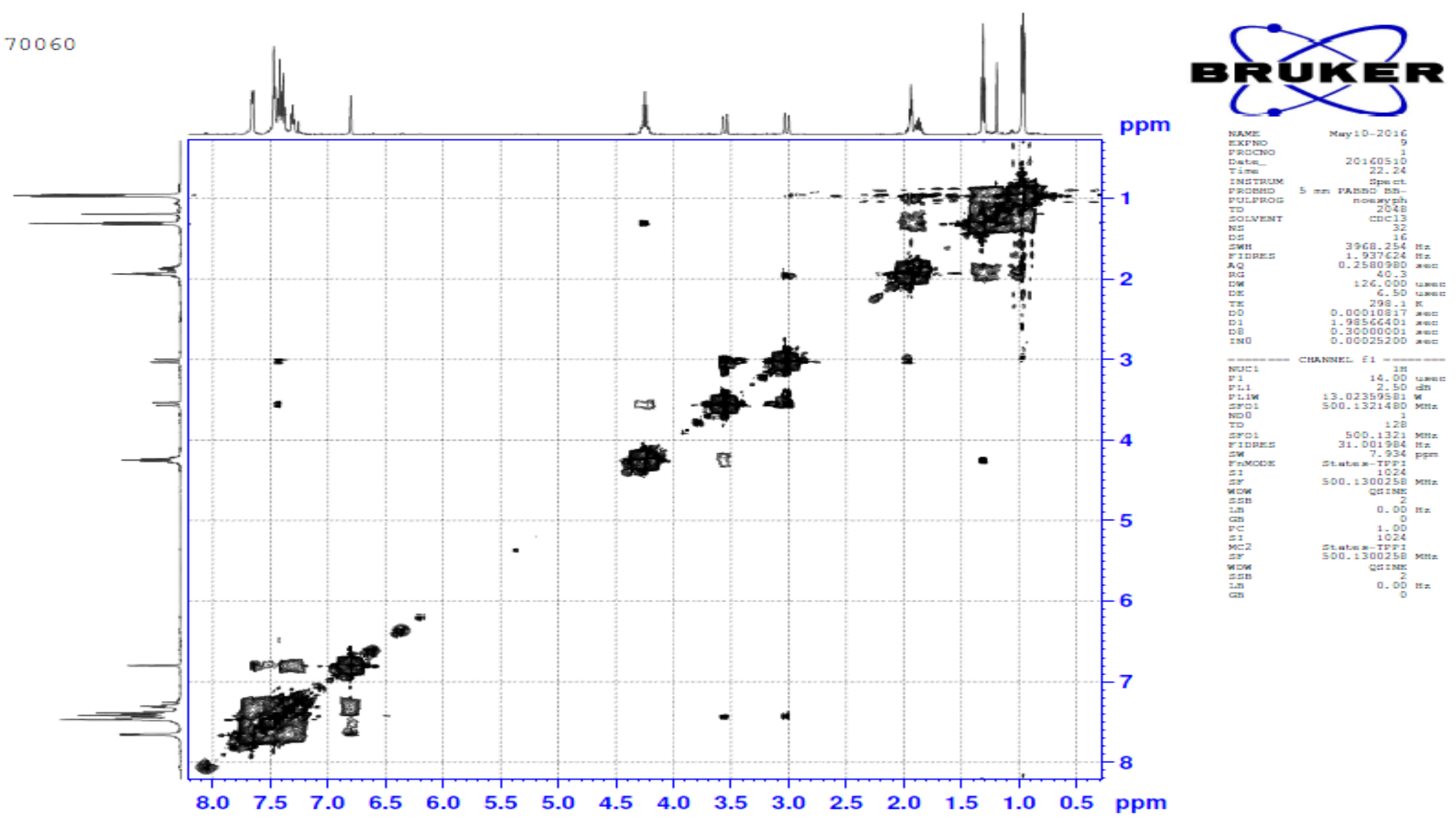

